University of Louisville

ThinkIR: The University of Louisville's Institutional Repository

Electronic Theses and Dissertations

$5-2018$

\title{
PLGA-modified nanoparticles for the treatment of hypo- vascularized HPV-related cervical cancers.
}

Lee B. Sims

University of Louisville

Follow this and additional works at: https://ir.library.louisville.edu/etd

Part of the Biomaterials Commons, and the Molecular, Cellular, and Tissue Engineering Commons

\section{Recommended Citation}

Sims, Lee B., "PLGA-modified nanoparticles for the treatment of hypo-vascularized HPV-related cervical cancers." (2018). Electronic Theses and Dissertations. Paper 2892.

https://doi.org/10.18297/etd/2892

This Master's Thesis is brought to you for free and open access by ThinkIR: The University of Louisville's Institutional Repository. It has been accepted for inclusion in Electronic Theses and Dissertations by an authorized administrator of ThinkIR: The University of Louisville's Institutional Repository. This title appears here courtesy of the author, who has retained all other copyrights. For more information, please contact thinkir@louisville.edu. 
PLGA-modified Nanoparticles for the Treatment of Hypo-Vascularized HPV-related Cervical Cancers

By

Lee B. Sims B.S., University of Louisville, 2017

\author{
A Thesis \\ Submitted to the Faculty of the \\ University of Louisville \\ J. B. Speed School of Engineering \\ as Partial Fulfillment of the Requirements \\ for the Professional Degree
}

\title{
MASTER OF ENGINEERING
}

Department of Bioengineering

April 2018 
PLGA-modified Nanoparticles for the Treatment of Hypo-Vascularized HPV-related Cervical Cancers

Submitted by:

Lee B. Sims

A Thesis Approved On

Date

By the Following Reading and Examination Committee

$\overline{\text { Dr. Jill Steinbach-Rankins, Thesis Director }}$

Dr. Hermann Frieboes

Dr. David Hein 


\section{ACKNOWLEDGMENTS}

Words cannot express the thanks and gratitude I feel for the mentorship Dr. Jill Steinbach-Rankins has given me over the past five years. Working as a student in her lab throughout my undergraduate and graduate career has provided me with opportunities I would not have imagined at the beginning of my collegiate career. Dr. Steinbach-Rankins always went above and beyond her duty as an academic mentor, encouraging me to pursue my intellectual passions and seek out opportunities that would help me grow both professionally and personally. She fosters a lab culture that is encouraging, motivating, and enriching as well as genuinely taking all of her student's best interests to heart. In reflecting over the past five years, the mentorship Dr. Steinbach-Rankins provided has tremendously impacted me as a scientist and has helped inform and mold my academic and professional trajectory. She has set an exceptional precedent as a mentor and was always eager and willing to help me in pursuing my goals and ambitions. I am beyond honored to be able to formally recognize and give thanks for all that she has done for me.

I would also like to thank Dr. Hermann Frieboes for his additional mentorship with my research experience here at UofL. Dr. Frieboes has provided me with many skills that I will be able to draw upon no matter where my career takes me. He was always passionate about the work we were doing, and made sure that I was maximizing my research opportunities to reach my full potential as a student and scientist. The accomplishments I have had at UofL and the opportunities that lie before me would not be possible without his mentorship.

Additionally, I would like to thank all of my peers, colleagues, and lab-mates that I have had the pleasure of working with and learning from over the past five years. The opportunity to work with individuals from a variety of different backgrounds with various expertise enriched 
my experience and allowed me to learn how to work with and contribute to an exceptional team of diverse individuals. The work presented here could not have been completed without the dedicated work and contributions of my peers.

\begin{abstract}
A major challenge associated with delivery of active agents in the female reproductive tract (FRT) is the ability of agents to efficiently diffuse through the cervicovaginal mucosa
\end{abstract}


$(\mathrm{CVM})$ and reach the underlying sub-epithelial immune cell layer and vasculature. A variety of drug delivery vehicles have been employed to improve the delivery of agents across the CVM and offer the capability to increase the longevity and retention of active agents to treat infections of the female reproductive tract. Nanoparticles (NPs) have been shown to improve retention, diffusion, and cell-specific targeting via specific surface modifications, relative to other delivery platforms. In particular, polymeric NPs represent a promising option that has shown improved distribution through the CVM. This work summarizes recent experimental studies that have evaluated NP transport in the FRT, and highlights research areas that more thoroughly and efficiently inform polymeric NP design, including mathematical modeling.

The studies presented below further expand on this to investigate the application of NPs in treating cancers found within the FRT. Advanced stage cancer treatments are often invasive and painful - typically comprised of surgery, chemotherapy, and/or radiation treatment. In addition to the poor transport associated with intravaginal delivery, low transport efficiency during systemic chemotherapy may require high chemotherapeutic doses to effectively target cancerous tissue, resulting in systemic toxicity. Nanotherapeutic platforms have been proposed as an alternative to more safely and effectively deliver therapeutic agents directly to tumor sites. However, cellular internalization and tumor penetration are often diametrically opposed, with limited access to tumor regions distal from vasculature, due to irregular tissue morphologies. To address these transport challenges, NPs are often surface-modified with ligands to enhance transport and longevity after localized or systemic administration. In the work presented below, the effect of surface modification with stealth polyethylene-glycol (PEG), cell-penetrating (MPG), and CPP-stealth (MPG/PEG) poly(lactic-co-glycolic-acid) (PLGA) NP co-treatment strategies on NP distribution and chemotherapeutic efficacy, which is defined in this work as the 
ability of NPs to impart drug cytotoxicity and potency, was evaluated with the use of 3D cell culture models representing hypo-vascularized cervical cancerous tissue.

TABLE OF CONTENTS

APPROVAL PAGE .............................................................. ii

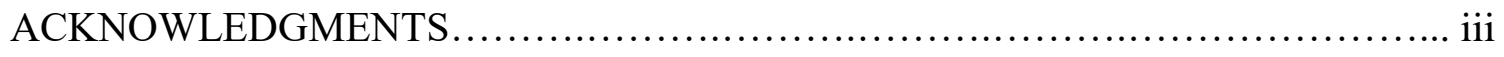

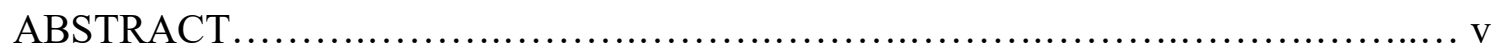

TABLE OF CONTENTS ....................................................vii

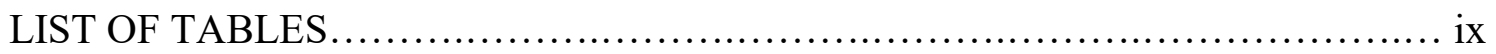

LIST OF FIGURES ......................................................... .

I. Nanoparticle-Mediated Drug Delivery to Treat Infections in the Female Reproductive Tract: Evaluation of Experimental Systems and the Potential for Mathematical Modeling. A. CURRENT CHALLENGES TO CONVENTIONAL DRUG DELIVERY ........................................................... 
B. INFORMING NANOPARTICLE DESIGN WITH EMPIRICAL

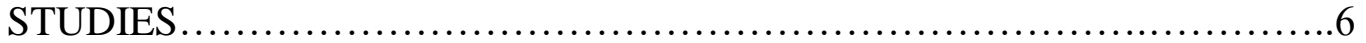

i. Introduction to factors that guide experimental studies

ii. In vitro transport studies

a. HUMAN CVM STUDIES

b. SYNTHETIC MUCUS STUDIES

c. TOWARD MORE REALISTIC IN VITRO SYSTEMS

iii. Ex vivo transport studies

iv. In vivo transport studies

a. EFFECTS OF SURFACE-MODIFICATION TYPE AND DENSITY ON IN VIVO TRANSPORT

b. EVALUATION OF TRANSPORT OF UNMODIFIED NPS

c. EFFECTS OF SURFACTANT ON IN VIVO TRANSPORT

d. EFFECTS OF MUCUS PROPERTIES AND ADMINISTRATION SOLUTION ON IN VIVO TRANSPORT

e. EFFECTS OF POLYMER TYPE ON IN VIVO TRANSPORT

f. EFFECTS OF THE REPRODUCTIVE ENVIRONMENT ON IN VIVO TRANSPORT

g. SUMMARY OF IN VIVO STUDIES

C. MATHEMATICAL MODELING OF SMALL MOLECULE DIFFUSION WITHIN THE FRT.

i. Application of mathematical modeling

ii. Modeling of drug molecule diffusion

iii. Modeling of diffusion of antibodies

iv. Definition of key parameters

v. Summary of modeling of small molecule transport through the FRT

D. UTILIZING EMPIRICAL STUDIES AND MATHEMATICAL MODELING AS COMPLEMENTARY TOOLS TO INFORM NP DESIGN ...............35

II. Enhanced uptake and transport of PLGA-modified nanoparticles in cervical cancer

A. CLINICAL SIGNIFICANCE AND BACKGROUND ..................40

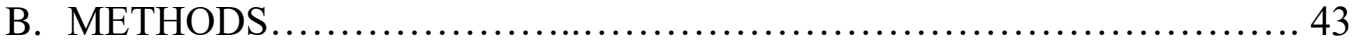

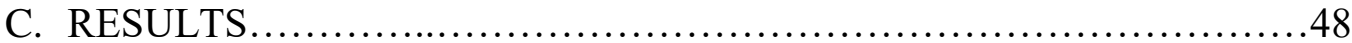

D. DISCUSSION............................................... 56

III. Distribution of PLGA-modified nanoparticles in 3D cell culture models of hypovascularized tumor tissue

A. CLINICAL SIGNIFICANCE AND BACKGROUND ....................61

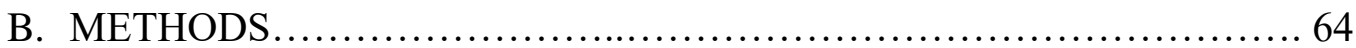

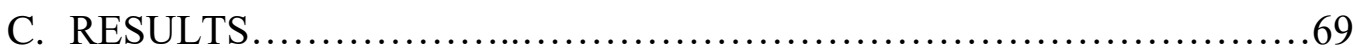

D. DISCUSSION ................................................. 80

IV. Efficacy of Surface-Modified PLGA Nanoparticles to Target Hypo-Vascularized Cervical Cancer Lesions

A. CLINICAL SIGNIFICANCE AND BACKGROUND ....................87

B. METHODS ........................................................ 92

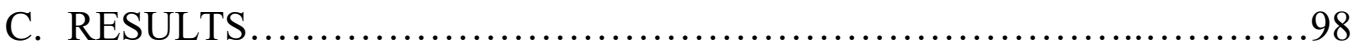

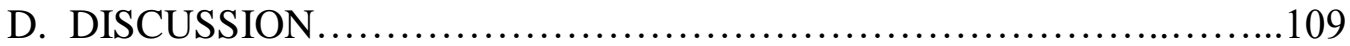




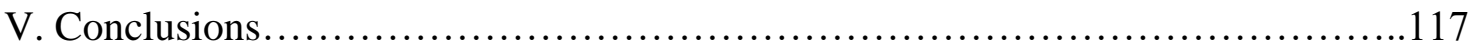

VI. References .............................................................119

\section{LIST OF TABLES}

TABLE I.1- Overview of microbicide delivery vehicles currently used or investigated for delivery to the FRT.

TABLE I.2 - Summary of mass transport equations used to model molecule diffusion in the FRT.

TABLE III.1 - Zeta potentials of unmodified, PEG-, and MPG-modified NPs. ${ }^{1}$

TABLE III.2 - Maximum cross-sectional areas of hanging drop (HD) and liquid overlay (LO) tumor spheroids as a function of cell type. ${ }^{1}$

TABLE III.3 - NP distribution represented as area-under-the-curve (AUC) (MFI- $\mu \mathrm{m})$ for each spheroid (LO or HD) and tumor cell type, relative to NP treatment group. ${ }^{1}$ 
TABLE III.4 - NP distribution in terms of maximum mean fluorescence intensity (MFI) observed at a given penetration depth $(\mu \mathrm{m})$ for each spheroid (LO or HD) and tumor cell type, relative to NP treatment group. ${ }^{1}$

TABLE IV.1 - Nanoparticle loading and encapsulation efficiency.

TABLE IV.2 - IC50 Values of Surface-Modified Dox NPs and Free Dox in Cervical Cancer Monolayers and Tumor Spheroids Expressed in Terms of Dox Concentration.

Table IV.3 - Comparison of NP efficacy (measured as IC50 in this study) to NP distribution (quantified as area-under-the-curve, AUC (MFI- $\mu \mathrm{m})$, in ${ }^{1}$ ) and maximum penetration (measured as mean fluorescence intensity (MFI) as a function of maximum penetration depth $(\mu \mathrm{m})$, in $\left.{ }^{1}\right)$ in $3 \mathrm{D}$ cell culture of various cervical cancer cell types.

\section{LIST OF FIGURES}

FIGURE I.1 - Schematic cross-section of the FRT. Mucus (top), epithelium (middle) and stromal layer containing immune cells and fibroblasts (bottom).

FIGURE I.1 - (A) Schematic of unmodified (blue only) and surface-modified mucoadhesive (yellow) and muco-inert (green) NPs. (B) Representative distribution of mucoadhesive and muco-inert NPs in the vaginal mucosa.

FIGURE II.1 - Schematic illustrating NP formulations used in this study. ${ }^{2}$

FIGURE II.2 - Cellular association and internalization of the various NP formulations are presented for HeLa (top) and VK2 (bottom) cells, shown at $1.5 \mathrm{~h}$ (left) and $24 \mathrm{~h}$ (right). Statistically similar results are shown linked with an overbar. ${ }^{2}$ 
FIGURE II.3 - Total NP association (binding and internalization) in monolayers of HeLa cells after $24 \mathrm{~h}$ incubation. Nuclei are blue (Hoechst), actin cytoskeletons are red (Texas red phalloidin), and NPs are green (Coumarin 6). ${ }^{2}$ Bar $50 \mu \mathrm{m}$

FIGURE II.4 - Total NP association (binding and internalization) in monolayers of VK2 cells after $24 \mathrm{~h}$ incubation. Nuclei are blue (Hoechst), actin cytoskeletons are red (Texas red phalloidin), and NPs are green (Coumarin 6). ${ }^{2}$ Bar $50 \mu \mathrm{m}$

FIGURE II.5 - Cellular association and internalization of the various NP formulations in HeLa cell tumor spheroids. Results are shown at (a) 1.5 and (b) $24 \mathrm{~h}$. Statistically similar results are shown linked with an overbar ${ }^{2}$

FIGURE II.6 - Comparison of NP cellular internalization between HeLa monolayer and spheroid cell cultures. ${ }^{2}$ Results are shown at (a) 1.5 and (b) $24 \mathrm{~h}$

FIGURE II.7 - NP penetration into 3D cell culture (HeLa spheroids) after $1.5 \mathrm{hr}$ incubation. ${ }^{2}$

FIGURE III.1 - Schematic of spheroid formation techniques for a liquid overlay (LO) and b hanging drop (HD) spheroids, respectively representing larger, more irregularly-shaped and smaller, regularly-shaped avascular tissue. ${ }^{1}$

FIGURE III.2 - Typical morphologies of liquid overlay (LO) and hanging drop (HD) tumor spheroids, evaluated via bright feld microscopy. ${ }^{1}$ Scale bar: $200 \mu \mathrm{m}$

FIGURE III.3 - NP distribution through liquid overlay (LO) spheroids in mid-plane crosssections (top three rows) and 3D composite (bottom three rows) confocal images. Nuclei are blue (Hoechst) and NPs are green (Coumarin 6). ${ }^{1}$ Scale bar: $50 \mu \mathrm{m}$

FIGURE III.4 - NP distribution through hanging drop (HD) spheroids in mid-plane crosssections (top three rows) and 3D composite (bottom three rows) confocal images. Nuclei are blue (Hoechst) and NPs are green (Coumarin 6). ${ }^{1}$ Scale bar: $50 \mu \mathrm{m}$

FIGURE III.5 - NP distribution profles quantifying the mean fuorescence intensity (MFI) vs. penetration distance through liquid overlay (LO) spheroids. Distribution profles are shown as a function of NP treatment and tumor cell type. Average of the values along distance is denoted by the dark lines. ${ }^{1}$

FIGURE III.6 - NP distribution profles quantifying the mean fuorescence intensity (MFI) vs. penetration distance through hanging drop (HD) spheroids. Distribution profles are shown as a function of NP treatment and tumor cell type. Average of the values along distance is denoted by the dark lines. ${ }^{1}$

FIGURE III.7 - NP distribution represented as AUC for each tumor cell type (HeLa, SiHa, or CaSki) as a function of NP treatment group, relative to spheroid type (LO, black and HD, gray). Values of all signifcant correlations, including each treatment group relative to unmodi- fed NPs, relative to other treatment groups, or relative to the same treatment group in a diferent spheroid type are given with degree of signifcance indicated $(* \mathrm{p}<0.01, * * \mathrm{p}<0.001, * * * \mathrm{p}<0.0001$, $* * * * \mathrm{p}<0.00001)$. Error bars: average \pm standard deviation $(\mathrm{n}=3)^{1}$ 
FIGURE IV.1 - The cumulative release of Dox from unmodified (triangle), MPG (circle), and PEG (square) modified NPs, as a function of (A) NP mass and (B) percent total loading.

FIGURE IV.2 - Fluorescent cross-sectional images of cervical tumor spheroids comprised of (A) HeLa, (B) SiHa, and (C) CaSki cells stained with Hoechst dye.

FIGURE IV.3 - NP association (binding plus internalization, black) and internalization (grey) in 3D tumor spheroids after $1.5 \mathrm{hr}$ (left) and $24 \mathrm{hr}$ (right) administration.

FIGURE IV.4 - The cytotoxicity of surface-modified Dox NPs and free Dox in HeLa, SiHa, and CaSki cervical carcinoma monolayers after $24 \mathrm{hr}$ administration, as a function of Dox dose.

FIGURE IV.5 - The cytotoxicity of surface-modified Dox NPs and free Dox in HeLa, SiHa, and CaSki cervical carcinoma 3D tumor spheroids after $24 \mathrm{hr}$ administration, as a function of Dox dose.

FIGURE IV.6 - The IC50s of surface-modified NPs after $24 \mathrm{hr}$ administration in cervical carcinoma monolayers (top) and 3D tumor spheroids (bottom) as a function of Dox (A and C, $\mu \mathrm{M}$ ) and NP (B and $\mathrm{D}, \mathrm{mg} / \mathrm{mL}$ ) concentrations. 
I. Nanoparticle-Mediated Drug Delivery to Treat Infections in the Female Reproductive Tract: Evaluation of Experimental Systems and the Potential for Mathematical Modeling

\section{Adapted From:}

Sims LB, Frieboes H, Steinbach-Rankins JM; Nanoparticle-Mediated Drug Delivery to Treat Infections in the Female Reproductive Tract: Evaluation of Experimental Systems and the

Potential for Mathematical Modeling; 2018; Accepted

\section{A. CURRENT CHALLENGES TO CONVENTIONAL DRUG DELIVERY}

Mucosal tissue is a critical barrier that defends the body from pathogen infection, typically lining the surface of internal organs and body cavities. Within the female reproductive tract (FRT), the vaginal mucosa is comprised of three layers that possess unique characteristics relevant to their protective functions (Figure 1). ${ }^{3,4}$ The secreted mucus layer, located on the apical side of the mucosa, is comprised of polymerized mucin fibers and globular secretions. Cervical mucus is produced by the ectocervix and forms cervicovaginal mucus (CVM) that locally lines the cervix, as well as the vaginal compartment. Beneath the mucus, within the vaginal compartment, lies the stratified epithelium, which is

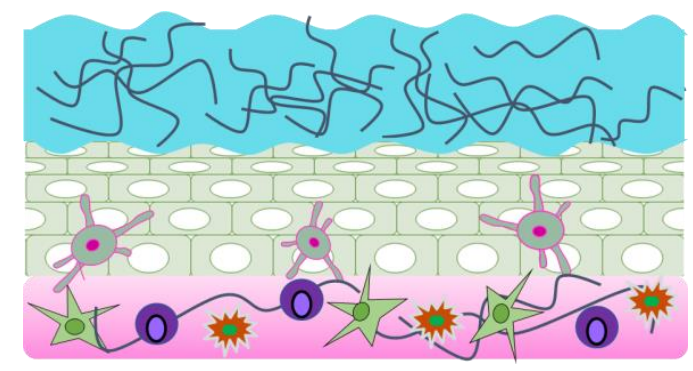

Figure I. Schematic cross-section of the FRT. Mucus (top), epithelium (middle) and stromal layer containing immune cells and fibroblasts (bottom). approximately $200-400 \mu \mathrm{m}$ thick. The lamina propria (or stroma) lies at the basal portion of the mucosa and is the thickest $(2.5-3 \mathrm{~mm})$ and most complex of the three layers. Similar to the apical mucus, an array of mucin fibers can also be found within the stroma. ${ }^{5}$ The arrangement of these mucin fibers, in parallel fiber networks or more randomized patterns, is influenced by menstrual and ovulation cycles. In addition, the stroma contains immune (e.g., CD3+ and CD4+ T lymphocytes) and host (e.g., macrophage, fibroblast) cells that are recruited to mediate the host-antimicrobial response.

The physiological and anatomical complexity of the FRT maintains the health of the microbiota and host cells by acting as a first line of defense against incoming pathogens. Yet the 
effectiveness of the CVM as a barrier to infection can present significant challenges to the localized administration of microbicidal, antiviral, and antibiotic agents. Traditional dosage forms and delivery vehicles that deliver prophylactic and therapeutic active agents to the FRT, such as intravaginal rings, gels, and films face numerous obstacles including: rapid clearance and dilution of topically applied active agents due to vaginal fluids, minimal diffusion through the CVM, poor transport to underlying tissue, and inadequate, heterogeneous agent distribution. To address these challenges, a variety of platforms have been developed to deliver therapeutic agents across the CVM, while also increasing the longevity and retention of active agents in the FRT. These platforms offer numerous advantages over traditional dosage forms by protecting active agents from degradation in the harsh acidic vaginal microenvironment, providing sustained-release to improve the longevity and efficacy of the therapeutic payload, as well as enabling the high encapsulation and localized delivery of hydrophobic and hydrophilic agents (Table 1). For a more thorough review of microbicide delivery platforms please refer to ${ }^{6-11}$. 
Table 1. Overview of microbicide delivery vehicles currently used or investigated for delivery to the FRT.

\begin{tabular}{|c|c|c|c|}
\hline $\begin{array}{l}\text { Microbicide Delivery } \\
\text { Platform }\end{array}$ & Attributes & Challenges & Schematic Representation \\
\hline Intravaginal Rings & Lead in sustained-delivery, avoid leakage, coitus-independent & $\begin{array}{l}\text { Incorporation of biologics, } \\
\text { alternative delivery vehicle }\end{array}$ & \\
\hline Films & Convenient, portable, discreet, no leakage, low cost & Often transient release & \\
\hline Gels & $\begin{array}{l}\text { Convenient, easy to manufacture, mucosal adhesion and } \\
\text { spreading, comfort attributed to water content }\end{array}$ & $\begin{array}{l}\text { Often transient activity, } \\
\text { messiness due to leakage }\end{array}$ & \\
\hline Nanoparticles & $\begin{array}{l}\text { Sustained-delivery, cell-specific targeting, incorporate } \\
\text { biologics, mobile platform for cell/tissue penetration and } \\
\text { uptake, potential as a coitus-independent platform }\end{array}$ & $\begin{array}{l}\text { Messiness and NP loss due to } \\
\text { leakage }\end{array}$ & \\
\hline Electrospun Fibers & $\begin{array}{l}\text { Potential for sustained-delivery, alternative delivery platform, } \\
\text { potential as coitus-independent platform }\end{array}$ & $\begin{array}{l}\text { User preference and comfort } \\
\text { unknown }\end{array}$ & \\
\hline
\end{tabular}

A promising platform that has been utilized to improve distribution through the CVM is based on polymeric nanoparticles (NPs). ${ }^{1,2,7-9,11-37}$ Due to small sizes, often ranging from 70 to $300 \mathrm{~nm}$,

NPs exhibit unique physical properties that make them advantageous for delivery to the FRT, often providing desirable diffusion and transport kinetics through the CVM. By incorporating different surface modifications, NPs can improve retention, diffusion, and cellspecific targeting, relative to other delivery platforms. Additionally, polymeric NPs are typically made from non-toxic, noninflammatory, FDA-approved polymers, which
A.

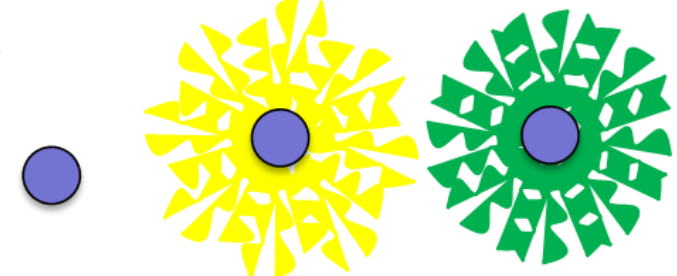

Unmodified Mucoadhesive Muco-Inert

B.

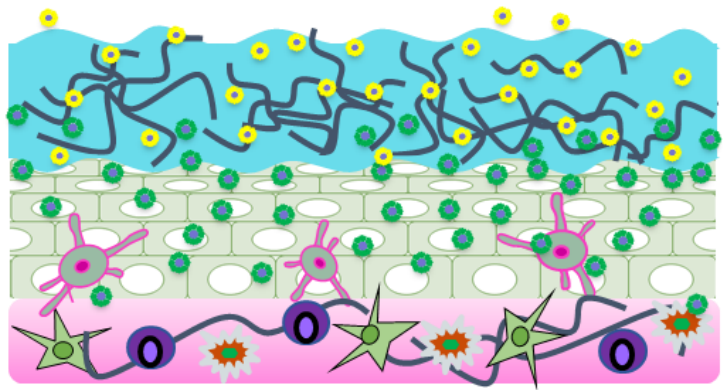

Figure 2. (A) Schematic of unmodified (blue only) and surface-modified mucoadhesive (yellow) and muco-inert (green) NPs. (B) Representative distribution of mucoadhesive and muco-inert NPs in the vaginal mucosa. 
improve biocompatibility - a factor of significant importance due to the propensity for vaginal infections. The nanoscale properties, including high surface area to volume ratio, can impart favorable tunability in transport properties. This is achieved through NP surface modifications, by utilizing different surface chemistries to conjugate or adsorb ligands to the NP surface. These modifications can either enhance NP diffusion through the CVM via muco-inert properties, or can impart mucoadhesive characteristics for greater longevity in the basal mucus layer (Figure 2)..$^{7,20,21,25,31,32,36,38-40}$ Hence, NPs can directly carry therapeutic payloads and navigate through the porous CVM, increasing agent penetration and transport. Moreover, alterations in physicochemical properties can be adapted to attain desired pharmacokinetic (PK) and pharmacodynamic (PD) properties. ${ }^{1,2,8,9,12,16,17,21,25,36,39,41}$ Thus, polymeric NPs can be designed to overcome transport barriers and to deliver effective doses to target sites, while maintaining agent activity and minimizing adverse effects on surrounding tissue.

To more effectively design delivery vehicles for application to the FRT, an in-depth understanding is needed of the anatomical and physiological structure of the vaginal microenvironment and how these structural characteristics may directly or indirectly affect NP diffusion and transport. A variety of studies have examined the impact of mucin fibers, found in the apical and basal layers of the mucosa, on NP distribution and transport. ${ }^{14,42}$ Mucin, a major structural component of mucus, is a high molecular weight, anionic molecule that non-covalently interacts to create a mesh-like framework. Depending on the arrangement, size, and porosity of these mucin fibers, active agent and delivery vehicle penetration are impeded, similar to virus penetration. ${ }^{43-45}$ Additionally, the affinity for NPs to reversibly bind to mucin fibers has been studied and shown to impact NP diffusion. These studies, discussed in more detail below, provide insight into the dependence of diffusion through the mucosa on ligand choice, surface 
modification density, and ligand molecular weight. Although NP diffusion and transport, as a function of these alterations, have been experimentally studied in vitro and in vivo, limitless alterations exist, making it difficult to iteratively test all of the parameters that can impact binding, diffusion, and internalization.

In parallel with experimental design, mathematical modeling has been used to validate and predict factors that play pivotal roles in drug and NP distribution, particularly in the field of cancer. Mathematical models have been used to obtain more detailed insights into how specific parameters including size, shape, surface modifications, and drug loading affect NP diffusion, without having to perform exhaustive experiments to inform design. ${ }^{46-68}$

The goal of this chapter is to highlight recent experimental studies that have evaluated NP transport in the FRT. As there have been to our knowledge no mathematical studies evaluating NP transport in the FRT, we focus on modeling studies that have evaluated small molecule transport, and describe research parameters and regimens that may be expanded upon to more thoroughly and efficiently inform potential NP design. We first present an overview of the in vitro, ex vivo, and in vivo polymeric NP studies conducted to date, by which transport parameters are determined, extrapolated, and validated. Secondly, we summarize how different NP design features have impacted FRT transport, and identify gaps that exist due to the limitations of iterative experimentation alone. We then discuss the potential of mathematical modeling as a complementary means to characterize and evaluate diffusion and transport of delivery vehicles and active agents through the CVM. Lastly, we suggest potential advancements combining 
mathematical and experimental knowledge to inform next-generation designs, such that optimal NP binding, diffusion, and internalization in the FRT may be achieved.

\section{B. INFORMING NANOPARTICLE DESIGN WITH EMPIRICAL STUDIES}

i. Introduction to factors that guide experimental studies

As the field of intravaginal drug delivery has evolved, a variety of experimental methods have been established to study molecular and NP transport in vaginal mucosa models. In vitro, ex vivo, and in vivo methods have provided insight into the pharmacologic behaviors of molecules and delivery vehicles, particularly their movement through the CVM. Each type of experimental model provides information regarding transport and the interactions that modulate this transport, with varying degrees of physiological complexity and accuracy.

The most controlled methods to study molecular diffusion and transport are provided by in vitro experimental models, in which individual cell uptake and effect can be evaluated. However, monolayer cell culture often fails to adequately represent a system in which NPs undergo diffusive flux as they would in vivo, due to the inability of these systems to accurately represent 3D physiologic architecture and microenvironmental conditions. To more faithfully represent the mucosal environment, more advanced in vitro systems such as synthetic mucus, ${ }^{69}$ undiluted CVM collected from human patients, ${ }^{13,22,30,31,35,70-72}$ and 3D cell culture techniques have been developed. . $28,52,55,73-77$

In addition to utilizing a relevant model to study and obtain transport information, essential physical and chemical properties must be considered to effectively design NPs to navigate the CVM. Depending on whether mucoadhesive or muco-inert properties are preferred, these 
properties may be tailored to enhance distribution through mucus and to the underlying epithelial and stromal cell layers. A particular physical parameter, NP size, has been found to impact NP diffusion and transport kinetics, due to the pore size of the surrounding CVM. ${ }^{3,22,23,42}$ If NPs are larger than the average CVM pore diameter, effective transport may be hindered by physical and chemical interactions with mucin fibers. Contributing to these interactions, NP surface chemistry also impacts NP transport: surface charge, colloidal stability, surface-modifying agent of choice, molecular weight, and ligand surface density all affect NP interactions with the mucosa. ${ }^{13,20-}$ 22,26,27,30,31,34,35,37,40,43,72,78 These factors can influence how NPs, in a concentration-dependent manner, interact with one another.

Together, these factors contribute to the complexities associated with first understanding NP diffusion within the CVM, and subsequently designing optimal NP delivery vehicles to meet the needs of a given application or physiological environment. Previous literature reviews ${ }^{7-}$ 9,11,17,19,27,32,38 have focused on the therapeutic efficacy of NP delivery systems and their potential applications in the FRT environment. Here, we highlight different in vitro, ex vivo, and in vivo techniques that have been implemented to better understand how the relevant parameters influence NP diffusion and transport kinetics in the CVM. In this section we present these techniques and summarize how they have been utilized to gain deeper insights into the complexities of NP delivery within the FRT.

ii. $\quad$ In vitro transport studies

a. HUMAN CVM STUDIES

Transport dependence on NP size and surface modifications 
Within the field of intravaginal drug delivery, CVM samples are often collected from patients and serve as important specimens to study NP transport and diffusion. This methodology provides a near perfect physiological mucus model in which NP transport may be studied..$^{4,14,20,22,23,30,31,35,70,71}$ In a seminal study by Saltzman and group, fresh, mid-cycle cervical mucus was obtained from healthy human patients and used to investigate how mucus properties change based on interactions with synthetic polymers. ${ }^{72}$ In the study, the addition of nonionic or cationic polymers such as polyethylene glycol (PEG) or polyvinylpyrrolidone (PVP) altered the gel-like structure of mucus. It was discovered that the addition of PEG (3350 Da) affected the structural properties of mucus by inducing hydrogen bond formation, leading to mucin fiber coalescence in regions that were in contact with the polymer, thereby creating larger pore sizes. In contrast, the addition of anionic polymers like poly(lactic-co-glycolic) acid (PLGA), had no effect on the mucus properties. ${ }^{72}$ The inability of PLGA to induce structural changes within CVM may correlate with the observation that PLGA NPs tend to immobilize within the CVM, while, conversely, the ability of PEG to alter mucin fiber arrangement may contribute to the enhanced diffusivity of PEG-modified NPs, as discussed in greater detail below. The results from this pioneering study highlight the impact of polymer selection on NP-CVM interactions. ${ }^{72}$

In later work, human CVM was used in vitro to investigate the diffusion kinetics of different polymeric NP formulations, including polystyrene (PS) and PLGA NPs..$^{22,23,29-31,34,35,37,79}$ In these studies, undiluted CVM secretions were collected from women who displayed a healthy vaginal flora,. Utilizing these specimens, Hanes and group investigated the relative diffusivities of different NP formulations. ${ }^{22,23,29-31,34,35,37,79}$ Parameters that were of particular interest were NP size and the molecular weight and surface density of NP modifications, such as PEG. In one such 
study investigating the physical properties of NP size and surface charge, it was found that PS NPs, with diameters spanning 200-500 nm, exhibited more rapid diffusion independent of surface chemistry and charge, relative to smaller, $100 \mathrm{~nm} \mathrm{NPs.}{ }^{22}$ This was contrary to the hypothesis that NPs with a diameter larger than the CVM pore size would have lower diffusion coefficients, relative to NPs with diameters smaller than the average pore size. Polystyrene NPs modified with PEG (PEGylated, $2 \mathrm{kDa}$ ) ranging in size from 100, 200, and $500 \mathrm{~nm}$ exhibited 20, 400-, and 1,100-fold higher average mean squared displacements (MSDs) relative to unmodified carboxylated (PS-COOH) NPs of the same size. Furthermore, NPs with larger diameters and dense PEG coatings diffused through the CVM more rapidly, with diffusion coefficients reduced by 2,000-, 6-, and 4-fold for 100, 200, and $500 \mathrm{~nm}$ PEGylated NPs, relative to diffusion in water. In contrast, it was observed that PS-COOH NPs (without modification) were unable to diffuse through the CVM and were strongly mucoadhesive. ${ }^{22}$ This phenomenon was attributed to the addition of PEG, preventing polyvalent bond formation between PS NPs and hydrophobic mucin fiber domains. From this study it was found that NP surface chemistry plays a vital role in diffusion through CVM.

To further explore the impact of NP size on CVM diffusion, a separate study investigated how the nanoscale mucus rheology changes upon exposure to differently sized non-mucoadhesive beads. ${ }^{70}$ In fresh, undiluted human CVM, the mucosa acted as an impermeable elastic barrier to non-mucoadhesive beads with diameters equal to or greater than $1 \mu \mathrm{m}$, while the CVM resembled a highly permeable viscoelastic liquid for beads with a diameter less than or equal to $500 \mathrm{~nm}$. However, after addition of a nonionic detergent, the CVM transitioned to an impermeable elastic barrier, even for the small beads. ${ }^{70}$ This change in mucus viscoelasticity is in 
accordance with the results from ${ }^{22}$, suggesting that NPs with diameters spanning 200 to $500 \mathrm{~nm}$, achieve maximum diffusion.

To gain further insight regarding the role of mucin fiber pore size in NP diffusion and to expand on the study by ${ }^{22}$, experiments were conducted to investigate the effect of size on NP penetration through the CVM. ${ }^{23}$ Polystyrene NPs were modified with a high density, low molecular weight, amine-modified PEG (2.0-3.4 kDa) to deter mucus adhesion. When observed in undiluted human CVM, PEG-modified PS NPs with sizes 100, 200, and $500 \mathrm{~nm}$ effectively penetrated mucus and exhibited diffusivities 6.5-, 3.5-, and 12-fold lower than that of NPs in water, respectively. However, PEG-modified PS NPs with a diameter of $1 \mu \mathrm{m}$ diffused 200-fold more slowly in mucus than in water. Using this information, the diffusion rates were fit to a previously derived obstruction-scaling model for cross-linked gels to determine the pore size of CVM. $^{23,80}$ While the pore size of undiluted human CVM was found to be $340 \pm 70 \mathrm{~nm}$, this wide distribution highlights the additional complexities of transport in the CVM. Additionally, the large difference in diffusion coefficients between the 0.5 and $1 \mu \mathrm{m}$ PS NPs emphasizes the importance of NP diameter in NP design.

In addition to NP size, ligand density and surface modification type play critical roles in the transport of NPs in human CVM. The effect of PEG as a surface-modification for PLGA NPs and its impact on CVM binding and diffusion were investigated as a function of PEG molecular weight $(2,5$, and $10 \mathrm{kDa})$ and modification density (5-100\%). ${ }^{14}$ As predicted by the investigators, the addition of PEG to the NP surface impacted the physical properties of the NPs by improving colloidal stability and neutralizing the NP surface charge. PEGylated NPs were 
also observed to have higher diffusion coefficients in human cervical mucus. Additionally, NPs partially-modified with PEG (10\%) had a higher dependency on PEG molecular weight, whereas NPs partially-modified (10\%) with $10 \mathrm{kDa}$ PEG exhibited no difference relative to fullymodified PEGylated NPs using 2 and 5 kDa PEG. ${ }^{14}$ Based on PEG molecular weight, the partially-modified PEGylated NP formulations (10\%) with 5 or $10 \mathrm{kDa}$ PEG demonstrated statistically improved CVM diffusion relative to unmodified and $2 \mathrm{kDa}$ partially-modified PLGA NPs. These results highlight the interdependency of PEG molecular weight and modification density on NP diffusion through CVM.

\section{Transport dependence on NP formulation chemistry and synthesis}

As high PEG-modification density was observed to promote mucus penetration and decrease NP immobilization, similar studies sought to identify ways to maximize ligand conjugation to the NP surface. $^{30,37}$ One of the challenges in obtaining high degrees of post-synthesis surface modification is that with conventional polymers, such as PS and PLGA, there are a limited number of surface groups available for conjugation, thereby limiting the density at which PEG (and other ligands) can be conjugated to the NP surface. To overcome this obstacle, PEG was covalently conjugated to the backbone of poly(sebacic acid) (PSA) to create a diblock copolymer that resulted in muco-inert NPs. ${ }^{30}$ The trajectories and diffusion coefficients of PSA-PEG, relative to unmodified PSA and PLGA NPs were observed in undiluted human CVM. PSA-PEG NPs were significantly more mobile in CVM, having a MSD 400- and 230-fold higher than PSA and PLGA NPs, respectively, confirming the correlation between high PEG density and rapid NP diffusion and transport through the CVM. 
Another study investigated the use of biodegradable polymers to formulate diblock copolymers of PLGA (18 kDa; L/G=50:50) and PEG (2 kDa) to promote mucus-penetrating NP properties. ${ }^{37}$ The chemical structure of the diblock polymer, confirmed via H-NMR and NP characterization with dynamic light scattering, revealed that PLGA and PLGA-PEG NPs possessed average hydrodynamic diameters of 130 and $90 \mathrm{~nm}$, respectively. To assess NP diffusion, fresh undiluted CVM was collected from human patients and in this system, PLGA-PEG NPs were observed to diffuse much faster than PLGA NPs alone, with diffusion coefficients 8,000 and 12,000-fold lower than in water, respectively. Based on these results, it was suggested that $\sim 75 \%$ of PLGAPEG NPs would penetrate a $10 \mu \mathrm{m}$ thick mucus layer in $30 \mathrm{~min}$, whereas PLGA NPs would exhibit negligible penetration within this duration. ${ }^{37}$ Due to the rapid clearance of the CVM - on the order of minutes to hours - it is highly desirable that topically administered NPs penetrate and diffuse into tissue prior to mucosal clearance. Furthermore, differences in the thickness of mucosal linings arising from different physiologic factors such as age and stage of the menstrual cycle, vary from patient to patient. Therefore, these findings suggest that PEGylated NPs, created by pre-synthesis copolymerization, may avoid mucosal clearance while still providing adequate diffusion and penetration within the vaginal tissue. Both of these studies reveal the impact that two different copolymer blends can have on formulating mucus-penetrating NPs while eliminating the need of post-synthesis surface modification. ${ }^{30,37}$

\section{Transport dependence on NP formulation reactants}

The impact of residual reactants from NP synthesis was explored in relation to NP transport through the CVM. ${ }^{37,40,79}$ Surfactants that are typically used for NP synthesis, such as polyvinyl alcohol (PVA), are intrinsically mucoadhesive, and therefore post-synthesis residue may impede 
NP transport through mucus. Given this, the effect of surfactants on CVM distribution was assessed by analyzing NP diffusion in human CVM specimens. PLGA NPs were synthesized with novel vitamin E surfactants that had been previously conjugated to a 1 or $5 \mathrm{kDa}$ methoxyPEG-OH (PLGA/VP1K and VP5K, respectively), PVA surfactant alone, and COOH-only modified (PS-COOH) or PEG-modified (2 kDa) PS NPs as controls. ${ }^{40}$ PLGA NPs synthesized using the VP1K surfactant exhibited no marked increase in CVM transport relative to unmodified COOH-only PS NPs. ${ }^{40}$ However, increasing the PEG molecular weight to $5 \mathrm{kDa}$ significantly impacted diffusion. The MSD of PLGA/VP5K NPs in CVM was 210- and 33-fold higher than that of PS-COOH and PLGA/VP1K NPs, respectively. Moreover, VP5K PLGA NPs were found to be the most resistant to mucoadhesion relative to VP1K and traditional PLGA NPs, confirming the importance of and sensitivity to surfactant and PEG molecular weight on CVM diffusion. ${ }^{40}$

Given the correlation between neutral surface modification and enhanced mucus penetration, another study sought to investigate the effect of PVA alone on CVM transport. Due to the hydrophilic and neutral nature of PVA, it was hypothesized that coating PLGA and PS NPs with PVA may enhance NP diffusion. ${ }^{79}$ Interestingly, PS NPs coated with PVA, regardless of concentration $(0.01$ or $1 \% \mathrm{w} / \mathrm{v})$ or molecular weight $(2,6$, or $25 \mathrm{kDa})$ remained immobilized in the CVM. When tested in fresh, undiluted human mucus samples, PS NPs that were synthesized with 2, 6, and $25 \mathrm{kDa}$ PVA (1\% w/v incubation concentration) had diffusion coefficients 23,000, 14,000-, and 10,000-fold lower than their theoretical values in water, respectively. ${ }^{79}$ The effect of increasing PVA molecular weight decreased mucoadhesion and therefore increased diffusion. While PLGA NPs formulated with PVA surfactants were immobilized in the CVM relative to the 
surfactant-free NPs and PEG-PLGA NPs, these results suggest that the inclusion of PVA as a surfactant or surface modification may promote mucoadhesivity that can be tailored with changes in molecular weight or concentration.

\section{Summary of human CVM studies}

The use of human CVM affords the possibility to emulate a physiologically relevant mucosal environment and to use this environment to gain an understanding of how physicochemical parameters impact NP diffusion in the CVM. While in vitro studies that utilize human CVM studies provide an informative system, there are drawbacks to this methodology. Sources of human CVM are not always readily available, and adequate amounts of CVM required to study multiple diffusion parameters can be challenging to obtain. Furthermore, patient samples are often inherently different from one another. In spite of these drawbacks, utilizing human CVM is a relatively straightforward technique that allows for rapid data interpretation, which can be valuable to the design of NP formulations. With this technique, NP delivery vehicles can be precisely designed to obtain mucoadhesion (for retention) or mucus penetration (for distribution). By using human CVM, the reviewed studies were able to explore how NP size, formulation chemistry, formulation reactants, surface charge, surface-ligand density, ligand molecular weight, and surfactant may significantly impact NP diffusion in an environment closely corresponding to in vivo CVM conditions.

\section{b. SYNTHETIC MUCUS STUDIES}

Synthetic mucus formulations have been developed to study mucus rheology and the diffusion of small molecules and some delivery vehicles in vitro as a means to avoid the shortcomings of 
human CVM. ${ }^{17,18,70}$ Although these mucus formulations are not obtained from humans, they are able to recapitulate critical mucus properties such as microstructure and viscoelasticity. Synthetic mucus has the ability to adequately simulate bulk mucus properties, even though it lacks critical components of a biologic system, such as innate physiological interactions and cellularmolecular components. While the majority of studies have focused on the analysis of mucus microstructure, ${ }^{4,27,30,42,72}$ viral transport, ${ }^{11,24,43-45,71,81}$ or small molecule diffusion, ${ }^{71,81-83}$ limited studies have used synthetic mucus to assess delivery vehicle transport.

\section{Transport dependence on NP formulation chemistry}

Most recently, the interactions of dapivirine-loaded polycaprolactone (PCL) NPs were studied in a synthetic mucus environment. ${ }^{16,18}$ Polycaprolactone NPs encapsulating dapivirine were evaluated due to their potential application as microbicides, and their demonstrated mucoadhesivity, sustained-release, and retention within the CVM. To create synthetic mucus, mucin fibers were added to SVF $(1.5 \% \mathrm{w} / \mathrm{v})$ followed by bulk rheological analysis to confirm that the viscoelastic properties were comparable to human CVM. ${ }^{18}$ Using this in vitro model, it was observed that varying NP surface charge significantly contributes to altering the diffusion time across a $200 \mu \mathrm{L}$ layer of SVF in an 8-well imaging chamber. In this system, negativelycharged NPs exhibited the most rapid diffusion. ${ }^{18}$

To expand on this work, the effect of NPs modified with cetyltrimethylammonium bromide (CTAB), poly(ethylene oxide) (PEO), and sodium lauryl sulfate (SLS) was assessed both in in vitro cell monolayers and ex vivo porcine vaginal mucosa, to increase dapivirine retention in the vaginal CVM. ${ }^{10}$ In cell monolayers comprised of CaSki vaginal epithelial cells, PEO-PCL NPs 
exhibited the lowest apparent permeability coefficient of dapivirine $\left(2.2 \pm 0.2 \mathrm{~cm} \cdot \mathrm{s}^{-1} \times 10^{-6}\right)$ relative to free dapivirine $\left(3.0 \pm 0.3 \mathrm{~cm} \cdot \mathrm{s}^{-1} \times 10^{-6}\right)$, while CTAB NPs exhibited the highest dapivirine permeability coefficient $\left(4.6 \pm 0.5 \mathrm{~cm} \cdot \mathrm{s}^{-1} \times 10^{-6}\right)$. In subsequent studies, ex vivo vaginal porcine tissue was utilized to further explore how these surface modifications impacted dapivirine retention and NP diffusion. ${ }^{16}$ Permeability coefficients obtained ex vivo followed similar trends, with PEO-PCL NPs exhibiting a very low dapivirine permeability coefficient $(0.6$ $\pm 0.2 \mathrm{~cm} \cdot \mathrm{s}^{-1} \times 10^{-6}$ ) and CTAB-PCL NPs diffusing more rapidly, with a dapivirine permeability coefficient of $3.0 \pm 0.3 \mathrm{~cm} \cdot \mathrm{s}^{-1} \times 10^{-6}$, relative to PEO-PCL NPs and free dapivirine. These results suggest that PCL NPs modified with PEO have the strongest mucoadhesion, whereas CTABPCL NPs diffuse more rapidly through the vaginal epithelium. ${ }^{16}$

\section{Transport dependence on NP modifying agent and surface charge}

Similar to the results obtained with human CVM, it was observed that NP modifying agent and surface charge significantly contribute to NP affinity for mucus. ${ }^{16}$ Interestingly, PEO-modified NPs exhibited enhanced mucoadhesion properties in this synthetic CVM system, which is in contrast to the aforementioned studies in which high molecular weight PEG (10-40 kDa) was utilized to decrease mucoadhesion. ${ }^{14,29,30}$ This discrepancy may be due to the difference between human and synthetic mucus, and highlights the complexity of NP-CVM interactions.

\section{c. TOWARD MORE REALISTIC IN VITRO SYSTEMS}

One of the main drawbacks of in vitro systems utilizing human or synthetic CVM is the challenge of accurately representing the in vivo environment, including the complex anatomical structure of the mucus and the underlying tissue that comprises the FRT. When delivered 
topically, it is often desirable for NPs to traverse mucus, withstand mucus shedding, and penetrate to the underlying epithelial and stromal tissue, depending on the intended cell or pathogen target. To complement analyses provided by human or synthetic CVM models, 3D models that include polymer matrices and multi-cellular layers, representative of epithelial and stromal layers, have been created to provide a noninvasive and physiologically relevant environment in which to study transport parameters. While a comprehensive review of available cell culture models may be found in ${ }^{74}$, hallmark studies relevant to FRT delivery include $44,69,73,74,76,77,84,85$.

\section{iii. Ex vivo transport studies}

In addition to human and synthetic mucus in vitro systems that have been employed to study NP transport, ex vivo models have been developed to gain insight into NP transport in the intravaginal environment. By integrating some of the attributes of in vitro and in vivo systems, ex vivo models can provide physiologically relevant tissue architectures, representative of the FRT microenvironment. In particular, porcine vaginal tissue has been used to investigate the mucoadhesive properties of NPs and other colloidal systems, as described in ${ }^{41,86}$.

Similar methodologies have been used to investigate the efficacy of tenofovir-encapsulated chitosan NPs as a potential microbicide. ${ }^{87}$ Chitosan NPs were synthesized with varying diameters, with the smallest and largest NPs ranging from 188 to $900 \mathrm{~nm}$. Due to the inherent bioadhesive properties of chitosan, it was hypothesized that NP mucoadhesion would be sizedependent. It was found that NPs with smaller diameters were twice as mucoadhesive relative to 
the larger NPs. ${ }^{87}$ These results are in agreement with previous work that found that PS NPs ranging from $200-500 \mathrm{~nm}$ exhibited more rapid diffusion relative to $100 \mathrm{~nm} \mathrm{NPs},{ }^{22}$ suggesting that physical properties such as size and NP material (here PS or chitosan) can be used to tailor NP mucoadhesivity.

While ex vivo systems provide a physiologically representative environment in which to study NP diffusion - having defined mucosal and underlying vaginal tissue layers - they still suffer from limitations, such as the inherent differences between animal and human tissue. Additionally, when studying diffusion in ex vivo models, there is an inability to temporally evaluate NP transport and clearance from the CVM, relative to vaginal fluid and tissue flux, since continual fluid exchange does not occur in these models.

\section{iv. In vivo transport studies}

Although in vitro systems provide a highly controlled environment, spatiotemporal knowledge is seldom derived from in vitro cell monolayer studies due to the two-dimensional limitations and inherent differences of in vitro systems relative to in vivo tissue. Additionally, an in vitro system is typically limited to one or two cells types, or is focused on mucus alone, providing a comparatively isolated and compartmentalized method to investigate NP diffusion and transport. As such, in vivo systems can address the limitations of in vitro as well as ex vivo models, and enable the comprehensive assessment of how alterations in NP formulations impact transport in the CVM. A more comprehensive evaluation is possible due to the ability of in vivo models to provide a realistic environment in which to study NP diffusion and transport, and to evaluate the 
associated therapeutic efficacy within the FRT. In particular, tissue histology from in vivo studies provides knowledge of how drug delivery vehicles diffuse and distribute throughout vaginal tissue. Such in vivo studies enable investigators to study the diffusion kinetics within the FRT as an integrated system, which provides a more thorough evaluation of how NP delivery systems interact with the CVM and vaginal tissue. With this in mind, the complexity of the in vivo environment requires the consideration of more parameters to rationally design NP delivery vehicles.

\section{a. EFFECTS OF SURFACE-MODIFICATION TYPE AND DENSITY ON IN VIVO}

\section{TRANSPORT}

One way to improve intravaginal penetration and retention of polymeric NPs in vivo has been to conjugate NPs with ligands to modulate physicochemical properties, such as surface charge and hydrophobicity. To explore the effect of conjugating biotinylated PEG (2 kDa) to avidinmodified PLGA NPs, a murine model was used to measure the vaginal retention of surfacemodified NPs. ${ }^{12}$ Nanoparticle groups including avidin-only and avidin-PEG NPs exhibited up to 5-times increased vaginal retention, relative to unmodified NPs. Furthermore, PEG NPs were found within the submucosal stromal cells and epithelium, suggesting that these NPs efficaciously penetrate the CVM to the underlying basal immune cells. Relative to avidin-only and unmodified NPs, PEG NPs were also found at higher concentrations throughout the CVM up to $6 \mathrm{hr}$ post-administration. ${ }^{12}$

Building upon this study, PLGA NPs with a variety of PEG modification densities $(2,3,5,8,10$, and $25 \mathrm{wt} \%$ ) were synthesized to study the impact of modification density on tissue 
penetration. ${ }^{34} \mathrm{NP}$ synthesis was accomplished by blending a diblock copolymer of $5 \mathrm{kDa}$ PEG and PLGA (5.6, 20, and $45 \mathrm{kDa}$ ), followed by confirmation of PEG-modification densities with H-NMR. The resulting NPs made from PEG-PLGA copolymers were tested in a murine model to assess NP diffusion and distribution within the CVM. Unmodified PLGA NPs were unable to fully penetrate vaginal rugae in the outermost epithelial layer, and remained sequestered in the outer mucus layer. Similarly, PEG surface densities below $10 \%$ were inefficient in penetrating the outer mucus layer. However, high surface coating densities spanning 10-25\% effectively diffused through the outer mucus layer and distributed evenly throughout the rugae. Moreover, PEG-PLGA copolymer NPs with 25\% PEG surface density exhibited significantly more surface coverage than all other NP formulations. ${ }^{34}$

Comparing these results with previous in vitro work, where it was observed that PLGA NPs partially coated (10 wt $\%)$ with $5 \mathrm{kDa}$ PEG achieved enhanced diffusion relative to unmodified PLGA NPs,${ }^{14}$ a coating density of $10-25 \%$ was needed to achieve improved CVM diffusion in this in vivo study.$^{34}$ This difference suggests that the minimal surface coating density required for diffusion within the CVM and uniform coverage of vaginal tissue may be underestimated by in vitro studies.

To further characterize the impact of NP surface modifications on CVM transport in vivo, PS NPs were modified with various molecular weight PEG molecules, ranging from 5 to $40 \mathrm{kDa}$, and surface modification densities $\left(1.3-3\right.$; area covered by PEG/total surface area) ${ }^{26,29} \mathrm{NPs}$ densely coated with $40 \mathrm{kDa}$ PEG exhibited the most effective transport and distribution through CVM (5-10 min post-administration). Densely modified NPs were shown to more uniformly cover the epithelium, independent of PEG molecular weight, suggesting that high molecular weight PEG can improve NP penetration and distribution through human CVM. These results 
confirm previous in vitro work, ${ }^{13,16,18,22,23,29-31,34,35,37,79}$ that ligand molecular weight and surface density play key roles in tailoring the mucoadhesive/muco-inert properties of NPs.

In a separate in vivo study, the effect of coating PCL (14.8 kDa) NPs with a tribock polymer of poly(ethylene oxide) (PEO) and poly(propylene oxide) (PPO) (PEO-PPO-PEO), relative to the typically assessed low molecular weight PEG (2-10 kDa) was evaluated. ${ }^{15}$ In female ICR mice, PEO-PCL NPs were localized in vaginal and uterine tissues $2 \mathrm{hr}$ post-administration, penetrating as much as $40 \mu \mathrm{m}$ through the vaginal tissue and distributing throughout the rugae. When comparing the diffusion coefficients of these PEO-PCL NPs (high MW PEO) to previously studied PEG-modified PS NPs (2 kDa PEG), ${ }^{22}$ the PEO-PCL NPs were only 3-7 times slower, suggesting that in spite of increased PEG molecular weight, the PEO-modified NPs adequately traversed the CVM and penetrated the vaginal epithelium.

\section{b. EVALUATION OF TRANSPORT OF UNMODIFIED NPS}

In parallel with in vitro, ex vivo, and in vivo studies that have demonstrated enhanced delivery of surface-modified, relative to unmodified NPs, recent work has shown that penetration and retention of polymeric NPs may still be attained with unmodified NPs. The transport and efficacy of unmodified siRNA PLGA NPs were assessed within the FRT by intravaginally administrating sub-200 $\mathrm{nm}$ diameter fluorescently-labeled NPs to female ICR mice. ${ }^{33} \mathrm{NP}$ distribution was assessed over 7 days, and the analysis of tissue cross-sections revealed regions of the vaginal tract in which NPs penetrated $75 \mu \mathrm{m}$ beneath the lumen. Furthermore, time-course studies using multiphoton microscopy revealed that NPs had penetrated up to $120 \mu \mathrm{m}$ below the luminal surface and were retained as long as 7 days post-treatment. ${ }^{33}$ These results suggest that unmodified NPs penetrated the CVM and distributed throughout the vaginal tract to deliver a sufficient therapeutic payload. Although NP penetration and retention were observed to induce 
therapeutic efficacy, the transport of surface-modified NP groups was not measured in comparison. The question whether surface modification improves penetration and efficacy relative to unmodified PLGA NPs requires further investigation.

\section{c. EFFECTS OF SURFACTANT ON IN VIVO TRANSPORT}

Concurrent with the described work that has correlated surface modification to diffusion and distribution in the FRT, other studies have explored the effect of utilizing a biocompatible surfactant, pluronic F127, to produce mucus penetrating particles. ${ }^{36}$ To investigate the improved chemotherapeutic efficacy obtained from these particles, a commonly used chemotherapeutic, paclitaxel, was encapsulated in the F127-modified NPs. When administered intravaginally in TC-1 tumor bearing mice, unmodified NPs aggregated within the vaginal lumen, preventing diffusion to the underlying epithelium, even after $20 \mathrm{hr}$. In contrast, NPs coated with F127 demonstrated significantly improved distribution and diffusion, uniformly dispersing through the CVM and penetrating to the underlying epithelial tissue. This enhanced diffusion translated to improved therapeutic efficacy, with F127-modified NPs reducing tumor growth by approximately $93 \%$ relative to the untreated control group, while unmodified NPs reduced the tumor load by only 42\%. Moreover, survival time was extended to 11 and 19 days, for unmodified and surface-modified NPs respectively, relative to a survival time of 9 days for the

untreated mice. ${ }^{36}$ This study highlights the use of an unconventional surface modification to increase the chemotherapeutic efficacy of intravaginally administered drugs.

\section{d. EFFECTS OF MUCUS PROPERTIES AND ADMINISTRATION SOLUTION ON IN}

VIVO TRANSPORT 
While many of these in vivo (and in vitro/ex vivo) studies have focused on surface ligands and surfactants to enhance NP distribution, other methods have assessed the role of mucus properties to provide enhanced NP delivery to the FRT. Since both PEGylated PS and PLGA NPs have shown varying degrees of efficacy over a range of PEG molecular weight and surface densities, a murine model was used to study the conditions in which mucus-penetrating PEG NPs may be delivered most effectively. ${ }^{21}$ In this study, PS and PLGA NPs modified with 2 kDa PEG were administered intravaginally in hypotonic media, mimicking advective transport. To quantify the distribution of these NPs in vivo, vaginal and ectocervical tissues were excised and imaged via fluorescence microscopy. Both PEG-modified PS and PLGA NP groups, administered in hypotonic media, were shown to uniformly accumulate and coat the entire vaginal epithelium after only 10 min. Furthermore, similarly administered surface-modified NPs, penetrated more than $100 \mu \mathrm{m}$ into the CVM within $10 \mathrm{~min}$. As a function of surface-modification, images revealed that $88 \%$ and $86 \%$ of the tissue surface was covered with PEG-modified NPs, while only $30 \%$ and $36 \%$ coverage was attained with unmodified NPs in vaginal and ectocervical tissue respectively. ${ }^{21}$ These studies corroborate other in vitro and in vivo data to suggest the significant impact PEGylation has on penetration/distribution kinetics in CVM, and provide insight into the impact of administration method and conditions on NP diffusion. Exploiting the more rapid fluid influx of NPs to the vaginal epithelium, observed using hypotonic conditions, may provide an effective administration technique that relies on advective transport rather than diffusion alone.

Subsequent work, ${ }^{88}$ building upon ${ }^{21}$, investigated the impact of fluid osmolality by utilizing modest to high hypotonic solutions to administer PEG-modified (5 kDa) PS NPs to the murine 
vaginal tract. ${ }^{88}$ When delivered in both high and low hypotonic solutions $(20$ and $220 \mathrm{mOsm} / \mathrm{kg}$, respectively) PEG NPs were shown to rapidly penetrate the CVM and vaginal lumen, covering $88 \%$ and $76 \%$ of the underlying vaginal epithelium. This was a significant increase relative to the isotonic solution $(294 \mathrm{mOsm} / \mathrm{kg}$ ), in which PEG NPs only covered $60 \%$ of the vaginal epithelium. In addition to enhanced distribution and penetration, PEG-modified NPs exhibited higher vaginal retention $1 \mathrm{hr}$ post-treatment when delivered in a hypotonic solution. In nonambulatory mice, $69 \%$ and $83 \%$ of NPs were retained for isotonic and hypotonic solutions, respectively, while for ambulatory mice, the retention rate for the isotonic solution was $22 \%$, and remained somewhat constant $(75 \%)$ for the hypotonic solution. In both cases, PEG NPs administered in a hypotonic solution were retained within the vaginal tract for longer durations, indicating that the rapid delivery of PEG NPs to the vaginal surface using a hypotonic solution promotes NP retention. ${ }^{88}$ These results confirm the enhanced distribution and penetration effects from using hypotonic solutions to administer NPs intravaginally. They also underscore that administration solution, in addition to NP surface-modification, may have a significant impact on in vivo mucus properties, impacting drug delivery and distribution through the CVM.

\section{e. EFFECTS OF POLYMER TYPE ON IN VIVO TRANSPORT}

In addition to assessing surface-modifications and delivery conditions, different polymeric delivery vehicles have been evaluated in murine models. ${ }^{25}$ A novel formulation, comprised of phenylboronic NPs, was studied to improve NP mucoadhesion within the FRT. ${ }^{25}$ Due to the affinity of phenylboronic acid to form stable cyclic ester bonds with mucin, phenylboronic acid rich-NPs (PBNPs) were seen as an attractive option to formulate mucoadhesive delivery vehicles. Moreover, it was believed that modifying PBNPs with sodium sulfate would increase colloidal stability and form stable bonds with mucin fibers. In preliminary in vitro studies, 
PBNPs surface-modified with sodium sulfate at 5 and $10 \mathrm{wt} \%$ (PBNP-S5 and -S10) exhibited increased mucin adsorption at vaginal $\mathrm{pH}$ relative to unmodified PBNPs. ${ }^{25}$ Following intravaginal administration in mice, both PBNPs and PBNP-S5 had increased vaginal retention up to $48 \mathrm{hr}$ post-treatment, whereas the amount of control solution significantly decreased between 0 and $6 \mathrm{hr}$ post-treatment. While the in vitro and in vivo results show nuanced differences in mucoadhesion, these studies highlight the similar relationships that may govern NP distribution with different polymers. Moreover, these results confirm that differences observed within carefully controlled in vitro experiments may be less impactful in an in vivo environment where multiple factors govern distribution.

\section{f. EFFECTS OF THE REPRODUCTIVE ENVIRONMENT ON IN VIVO TRANSPORT}

While the focus of this chapter has been to convey design factors that can affect NP distribution and transport through the CVM in the FRT, it is also important to note that natural physiological changes in the CVM may affect NP diffusion. When translated to clinical application, a variety of differences exist in the human reproductive environment, contributing to increased variation of the FRT characteristics between patients. For example, age, hormone levels, and menstruation cycle are known to have a significant impact on CVM rheology and thickness, as well as the rate of vaginal fluid clearance. ${ }^{3,4,42}$ To explore the impact of menstrual cycle on NP distribution, an ex vivo experiment was conducted using CVM that had been excised from estrus phase mice or from mice that had been pre-treated with a progestin to mimic the diestrus phase of thicker CVM secretions. ${ }^{78}$ Using this model, the diffusivities of PS NPs surface modified with PEG (2 or 5 $\mathrm{kDa}$ ) were assessed, highlighting the variation in NP diffusion as a function of estrous cycle. In estrus phase vaginal tissue, 70\% of PEG-NPs exhibited higher effective diffusivities through the CVM relative to all NP formulations administered to diestrus phase mice. Furthermore, PEG- 
NPs in diestrus phase CVM had a MSD of at least 30-fold less than the same NPs in estrus phase CVM. From the lower effective diffusivities of PEG-NPs in diestrus phase CVM, it was deduced that NP movement is primarily due to thermal fluctuations of the mucus, and that NPs were likely trapped within the mucus mesh as a result of steric hindrance. ${ }^{78}$

\section{g. SUMMARY OF IN VIVO STUDIES}

The reviewed in vivo studies highlight the complexities associated with the rational design of intravaginal NP delivery systems. Key polymeric NP parameters that have been evaluated in controlled in vitro conditions include surface modifying agent, surface coating density, size, and surface charge. When evaluating NP design in the FRT in vivo, these parameters must also include variations between menstrual cycle phases, method of administration, vaginal fluid clearance and NP retention, homogeneous distribution throughout vaginal tissue, and penetration of NPs to the vaginal lumen and rugae. The information obtained from in vivo transport studies provides for a more detailed understanding of the impact that more realistic and varied conditions present in the FRT, have on NP transport and efficacy through the CVM. Moreover these studies enable more complex correlations that identify factors that differ between benchtop, and translatable systems, to identify factors that have the most significant effect attaining distribution and resulting efficacy in the FRT NP transport and efficacy through the CVM.

\section{MATHEMATICAL MODELING OF SMALL MOLECULE DIFFUSION WITHIN}

\section{THE FRT}

Mathematical modeling has been applied in the field of intravaginal drug delivery to gain insight into how FRT physiology impacts drug diffusion, transport, and dosage regimens. Mathematical models provide a means to efficiently test drug and molecule diffusion properties, simulating experimental conditions that might otherwise be challenging, labor-intensive, and inefficient to 
replicate at the bench. Yet to generate meaningful outputs from such models, the functional relationships must reflect the associated biology and the input parameters must be accurate. In particular, key input parameters such as diffusion and drug or delivery vehicle partition coefficients, necessary to model transport through the CVM, are typically derived and validated from experimental analysis.

\section{i. Application of mathematical modeling}

The modeling of drug diffusion within the FRT has historically focused on drug flow and distribution along the vaginal canal. ${ }^{3,5,42,52,89}$ Recently, mathematical modeling has been applied to assess molecule transport and distribution through the surrounding vaginal tissue and mucosa. $5,42,45,71,81,90$ Even more recently, with the advent of drug delivery vehicles and microbicides, has the value of mathematical modeling for reproductive applications expanded. The development of microbicides for prophylactic and therapeutic approaches has seen significant growth in terms of developing drug delivery platforms to improve intravaginal delivery against sexually transmitted infections. ${ }^{11,17}$ Some of these platforms, which are topically applied to the vagina, including intravaginal rings, films, gels and most recently fiber meshes, have the capacity to provide tunable release of therapeutic agents that diffuse through the vaginal epithelium to the underlying stroma. In the near future, it is expected that mathematical modeling will also provide insight into NP-mediated drug delivery through the CVM.

\section{ii. Modeling of drug molecule diffusion}

Pioneering studies developed various mass transport equations that describe the PK and PD data of small drug molecules diffusing through the CVM after topical application (Table 2). Studies by Katz et al. explored mathematical modeling as a tool to better elucidate the parameters 
governing intravaginal drug delivery. The group developed a multi-compartmental model to study the PK and PD behavior and diffusion of the antiretroviral tenofovir in the CVM as it released from an intravaginal gel. ${ }^{5}$ A series of coupled, partial differential equations characterized the transport of tenofovir through each layer of the mucosa (Table 2, Eqn. 1). The model incorporated equations to evaluate the subsequent uptake and clearance of tenofovir into the vascular and lymphatic systems once it penetrated through the mucosa. Initial studies conducted with this model focused on optimization to fit experimental PK data, and the predictive model outputs were found comparable to empirical data. From this analysis, the effect of differing biologic conditions between the cervicovaginal tissue layers, as well as the time required to achieve efficacious levels of therapeutic payload, was evaluated and confirmed when compared to concentrations measured from vaginal tissue biopsies. ${ }^{5}$

This multi-compartmental model was extended in ${ }^{91}$, to account for natural variations in the vaginal canal, and to include convective drug transport due to spreading of the application gel (Table 2, Eqn. 3). A subsequent study conducted a global sensitivity analysis on this model using Sobol indices to further understand how variations in parameters, which describe the application gel and the physiologic environment, cause disturbances in the model output. ${ }^{90}$ From this analysis, it was observed that the model output was most sensitive to the parameters characterizing the initial drug concentration in the application gel, the partition coefficient of tenofovir in the epithelium, and the rate constant at which the gel was diluted due to vaginal fluid. Very recently, a pair of coupled mathematical models building upon the work of 5,92 simulated the release of tenofovir and tenofovir disoproxil fumarate (TDF), which are structurally similar molecules, from PLGA electrospun fibers in the FRT (Table 2, Eqn. 2). ${ }^{93}$ The 
results indicate that factors such as antiviral diffusivity, mesh thickness, fiber diameter, and geometry can be simulated to create an accurate model that distinguishes the very different release profiles of tenofovir and TDF observed in vaginal tissue.

A comparative study was conducted by Katz et al. ${ }^{3}$ to highlight the fundamental mass transport principles and applications related to the diffusion and convection of drugs and small molecules through the vaginal environment (Table 2, Eqn. 1)..$^{3,82,89,91}$ The work focused on the use of models to investigate the PK and PD of tenofovir, and its associated derivative, TDF, when topically delivered to the vagina using either a gel ${ }^{3,91}$ or intravaginal ring, ${ }^{3,89}$ with differing dosage regimens. The model showed that the use of intravaginal rings created an enhanced accumulation of activated TDF in the stromal compartment that was maintained for a prolonged duration of weeks to months. Conversely, gels achieved an equivalent concentration of active agent in the stroma in less than one day; however, it was necessary to apply the gel repeatedly to maintain this concentration for longer than $24 \mathrm{hr}$. This study demonstrated how a model can be utilized to characterize the transport behavior of small molecules and, furthermore, to identify efficacious delivery modalities for specific antiviral agents. ${ }^{3}$ 
Table 2. Summary of mass transport equations used to model molecule diffusion in the FRT.

\begin{tabular}{|c|c|c|c|}
\hline & Reference & Model & Model Description \\
\hline 1 & $\begin{array}{c}\text { Katz et } \\
\text { al. }{ }^{3,5,82,90}\end{array}$ & $\begin{array}{c}\frac{\partial C_{G}}{\partial t}=D_{G} \frac{\partial^{2} C_{G}}{\partial x^{2}}-K_{D} C_{G} \\
\frac{\partial C_{E}}{\partial t}=D_{E} \frac{\partial^{2} C_{E}}{\partial x^{2}} \\
\frac{\partial C_{S}}{\partial t}=D_{S} \frac{\partial^{2} C_{S}}{\partial x^{2}}-K_{B} C_{S}\end{array}$ & $\begin{array}{l}\text { Multi- } \\
\text { Compartmental } \\
\text { Model for the } \\
\text { Diffusion of } \\
\text { Tenofovir } \\
\text { (Matlab) }\end{array}$ \\
\hline 2 & $\begin{array}{c}\text { Halwes et } \\
\text { al. }{ }^{93}\end{array}$ & $\begin{array}{c}\frac{\partial C}{\partial t}=\nabla \cdot\left(D_{E} \nabla C\right) \\
\frac{\partial C}{\partial t}=\nabla \cdot\left(D_{S} \nabla C\right)-k_{L} C\end{array}$ & $\begin{array}{c}\text { Multi- } \\
\text { Compartmental } \\
\text { Model for the } \\
\text { Diffusion of TDF } \\
\text { (Matlab) } \\
\end{array}$ \\
\hline 3 & $\begin{array}{l}\text { Gao et } \\
\text { al. }{ }^{91}\end{array}$ & $\begin{aligned} \frac{\partial C_{E}}{\partial t}=D_{E}\left(\frac{\partial^{2} C_{E}}{\partial x^{2}}\right. & \left.+\frac{\partial^{2} C_{E}}{\partial y^{2}}\right)-K_{o n}\left\{C_{E} \emptyset_{E}-\frac{C_{D P}}{r}\right\} \\
& +k_{o f f} C_{D P} \\
\frac{\partial C_{S}}{\partial t}=D_{S}\left(\frac{\partial^{2} C_{S}}{\partial x^{2}}\right. & \left.+\frac{\partial^{2} C_{S}}{\partial y^{2}}\right)-K_{B} K_{S} \\
& -K_{o n}\left\{C_{S} \emptyset_{S}-\frac{C_{D P}}{r}\right\}+k_{o f f} C_{D P}\end{aligned}$ & $\begin{array}{c}\text { Convective Drug } \\
\text { Transport } \\
\text { (Matlab) }\end{array}$ \\
\hline 4 & Lai et al. ${ }^{45}$ & $\frac{\partial \vec{V}}{\partial t}=D \frac{\partial^{2} \vec{V}}{\partial z^{2}} \pm \vec{f}(\vec{V}(z, t)) \pm \vec{g}(\vec{V}(z, t))$ & $\begin{array}{l}\text { Smoluchowski } \\
\text { Encounter Rate } \\
\text { (not disclosed) }\end{array}$ \\
\hline 5 & Lai et al. ${ }^{81}$ & $\frac{\partial u}{\partial t}=D_{A b 0} \frac{\partial^{2} u}{\partial z^{2}}$ & $\begin{array}{l}\text { Stochastic Viral } \\
\text { Diffusion Model } \\
\text { (not disclosed) }\end{array}$ \\
\hline
\end{tabular}

\section{iii. $\quad$ Modeling of diffusion of antibodies}

Mathematical models have also been used to study the transport and diffusivity of biological molecules, such as antibodies, through the CVM. Antibodies have recently emerged as a prophylactic approach to combat viral infection within the FRT. In conventional therapy, antibodies have been utilized to elicit a targeted immunogenic response. ${ }^{81}$ However, antibodies can provide an additional means to prevent viral infection. Antibodies can reduce the diffusive flux of a given virus, such as HIV, by binding to receptors on the individual virions and to mucin 
fibers within the CVM. ${ }^{45,71,81}$ This coupled binding has provided a virus trapping system, in which virus diffusion is hindered, prior to reaching the vaginal stroma. ${ }^{81}$

Studies conducted by Lai et al. have complemented experimental work, by utilizing deterministic mathematical models to characterize virus immobilization and to gain insight into the most effective parameters that reduce virus diffusion (Table 2, Eqn. 5) ${ }^{81}$ The group developed a mathematical model to study the impact that certain parameters, such as the rate of mucin binding and affinity for virion binding, have on prophylaxis. Immunoglobulin G (IgG) was chosen due to its proven efficacy in saturating the CVM and hindering HIV diffusive flux. The model results indicate that to maximize virus immobilization and minimize viral diffusive flux and thus infection, IgG antibodies must exhibit a high affinity for virion binding and a weak affinity for mucin binding.

In another study, this group applied a mathematical model to evaluate the potential effects of secretory immunoglobulin $\mathrm{A}(\mathrm{IgA})$ in the CVM as a means to agglutinate and prevent $\mathrm{HIV}$ transmission (Table 2, Eqn. 4). ${ }^{45}$ The model investigated the mechanisms by which secretory IgA binds to HIV, causing agglutination and aggregation and thus preventing infection. Experimental data were used to simulate virion collision kinetics in a physiologically accurate environment. Outputs from the model suggest that, regardless of viral load, secretory IgA-related agglutination is most likely not the primary mechanism by which viral and pathogenic infection is mediated. Furthermore, the model implies that this agglutination phenomenon is most likely effective against pathogens where diffusion is characterized as other than Brownian motion or has a high propensity for collision and encounter rates. ${ }^{45}$ 


\section{iv. Definition of key parameters}

Expanding modeling capabilities to more complex environments requires that predictive deterministic and mechanistic models be applied and adapted to fit empirical data, such that the PK and PD properties of small molecule drug diffusion and transport may be better understood. Data gathered empirically in in vitro tissue mimics, in vivo, or ex vivo tissue models are therefore critical to extrapolate key transport parameters. In particular, when building a model to predict drug delivery within and through the CVM, whether it be small molecule release from a topically-applied platform or free drug delivery, the associated parameters must be precisely defined because the model output is dependent upon and sensitive to these parameters. Further, the geometry and assumptions made about the environment when creating a model are critical to its effectiveness. In studies where small molecule diffusion through mucus membranes has been modeled, ${ }^{3,4,42,71,89}$ it was found that the orientation of the mucin fibers as well as mucus porosity play critical roles in defining models that yield accurate simulations. The vaginal mucosa, the physiologic effects of menstruation on fluid volumes and pressures within the vaginal canal, the cell densities of the lamina propria and stromal layers, and the properties of the mucus gel itself, significantly impact diffusive transport. These characteristics need to be accurately described by the model parameters and functional relationships. In addition to having an appropriate understanding of the environment being modeled, it is also necessary to define the physicochemical and PK parameters of the therapeutic agent of interest. Further, in terms of delivery platforms, relevant parameters for fiber meshes include mesh thickness, fiber diameter, and geometry, while for NP-based transport, the characteristics of the delivery vehicle include lipophilicity, surface charge, molecular weight or average size, and diffusion coefficient. These parameters are typically measured and extrapolated from empirical data, as illustrated in ${ }^{5,91}$. 


\section{v. Summary of modeling of small molecule transport through the FRT}

Although the mathematical modeling of small therapeutic molecules in the FRT, and more specifically through CVM, is in its nascent stages, substantial progress has been made to date. The modeling work has advanced the understanding of the characteristics of small molecule diffusion as well as the associated PK and PD behaviors in the complex environment of the FRT. Accounting in more detail for the nonlinear characteristics of cervical mucus as well as natural variations in the vaginal canal may be necessary to obtain further insights. Modeling different cell types and their interactions, such as stromal and immune cells, may help to fine-tune the therapeutic response. Tailoring model parameters for different drugs and therapy modalities would help to move this work toward predictive capability. In particular, more mobile delivery vehicles, such as polymeric NPs, could encapsulate and protect active agents from unwanted cellular interactions or degradation, and carry them through the mucosa. Mathematical modeling could be applied to tailor such NPs to have mucoadhesive properties, to "stick" to the mucus layer, slowly releasing drug at this location, or to utilize muco-inert features to penetrate the epithelium and release drug within the stroma. Further, the coupling of various models, such as drug release from electrospun fibers with drug diffusion within the FRT, ${ }^{93}$ holds the promise for an integrated understanding of therapeutic efficacy. Ultimately, the development of modeling frameworks that include user, therapeutic, and physiological characteristics may offer practical tools to complement current clinical approaches by informing therapeutic design that is personalized to individual patient needs. 


\section{UTILIZING EMPIRICAL STUDIES AND MATHEMATICAL MODELING AS}

\section{COMPLEMENTARY TOOLS TO INFORM NP DESIGN}

Topical drug delivery to the FRT is a burgeoning field with many applications spanning chemotherapeutic treatments to microbicidal interventions. Polymeric NPs have demonstrated promising potential for efficacious delivery to the FRT, by enhancing the diffusion of encapsulated agents through the CVM, in addition to imparting tailorable mucosal interactions. As has been addressed in this chapter, several experimental approaches - comprised of in vitro, ex vivo, and in vivo systems - have been utilized to characterize NP delivery and transport through the vaginal mucosa. From these studies, it can be appreciated that NP-CVM interactions are complex, and that the parameters governing these interactions must be considered to rationally design efficacious NP delivery systems.

Several factors may enhance or impede NP diffusion in the CVM. The in vitro and ex vivo studies discussed highlight that NP diffusion through the mucosa is dependent on ligand choice, modification density, and molecular weight. Expanding upon these studies, in vivo experiments have provided more complex environments in which to assess NP transport and diffusion. In addition, in vivo studies have shown that NP transport varies as a function of the administration method (here tonicity and advection) and menstrual cycle phase at the time of administration. Furthermore, these studies utilize a physiologically relevant environment to provide spatiotemporal details that relate NP diffusion to penetration depth and homogeneity of NP distribution within vaginal tissue. 
Despite the relationships elucidated by in vitro, ex vivo, and in vivo experiments, one of the challenges facing experimental systems is the scarce number of specimens available. The limited number of samples - whether human or synthetic mucus, ex vivo tissue, or in vivo animal models - are unable to meet the experimental needs to test the vast number of possible parameter variations involved in NP design. Even if human specimens were plentiful, iterative experiments would be inefficient to evaluate single or multiple parameter variations across every parameter of interest. Furthermore, variations exist between human CVM samples for in vitro studies, ex vivo tissue, and in vivo animal tissue, which make it difficult to translate or scale-up for seamless comparison to the human FRT. Additionally, synthetic mucus and tissue samples derived from animals lack some of the complex physiological properties that are unique to the human FRT.

Complementary to experimental models, mathematical modeling presents a platform that enables a systematic assessment of how different mechanisms and interactions affect diffusion and transport within the CVM. Mathematical models have been used to study the release characteristics of topically applied delivery systems (e.g., intravaginal rings, gels) and small molecule transport through the CVM (antibodies, antivirals). However, to our knowledge, there have been no mathematical models that investigate the impact that the mucosal diffusion of NPs has on the delivery or efficacy of therapeutic encapsulants, nor that distinguish NP diffusion from the pharmacokinetics of therapeutic payload release. Moreover, as the field expands to include more labile biological molecules (e.g., oligonucleotides, peptides, proteins), physiological conditions will have an increased impact on molecule activity as a function of release and tissue/intracellular location. Similar to drug delivery, individual biologic agent characteristics will vary as a function of environment, but may more rapidly be affected based on 
release, binding, and internalization. Furthermore, for next-generation multipurpose delivery platforms - where multiple active agents, multiple types of delivery vehicles, or both are combined - it will be beneficial to elucidate these interactions alone and in combination with predictive and validating design tools.

Although mathematical modeling faces difficult challenges, such as maintaining biological relevance and minimizing computational costs, it is the primary means through which complex system analysis can be performed to gain further insight into in vitro, ex vivo, and in vivo data. Models can be built and tailored to integrate the knowledge acquired from experimental data and to enable the effective assessment of variation in system parameters. Furthermore, while current experimental techniques are often limited to evaluating one, or at most two different parameters in one study, modeling provides a high-throughput means to systematically evaluate multiple parameters.

Given the complexity of the FRT environment, more comprehensive models may be required to accurately simulate vehicle parameters to effectively model NP transport. In particular, a model that integrates ligand density, molecular weight, and hydrodynamic NP size would be beneficial to account for variation in diffusion through the different layers and tissue types of the FRT. Furthermore, ligand (e.g., molecular weight, charge, hydrophobicity, affinity) and polymer types used to fabricate delivery vehicles may be characterized and included in the model framework to highlight how these molecules interact with the mucosa. For instance, relationships between characteristics such as surface charge, hydro- or lipophilicity, propensity or rate of mucin binding, and self-aggregation, may be of interest, as these have been observed to affect diffusive 
transport. Modeling these NP properties in relation to their mucosal interactions would result in a more comprehensive model and, thus, more translatable and insightful simulations.

As highlighted by some of the in vivo work summarized here, and in addition to the properties inherent to NP formulation and characterization, the method of NP administration also impacts transport. The in vivo studies have shown that the tonicity and osmolarity of the topically-applied NP suspension have a significant impact on NP penetration and transport in the mucosa and vaginal tissue. More specifically, the tonicity and osmolarity dictate the predominant method of transport - usually a combination of bulk fluid flow and diffusion - and thus the rate of NP transport and vaginal distribution. Despite this impact, these parameters have only been investigated in limited studies. Incorporating administration properties and methodology into predictive mathematical models may provide more insight into the extent of interdependency between NP properties and administration method.

In addition to NP-specific characteristics and delivery vehicle administration conditions, the biological and structural properties of the mucosa itself can affect NP diffusivity, and are thus of significant interest to model. Alterations in mucin fiber arrangement and mucus viscosity are related to stage of menstrual cycle, age, and hormone levels, all of which vary from patient to patient. These patient-specific dependencies, make it difficult to characterize their effects with the limited in vitro and in vivo experimental techniques and specimens currently available. Therefore, including these parameters in a mathematical model may enable a more thorough understanding of how anatomical and physiological properties impact the fate of NP delivery systems, which has yet to be fully characterized empirically. Additionally, future work may 
focus on addressing limitations such as emulating vaginal fluid clearance and the variations in mucus composition and tissue thickness, which prove difficult to recapitulate in vitro.

The current knowledge gaps or limited experimental data may be informed by mathematical modeling. Comparisons across experimental model types (e.g., ex vivo murine with in vivo murine with human clinical trials) may be made, and explored in greater depth via mathematical models that are used to collect, retain, and refine this information across studies. This integrative approach would more readily enable the rational design of tailored and personalized delivery systems that meet patient-specific needs. The evolution and connectivity of these models could have significant clinical impact, identifying new ways to target FRT tissue, and increase the efficacy of next-generation delivery systems.

In addition to NP characteristics, administration methods, and patient-specific features that broadly govern delivery, the studies conducted to date highlight select choices in NP design features. For example, currently, the majority of NP diffusion studies have investigated the use of muco-inert (PEG) or mucoadhesive (chitosan) surface-modifications. However, within the field of NP delivery, other modifying agents have been utilized such as cell penetrating peptides $(\mathrm{CPPs}){ }^{1,2,28}$ which have intracellular targets and promote rapid cellular internalization. As new biologic delivery needs will necessitate both transport and cell internalization, the balance between features imparted by multiple ligand types will be valuable to explore. To date, there are few studies that address how NP diffusion varies as a function of such ligand modification, with only cursory studies investigating overall surface charge. In addition to peptides that promote ubiquitous internalization, specific ligands that target epithelial or immune receptors, may be of interest for reproductive cancers or to prevent virus uptake, or to provide virus-like targeting to 
host tissue. As such, next-generation delivery vehicles may rely on combinations of different ligands, with different molecular weights and characteristics than the typically modeled PEG. Moreover, tunable surface features may enable temporal changes to carriers (e.g., pH-responsive ligand release or sheddable ligands) that change carrier transport as a function of location and time. As this type of drug delivery has applications in both cancer chemotherapy and microbicides, it would be advantageous to have an improved understanding of how these NP delivery systems transport and exert their effect within the FRT. 
II. Enhanced uptake and transport of PLGA-modified nanoparticles in cervical cancer

Adapted From:

Sims LB, Curtis L, Frieboes H, Steinbach JM; Enhanced Uptake and Transport of PLGA-modified Nanoparticles in Cervical Cancer Cells; April 22, 2016; Journal of Nanobiotechnology ${ }^{2}$

\section{A. CLINICAL SIGNIFICANCE AND BACKGROUND}

Cervical cancer is the third most common gynecologic cause of cancer associated with patient fatalities. Approximately 13,000 new cases of invasive cervical cancer are diagnosed yearly, of which $30 \%$ prove fatal. In the US, cervical cancer primarily afflicts women younger than 50; however, in countries without established screening and prevention programs, cervical cancer remains the second most common type of cancer and cause of death among all female cancers ${ }^{94-}$ 96. Screening tests and vaccines have contributed to a decrease in cases; to date, there are 3 approved vaccines against cervical cancer. These vaccines, Gardasil@, Gardasil@ 9,

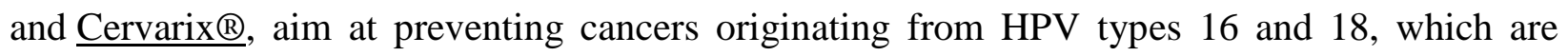
attributed to $\sim 70 \%$ of cervical cancers ${ }^{97}$. Yet despite this efficacy, the vaccines only protect against a subset of all known HPV strains ${ }^{97-99}$. The inadequacy of vaccination, coupled with the fact that vaccination is not widespread ${ }^{98,99}$, maintains the risk of cervical cancer as a fatal disease.

Relative to the success of prophylactic vaccines, ineffective treatment options exist for established HPV infections and cervical cancer originating from HPV. This is primarily attributed to multidrug resistance and chemotherapeutic side effects. Despite early stage tumor identification and established eradication methods including surgery and radiation, the adverse side effects of these treatment strategies often result in negative gynecologic and obstetric 
outcomes. In comparison to surgical and radiation challenges, even systemic chemotherapy results in relatively low transport efficiency, resulting in high chemotherapeutic doses needed to target mucosal and epithelial tissue ${ }^{36,100-102}$. Due to the high systemic toxicity induced by systemic chemotherapy, new treatment strategies are urgently needed.

In addition to these clinical challenges, successful therapeutic agent delivery to the tumor microenvironment requires minimizing agent degradation and excretion, avoidance of immunogenic interactions, adequate penetration and distribution throughout the tumor tissue, cellular uptake and internalization, and sufficient cytotoxicity ${ }^{103}$. United States Food and Drug Administration (FDA)-approved materials may be selected as nano-scale drug carriers that have been proven to be non-inflammatory and non-toxic while enabling the delivery of highly localized concentrations of both hydrophilic and hydrophobic agents ${ }^{103-105}$. In particular, polymer nanoparticle-based drug delivery systems have been evaluated as attractive options for efficacious delivery of agents such as drugs and genes with resulting treatment efficacy ${ }^{104,105}$. The pharmacokinetics of polymeric systems can be tailored by changing the polymer monomer concentrations to facilitate agent diffusive transport, typically via a combination of burst and sustained release profiles.

Besides enabling the sustained release of encapsulated agents, relative to systemic options, local intravaginal therapy may represent a relatively non-invasive approach to locally treat cervical tumors ${ }^{100}$. Nanoparticles (NP) can be modified to minimize unwanted systemic interactions, prolong bioavailability, promote targeted delivery to the physiologic site of interest, and enhance tumor penetration and cellular uptake ${ }^{106}$. Different types of NP surface modifications can be utilized to elicit these desired behaviors. Stealth coatings such as polyethylene glycol (PEG) can decrease unwanted systemic interactions and neutralize the carrier's surface charge ${ }^{107,108}$, while 
targeting ligands can increase the specificity of conjugated carriers to systemically target the desired physiological environment ${ }^{109}$. Cell penetrating peptides, such as MPG, RGD, and Tat1, are examples of short ligands that can enhance the cellular internalization of their conjugated carriers ${ }^{55,110-120}$. In particular, previous studies have shown that NP surface modification with the amphipathic, synthetic peptide MPG derived from HIV gp41 and SV40 ${ }^{115,121}$ displays high cellular binding and internalization achieved via clathrin-mediated endocytotic uptake ${ }^{28}$.

Tumor tissue is typically characterized by an overabundance of extracellular matrix as well as poorly vascularized areas, both of which can hinder diffusive transport of NPs ${ }^{103}$. Thus, in addition to evaluating cellular uptake capability, delivery systems would benefit from testing in a three-dimensional (3D) environment which more closely represents hypo-vascularized tumor lesions. For this purpose, 3D cell culture provides a suitable platform ${ }^{122,123}$ that is more controllable than tumors in vivo. Previously, 3D cell culture has been employed to study the behavior of various nano-carriers, such as gold and polymeric NPs. It has been shown both experimentally 48,124-127 and theoretically 49,51,52,61,128-133 that NPs require appropriate characteristics and surface modifications to adequately penetrate hypo-vascularized regions for effective therapeutic delivery, even if cellular internalization is optimized.

With the goal to enhance both the transport and internalization of therapeutic agents in hypovascularized tumor tissue, in this study we evaluate a variety of poly(lactic-co-glycolic) acid (PLGA) NP formulations for tissue penetration and cellular internalization. The NPs were modified with either a cell penetrating peptide (MPG), a stealth ligand (PEG), a tumor targeting cell penetrating peptide (Vimentin tubulin binding site peptide, VIM) ${ }^{134,135}$, or a hybrid 
modification with both cell penetrating peptides and stealth ligands (MPG/PEG). These ligands were chosen because they have already been individually well characterized to enhance cell internalization, e.g., as we have previously shown for MPG. We assess how the NP surface modifications affect uptake in cervical tumor cells, and compare the NP performance in normal vaginal cells. We further evaluate the NP transport and internalization in a 3D spheroid model representing hypo-vascularized cervical cancer lesions.

\section{B. METHODS}

\section{Synthesis of avidin-palmitate conjugates}

Avidin-palmitate was conjugated to NP surfaces as previously described ${ }^{39,136}$ for subsequent reaction with biotinylated ligands: MPG, PEG, VIM, and an equimolar combination of MPG/PEG. Briefly, $40 \mathrm{mg}$ of avidin was dissolved in $4.8 \mathrm{~mL}$ of $2 \%$ sodium deoxycholate (NaDC) in phosphate buffered saline (PBS) warmed to $37{ }^{\circ} \mathrm{C}$. Palmitic acid-Nhydroxysuccinimide ester (PA-NHS, Sigma) was dissolved in $2 \% \mathrm{NaDC}$ at $1 \mathrm{mg} / \mathrm{mL}$ and sonicated until well-mixed. $3.2 \mathrm{~mL}$ of the $1 \mathrm{mg} / \mathrm{mL}$ PA-NHS solution was added dropwise to the reaction vial, and reacted overnight at $37{ }^{\circ} \mathrm{C}$. The following day, the reaction was dialyzed overnight in $1200 \mathrm{~mL}$ of $0.15 \% \mathrm{NaDC}$ in PBS heated to $37{ }^{\circ} \mathrm{C}$. Free PA-NHS was removed using 3500 molecular weight cut off (MWCO) dialysis tubing, and the dialysis cassette contents were subsequently transferred to a vial and stored at $4{ }^{\circ} \mathrm{C}$.

\section{Nanoparticle synthesis}

We synthesized and characterized PLGA NPs encapsulating a fluorescent dye, Coumarin 6 (C6), to evaluate tumor penetration and distribution via fluorescence microscopy. From earlier 
studies $^{33,137-139}$ as well as our previous experiments, we have observed that negligible quantities $(\sim 1 \%)$ of C6 are released from NPs. This is attributed to the hydrophobic nature of C6 encapsulated within hydrophobic NPs. Therefore, C6 detected in cells reflects NP distribution in or on the cells, not C6 release and distribution. C6 NPs were synthesized as previously described using an oil-in-water $(\mathrm{o} / \mathrm{w})$ single emulsion technique ${ }^{39}$. Briefly, C6 was encapsulated into 100200 mg PLGA carboxyl-terminated polymer (0.55-0.75 dL/g, LACTEL®). C6 was dissolved in methylene chloride (DCM) overnight at a concentration of $15 \mu \mathrm{g}$ C6 per mg of PLGA. The following day, the solution was added dropwise to a $5 \%$ polyvinyl alcohol (PVA) solution of equal volume, vortexed and sonicated. The resulting NPs were hardened during solvent evaporation for $3 \mathrm{~h}$. For unmodified NPs, the NPs were washed after hardening, and centrifuged 3 times at $4{ }^{\circ} \mathrm{C}$ in deionized water $(\mathrm{diH} 2 \mathrm{O})$ to remove residual solvent. For avidin-palmitate surface-modified NPs, a similar protocol was followed ${ }^{39,137}$. NP formulations were synthesized by adding ( $1 \mathrm{mg} / \mathrm{mL})$ avidin-palmitate to the $5 \%$ PVA solution. NPs were collected after the first wash and incubated for 30 min with biotinylated ligands at a molar ratio of 3:1 ligand:avidin in PBS. After conjugation, the NPs were washed two more times with diH2O by centrifugation and subsequent washing. All NPs were frozen, lyophilized, and stored at $-20^{\circ} \mathrm{C}$ until use.

\section{Cell culture}

VK2/E6E7 vaginal epithelial (VK2) and human cervical carcinoma (HeLa) immortalized cell lines were kindly provided by Dr. Kenneth Palmer's lab (University of Louisville). These cell lines were obtained and authenticated through ATCC. We selected the VK2/E6E7 cell line, since for intravaginal delivery these would be the first "normal" cells to encounter NP treatment. HeLa cells provide the ability to assess NP behavior against a cervical cancer cell line in vitro. VK2/E6E7 vaginal epithelial cells (VK2) were maintained in Defined Kerotinocyte-Serum Free 
medium (SFM) supplemented with Defined Keratinocyte-SFM Growth Supplement. HeLa cells were maintained in minimal essential media (MEM) supplemented with $10 \%$ fetal bovine serum and $1 \%$ penicillin-streptomycin in standard culture conditions. Cell media was checked and changed daily.

\section{Tumor spheroid formation}

HeLa cells were used for tumor spheroid formation. Cells were grown to $80 \%$ confluence before harvesting. Twenty-four well tissue culture plates (Corning) were coated with a $1 \%(w / v)$ agarose gel $24 \mathrm{~h}$ before spheroid formation to prevent cell adherence. For formation, 100,000 cells were placed in each well and lightly shaken $(100 \mathrm{rpm})$ for $15 \mathrm{~min}$ on a reciprocating shaker. After 7-14 days of incubation, spheroid formation occurred by self-aggregation to sizes ranging from 500 to $1000 \mu \mathrm{m}$.

\section{Monolayer (2D) cellular uptake and microscopy}

2D cellular imaging of 1.5 and $24 \mathrm{~h}$ NP uptake and penetration in HeLa and VK2 cells was performed via inverted epifluorescence microscopy. VK2 and HeLa cells were seeded 24 h prior to NP administration in LabTek 8-well chamber slides at a density of 50,000 and 40,000 cells per well for 1.5 and $24 \mathrm{~h}$ uptake, respectively. For NP administration, NPs were massed out and dissolved to reach a final stock concentration of $0.6 \mathrm{mg} / \mathrm{mL}$ in $\mathrm{PBS}++$ (containing $\mathrm{CaCl} 2$ and $\mathrm{MgCl} 2$ ) to aid cell adherence. One hundred microliters of fresh media were added to the cells, and NPs were then added to obtain a final NP concentration of $200 \mu \mathrm{g} / \mathrm{mL}$.

After either 1.5 or $24 \mathrm{~h}$ incubation, cells were washed five times in $0.5 \mathrm{~mL}$ of $1 \mathrm{X}$ PBS to remove any unbound or non-internalized NPs. Cells were then fixed with $0.3 \mathrm{~mL}$ of $4 \%$ paraformaldehyde and incubated for $10 \mathrm{~min}$ at room temperature (RT). Cells were subsequently 
washed twice with $0.5 \mathrm{~mL}$ of PBS and permeabilized with $0.3 \mathrm{~mL}$ of $0.1 \%$ Triton X-100 in $1 \%$ bovine serum albumin (BSA) PBS++ for $10 \mathrm{~min}$ at RT. After permeabilization, cells were incubated with $0.3 \mathrm{~mL}$ of 1:40 Texas Red Phalloidin in $1 \%$ BSA PBS++ for $20 \mathrm{~min}$ at RT for cytoskeleton staining and were subsequently washed twice with $0.5 \mathrm{~mL}$ PBS. Cells were then incubated with $0.3 \mathrm{~mL}$ of $4 \mu \mathrm{g} / \mathrm{mL}$ Hoechst in $1 \%$ BSA PBS++ for $10 \mathrm{~min}$ at $37{ }^{\circ} \mathrm{C}$ for nuclear staining. Finally, cells were washed twice in PBS and once in $\mathrm{diH} 2 \mathrm{O}$, then mounted in Vectashield non-hardening mounting medium (Vector Laboratories, VWR) and kept at $4{ }^{\circ} \mathrm{C}$ until imaged.

Inverted epifluorescence microscopy was utilized to assess cellular uptake of NPs in 2D monolayers. Briefly, cells were prepared as described above and imaged in 8-well LabTek chamber slides using the following filter settings: 4',6-diamidino-2-phenylindole (DAPI) to visualize Hoechst, green fluorescent protein (GFP) for C6, and the Texas Red channel to evaluate NP uptake in 2D. Exposure times for DAPI, GFP, and Texas Red were kept consistent throughout experiments and were as follows: DAPI at $45 \mathrm{~ms}$ (excitation/emissions: $358 / 461 \mathrm{~nm}$ ); GFP at $60 \mathrm{~ms}(593 / 615 \mathrm{~nm})$; and Texas Red at $180 \mathrm{~ms}(488 / 515 \mathrm{~nm})$.

\section{Spheroid (3D) cellular uptake and microscopy}

To assess the differences in NP uptake and distribution through hypo-vascularized tumor tissue, HeLa spheroids were incubated with $0.01 \mathrm{mg} / \mathrm{mL}$ of NPs and visualized using confocal microscopy. Diffusion profiles through the spheroids for each NP formulation were evaluated by quantifying the fluorescence intensity in z-stack images as a function of distance from the spheroid periphery. After NP administration, tumor spheroids were transferred to LabTek 8-well chamber slides for fluorescent staining and were washed five times with $0.2 \mathrm{~mL} 1 \mathrm{X}$ PBS. Tumor spheroids were subsequently incubated for $10 \mathrm{~min}$ at RT with $0.2 \mathrm{~mL} 4 \%$ paraformaldehyde for 
spheroid fixation. Following fixation, spheroids were washed twice with $0.2 \mathrm{~mL}$ of $\mathrm{PBS}$, followed by incubation with $0.2 \mathrm{~mL}$ of 1:40 Texas Red Phalloidin in 1\% BSA PBS++ for 20 min at RT for cytoskeleton staining. Spheroids were then washed twice with $0.2 \mathrm{~mL}$ PBS and incubated with $0.2 \mathrm{~mL}$ of $4 \mu \mathrm{g} / \mathrm{mL}$ Hoechst in $1 \% \mathrm{BSA} \mathrm{PBS}++$ for $10 \mathrm{~min}$ at $37^{\circ} \mathrm{C}$ for nuclear staining. Finally, spheroids were washed twice in $0.2 \mathrm{~mL}$ PBS and once in $0.2 \mathrm{~mL}$ diH2O. Spheroids were then mounted with Vectashield non-hardening mounting medium. 3D uptake and distribution of NPs through tumor spheroids was assessed using confocal microscopy. Images were processed using ImageJ by taking representative samples from tumor cross-sections $(\geq 90$ averaged profiles for each sample).

\section{Flow cytometry analysis}

Cells were plated in 6-well plates (Corning) at a density of 200,000 cells per well. Both VK2 and HeLa cell lines were incubated with NP suspensions at $0.05 \mathrm{mg} / \mathrm{mL}$ for either 1.5 or $24 \mathrm{~h}$ in a 37 ${ }^{\circ} \mathrm{C}$ humidified chamber in the appropriate medium. An unmodified NP control group was used to compare uptake relative to surface-modified NPs, as our previous work demonstrated minimal difference between unmodified and avidin-modified NP association and uptake. After incubation, the cells were washed five times with PBS++. Next, the cells were dissociated with enzyme-free cell dissociation buffer (ThermoFisher). The dissociated cells were moved to FACS tubes, centrifuged, and resuspended in a FACS buffer solution containing $1 \%$ BSA and $0.1 \%$ sodium azide. From each sample, half the cells were moved to separate FACS tubes and kept on ice until analyzed (total associated samples). The remaining cells were exposed to $0.4 \%$ trypan blue for 5 min to quench extracellular fluorescence, washed twice in FACS buffer, and kept on ice until analyzed (internalized samples) . All FACS tubes from both groups were analyzed using a BD LSRFortessa Flow Cytometer (BD Biosciences). Data were analyzed using FlowJo 
software (FlowJo Enterprise), and a minimum of 10,000 cells were analyzed per sample. For flow cytometry analysis of tumor spheroids, a similar protocol was followed using spheroids after 7 days of growth. Instead of enzyme-free cell dissociation buffer, $0.25 \%$ Trypsin-EDTA was used to fully disaggregate the spheroids before centrifugation and resuspension in FACS buffer solution.

\section{Statistical analysis}

Experiments were conducted each with a minimum sample size of $n=3$. Data were analyzed by applying Tukey's test with significance $\mathrm{p}<0.05$. Unless otherwise noted, all figure error bars represent the standard deviation of the measurements. To enable clear interpretation, statistically similar results are shown linked with an overbar in the figures of the "Results" section.

\section{RESULTS}

\section{Nanoparticle characterization}

The NP surface was first modified with avidin-palmitate, and different NP groups were created based on the addition of one of the following biotinylated ligands: MPG, MPG/PEG, PEG, or VIM, as described in the "Methods" section. Figure 1 illustrates the NPs used in this study. The average unhydrated NP diameter measured $167 \pm 50 \mathrm{~nm}$. Hydrodynamic sizes and surface charges for the different NP groups were quantified via dynamic light scattering (DLS) and zeta potential measurements, respectively (data not shown). 


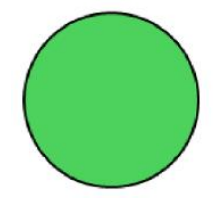

Unmodified

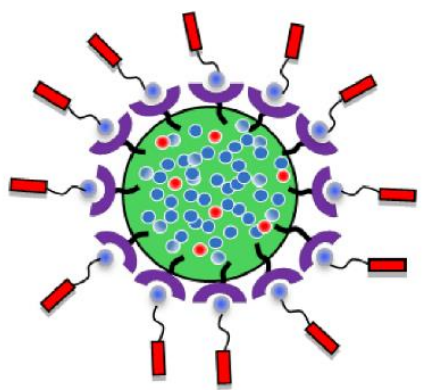

MPG, Vim, or PEG modified

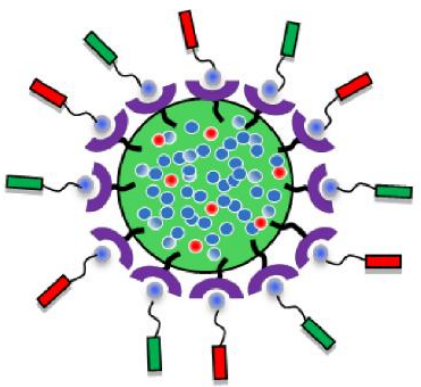

MPG/PEG (Hybrid) modified
PLGA NP

Avidin-palmitate

Biotin-peptide

Figure 1. Schematic illustrating NP formulations used in this study

Unmodified NPs had a hydrodynamic diameter of $267 \pm 13.6 \mathrm{~nm}$ and a negative surface charge of $-17.3 \pm 0.5 \mathrm{mV}$, and the addition of avidin-palmitate increased this surface charge to $-14.3 \pm$ $0.59 \mathrm{mV}$. The surface charge was further increased with the addition of the ligands, with MPGmodified NPs having the most positive charge at $-0.4 \pm 0.2 \mathrm{mV}$, followed by PEG and MPG/PEG with $-5.2 \pm 1.3$ and $-5.3 \pm 0.7 \mathrm{mV}$, respectively, and VIM with $-11.1 \pm 1.0 \mathrm{mV}$. NP hydrodynamic sizes ranged from 232 to $277 \mathrm{~nm}$ for both unmodified and surface-modified groups, with no statistically significant difference between the groups.

\section{Uptake of PLGA-modified nanoparticles in cervical cancer cell monolayers}

Cellular association (binding plus internalization) and internalization in 2D cell culture monolayers were quantitatively assessed using Fluorescence Activated Cell Sorting (FACS) with cervical tumor epithelial cells (HeLa) and normal vaginal epithelial cells (VK2) (Fig. 2). Both HeLa and VK2 cells were incubated with the same concentration of NPs $(0.05 \mathrm{mg} / \mathrm{mL})$ for 1.5 or $24 \mathrm{~h}$, regardless of modification. For HeLa cells, all of the modified NP groups showed both greater total association as well as internalization relative to unmodified NPs at both time points. 
At 1.5 h, MPG, MPG/PEG, PEG, and VIM NPs were internalized at 607×, 184×, 79×, and 57× that of unmodified NPs, respectively (Fig. 2a). Comparing the formulations to each other, the MPG NPs showed 3.3×, 7.6×, and 10.5× greater internalization than the MPG/PEG, PEG, and VIM NPs, respectively. After $24 \mathrm{~h}$ of incubation, MPG, MPG/PEG, PEG, and VIM NPs were internalized at $66 \times, 24 \times, 30 \times$, and $15 \times$ that of unmodified NPs, respectively (Fig. 2b). The MPG NPs were internalized at $2.7 \times, 2.2 \times$, and $4.5 \times$ that of MPG/PEG, PEG, and VIM NPs, respectively. Since the internalization for the MPG NPs was essentially the same at both time points, these data highlight the increase in internalization of the other formulations after longer exposure times.

\section{Uptake of PLGA-modified nanoparticles in vaginal epithelial cell monolayers}

In contrast, NP association with $\mathrm{VK} 2$ cells at $1.5 \mathrm{~h}$ was not as distinct between the various modified formulations (Fig. 2c), whereas PEG NPs internalized at $2.1 \times, 5.9 \times$, and $8.8 \times$ compared to MPG, MPG/PEG and VIM NPs, respectively. Comparing to unmodified NPs, the MPG, MPG/PEG, PEG, and VIM NPs internalized respectively at 20×, 7×, 43×, and 5×. After $24 \mathrm{~h}$ however, a clearer separation in association was apparent and PEG NPs were internalized at 1.9×, 2.8×, and 4.0× compared to MPG, MPG/PEG, and VIM NPs, respectively (Fig. 2d).

The internalization remained invariant when comparing MPG and PEG between the two time points, while the internalization of MPG/PEG and VIM essentially doubled. When compared to unmodified NPs after $24 \mathrm{~h}$ incubation, the MPG, MPG/PEG, PEG, and VIM internalized at 6.3×, $4.3 \times, 12.4 \times$, and $3.0 \times$ that of unmodified NPs. 
A

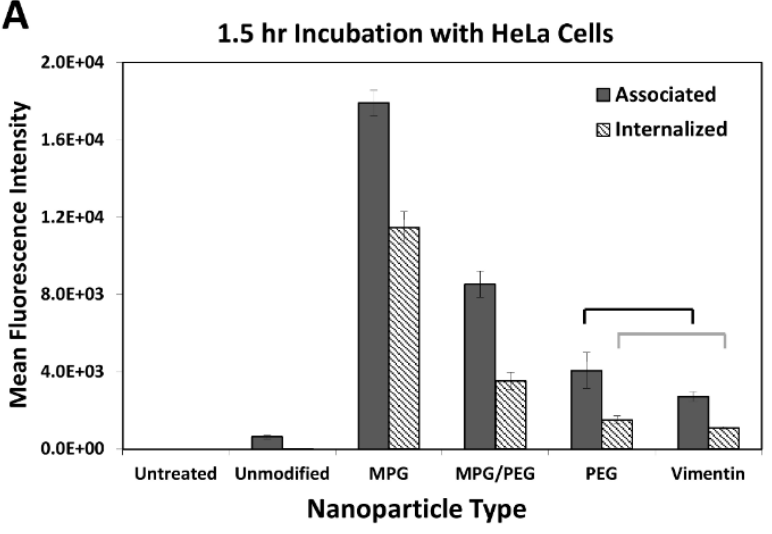

C

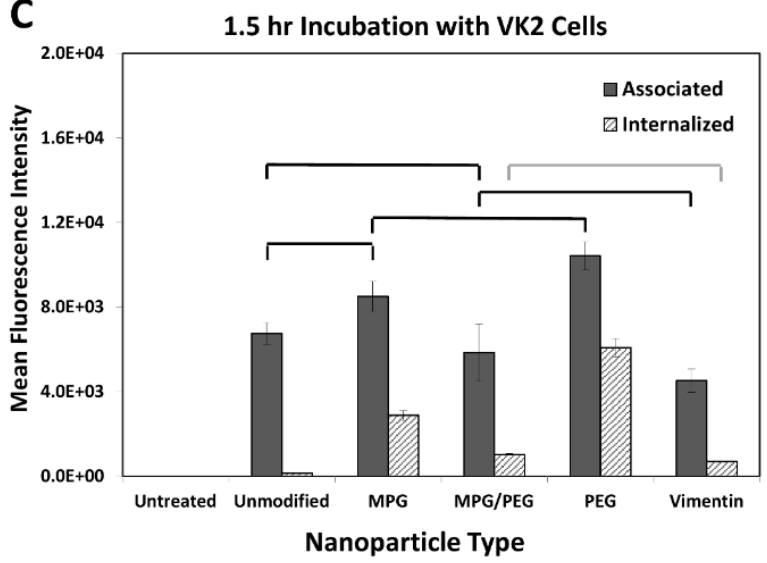

B

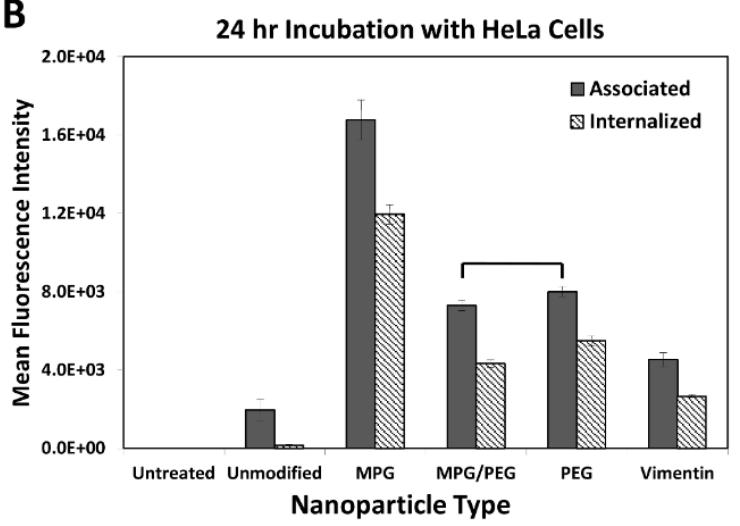

D

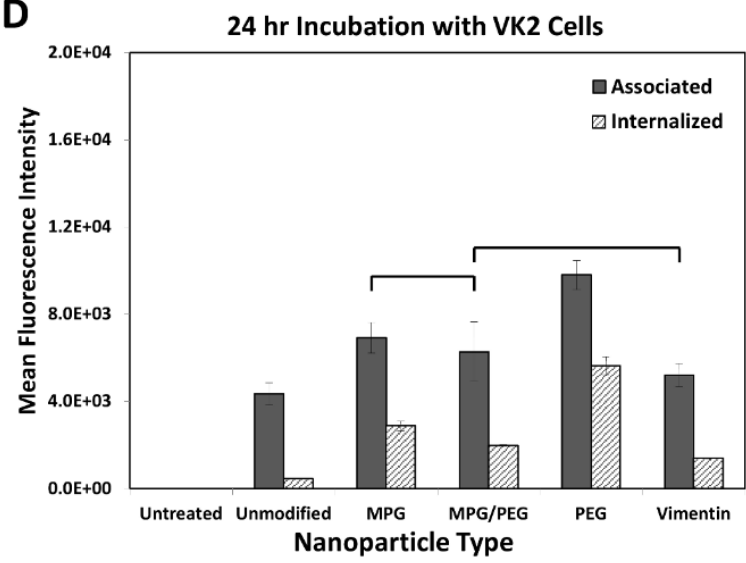

Figure 2. Cellular association and internalization of the various NP formulations are presented for HeLa (top) and VK2 (bottom) cells, shown at $1.5 \mathrm{~h}$ (left) and $24 \mathrm{~h}$ (right). Statistically similar results are shown linked with an overbar

\section{Comparison of nanoparticle uptake between tumorigenic and normal cell monolayers}

Of note after $24 \mathrm{~h}$, the unmodified NPs readily associate with the VK2 cells, in contrast to HeLa cells; however, unmodified NPs demonstrate low levels of internalization in both cell lines. In contrast, surface-modified NP uptake was significantly enhanced in both the HeLa and VK2 cell lines. MPG NPs were internalized the most in HeLa cells; whereas PEG NPs were internalized most highly in VK2 cells. While both surface-modified groups (MPG and PEG) demonstrated strong internalization in HeLa and VK2 cells respectively, MPG was internalized in HeLa cells 
$2 \times$ that of the PEG NPs by the VK2 cells. When comparing between the earlier and later time points, it is apparent that the PEG NPs showed the highest increase in both association and internalization by the longer exposure with the tumorigenic HeLa but not with the normal VK2 cells. Cellular association and internalization were qualitatively assessed via inverted epifluorescence microscopy for both HeLa and VK2 cells at $1.5 \mathrm{~h}$ (data not shown) and $24 \mathrm{~h}$ incubation times (Figs. 3, 4). Although the images for the most part reflect the flow cytometry results in Fig. IV, they are representative samples providing a limited assessment.
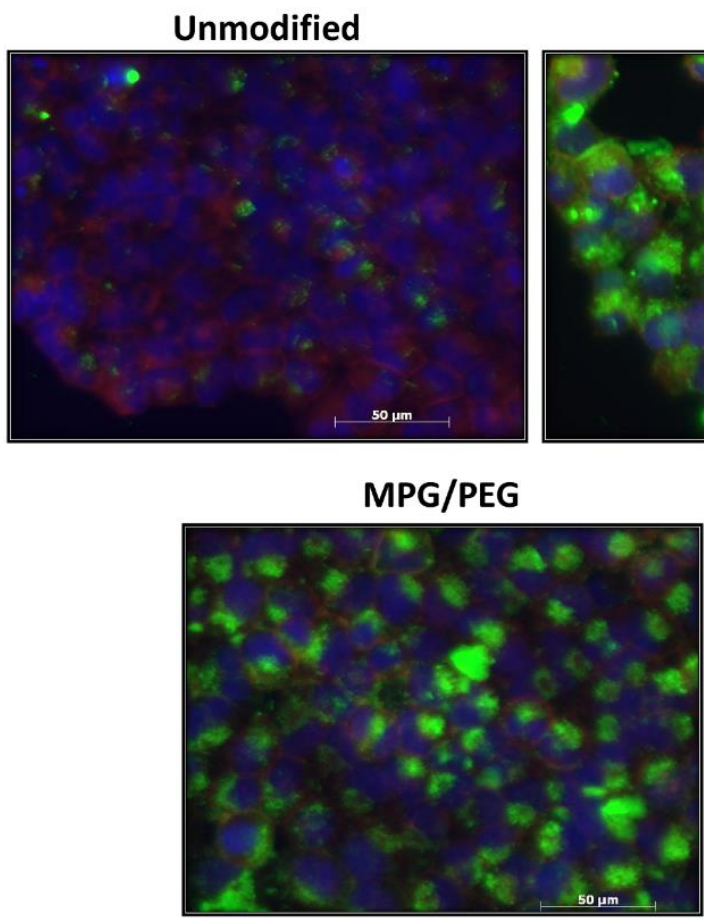

MPG

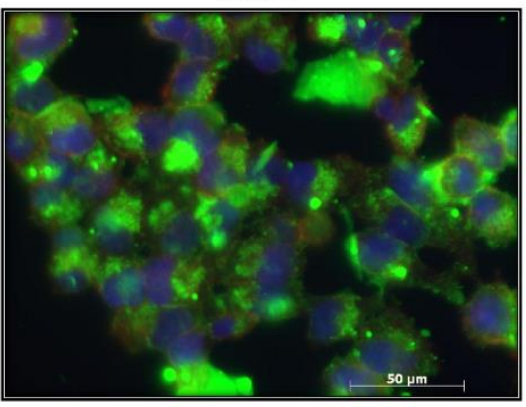

Vimentin

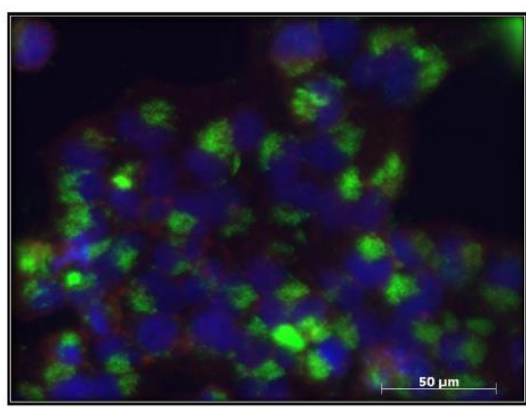

Figure 3. Total NP association (binding and internalization) in monolayers of HeLa cells after $24 \mathrm{~h}$ incubation. Nuclei are blue (Hoechst), actin cytoskeletons are red (Texas red phalloidin), and NPs are green (Coumarin 6). Bar $50 \mu \mathrm{m}$ 


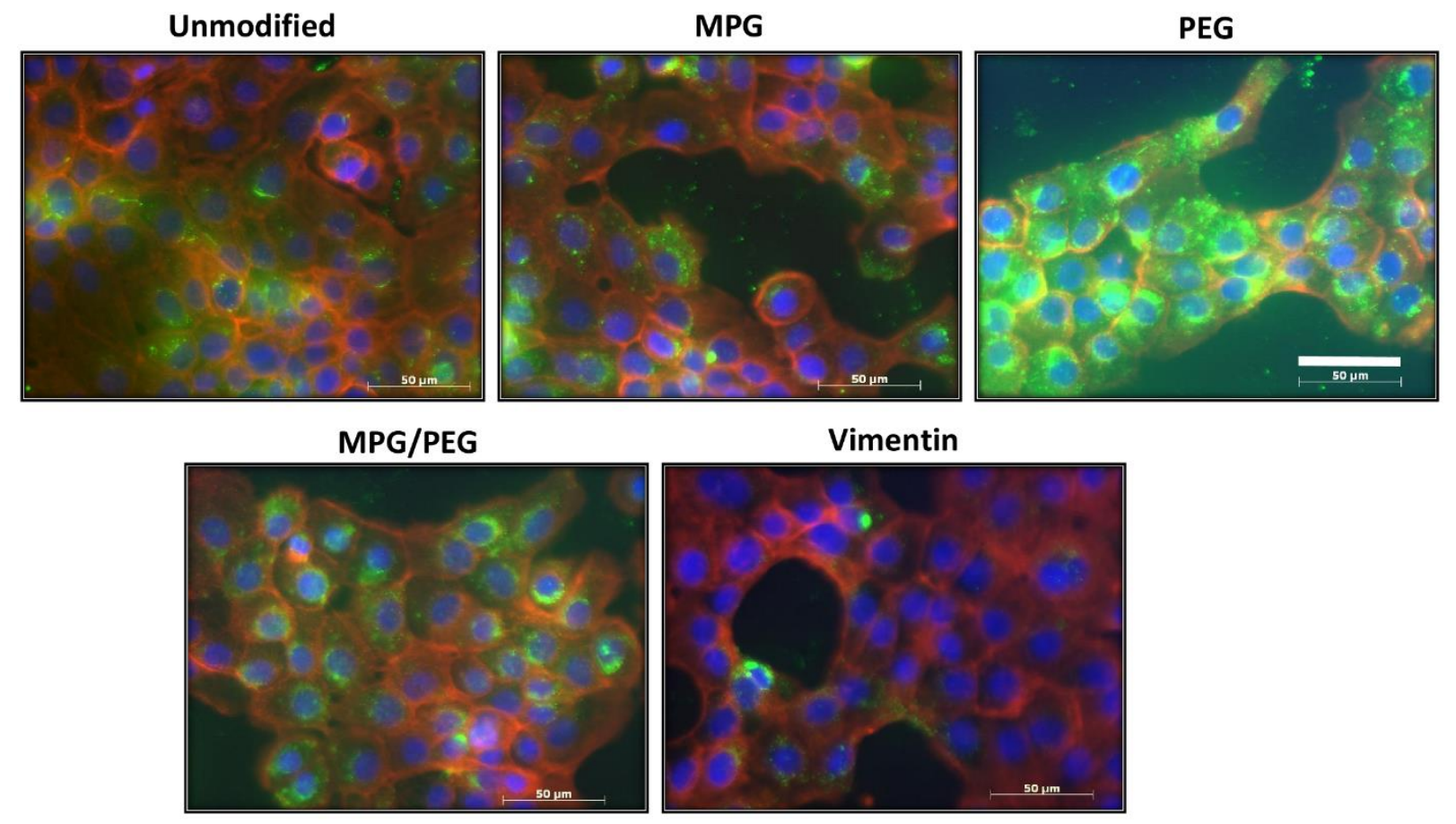

Figure 4. Total NP association (binding and internalization) in monolayers of VK2 cells after 24 $\mathrm{h}$ incubation. Nuclei are blue (Hoechst), actin cytoskeletons are red (Texas red phalloidin), and NPs are green (Coumarin 6). Bar $50 \mu \mathrm{m}$

Uptake of PLGA-modified nanoparticles in hypo-vascularized cancer lesions

Cellular association and internalization in 3D cell culture (HeLa cervical tumor spheroids), incubated with the same concentration of NPs $(0.05 \mathrm{mg} / \mathrm{mL})$ as the monolayers, were quantitatively assessed at 1.5 and $24 \mathrm{~h}$ via flow cytometry (Fig. V5). At $1.5 \mathrm{~h}$, as a group, all of the modified NPs had higher association than the unmodified NPs, with statistically similar association when compared to each other. The surface-modified NPs also evinced a statistically similar internalization when compared to each other, but in this case, as a group the MPG, MPG/PEG, and VIM NPs showed higher internalization than the unmodified and PEG NPs (Fig. 5a). In contrast, after $24 \mathrm{~h}$, the unmodified and surface-modified NPs (except for VIM, which 
was lower) showed statistically similar association (Fig. 5b), while MPG and MPG/PEG showed $2 \times$ and $3 \times$ internalization relative to PEG and VIM NPs, respectively. Interestingly, unlike the results obtained with the monolayer, in the 3D cell culture the association of all NPs increased significantly at $24 \mathrm{~h}$ compared to the $1.5 \mathrm{~h}$ time point: unmodified by $4.8 \times$, MPG by $2.6 \times$, MPG/PEG by $3.0 \times$, PEG by $1.6 \times$, and VIM by $1.6 \times$. Internalization also increased at the later time point, with MPG and MPG/PEG NPs being internalized the most (at 3.6x and 3.8x compared to $1.5 \mathrm{~h}$, respectively), while VIM or unmodified versions were internalized the least.
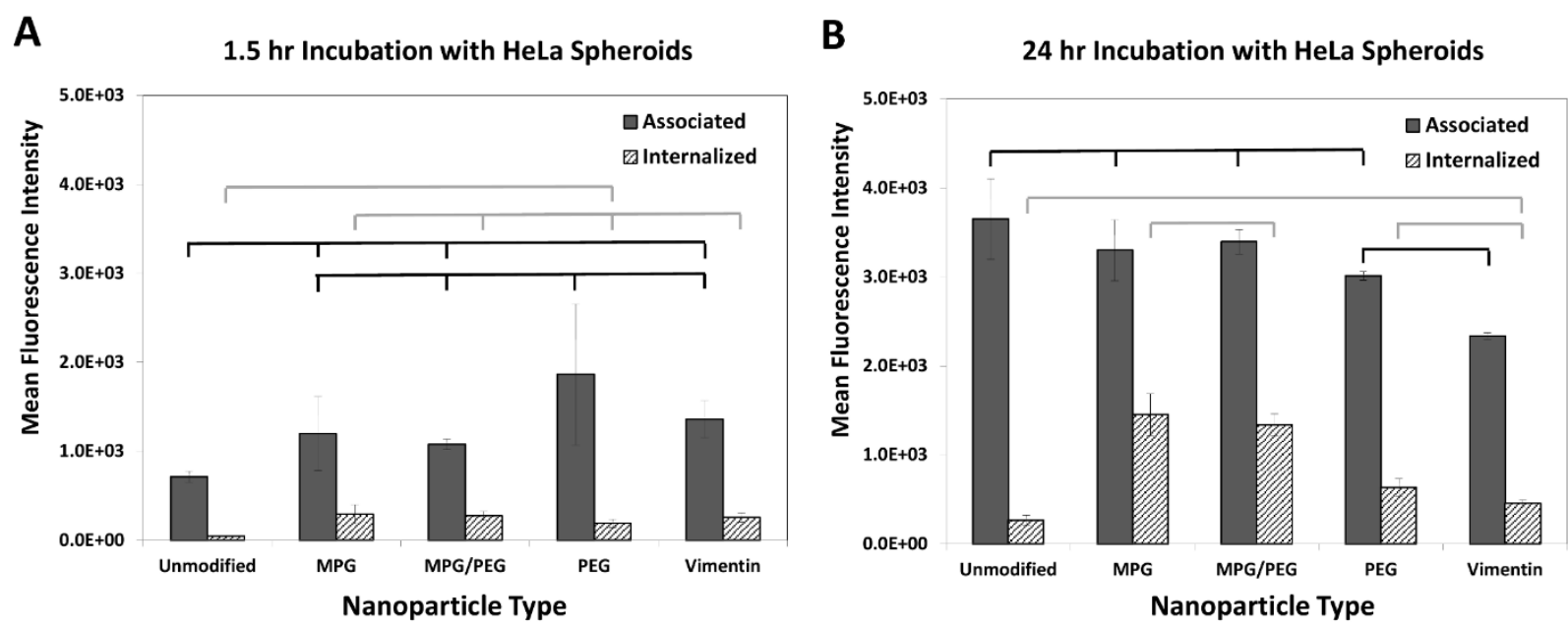

Figure 5. Cellular association and internalization of the various NP formulations in HeLa cell tumor spheroids. Results are shown at (a) 1.5 and (b) $24 \mathrm{~h}$. Statistically similar results are shown linked with an overbar Comparison of nanoparticle uptake between $2 D$ and $3 D$ cell cultures

In general, all of the values measured from 3D cell cultures were lower than those from the 2D cell cultures, highlighting the effect of the diffusive transport barrier in hypo-vascularized tissue. Figure 6 summarizes the NP cellular internalization as a function of surface modification, cell culture type, and treatment duration, highlighting the effect of diffusive transport on NP uptake 
between the monolayer and tumor environments. It is expected that monolayer culture represents optimal conditions in terms of diffusive transport, and therefore association and internalization would be lower in the 3D tumor environment.
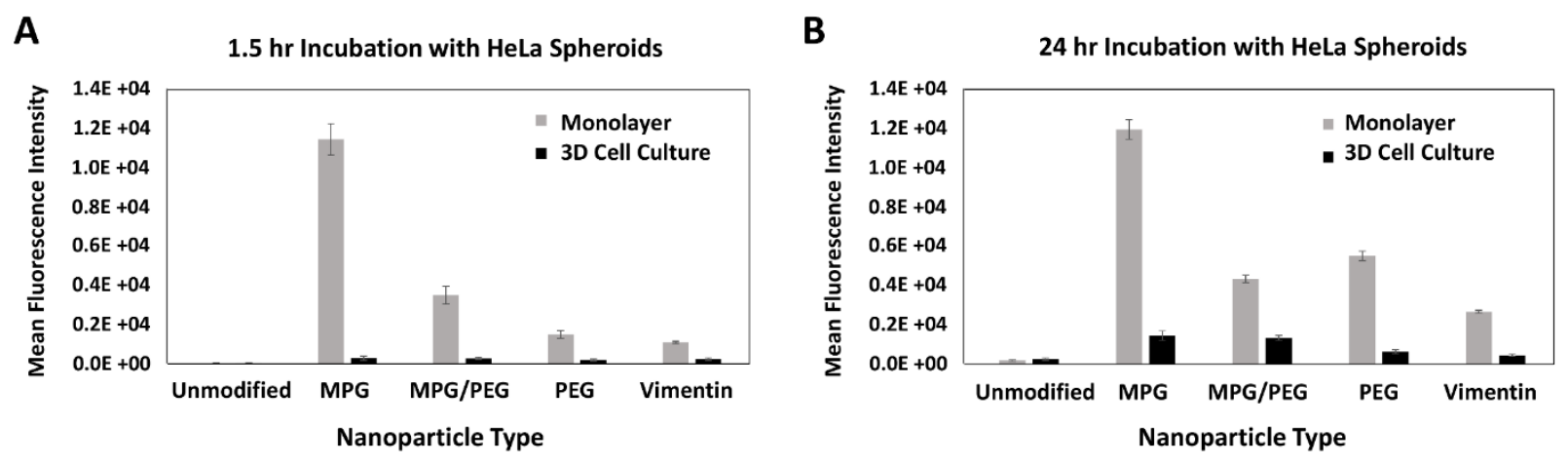

Figure 6. Comparison of NP cellular internalization between HeLa monolayer and spheroid cell cultures. Results are shown at (a) 1.5 and (b) $24 \mathrm{~h}$

Transport of PLGA-modified nanoparticles in hypo-vascularized cancer lesions

The NP diffusion profiles after $1.5 \mathrm{~h}$ through the spheroid tissue are presented in Fig. 7, showing that MPG and PEG-modified NPs exhibited the greatest fluorescence intensity averaged over $200 \times 80 \mu \mathrm{m}$ areas, followed by MPG/PEG and VIM NPs. In particular, MPG NPs were heavily detected in the periphery (within $100 \mu \mathrm{m}$ of the edge) of the spheroids, viz., $2 \times, 1.4 \times$, and $2.7 \times$ that of PEG, MPG/PEG, and VIM NPs, respectively, while PEG NPs penetrated $2 \times$ farther $(250$ $\mu m$ ) into the spheroid than the MPG NPs. In comparison, unmodified NPs exhibited the lowest detection and penetration into the tumor spheroids. 


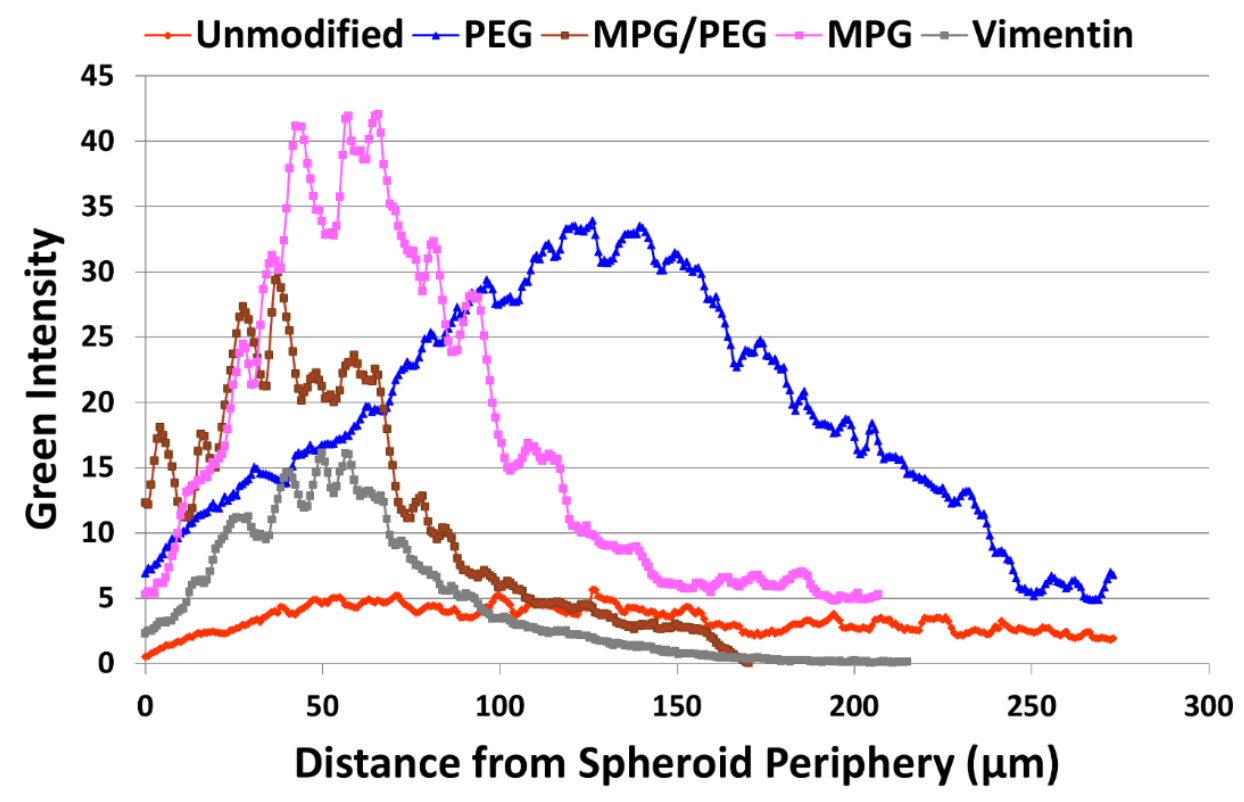

Figure 7. NP penetration into 3D cell culture (HeLa spheroids) after 1.5hr incubation.

\section{DISCUSSION}

Building upon our previous work using avidin-palmitate PLGA NPs with modified surface chemistry, here we study the effects of contrasting and hybrid surface modifications on NP transport and cellular internalization in cervical tumor cells. Previously, avidin-palmitate NPs conjugated with the cell-penetrating peptide MPG were shown to have rapid internalization in monolayer cervical carcinoma (HeLa) cells, with a significant improvement over unmodified NPs. Here, we expand the suite of NP surface modifications to include PEG, VIM, and a hybrid modification of MPG and PEG. These formulations were evaluated for their internalization over short and extended time periods (1.5 and $24 \mathrm{~h}$, respectively) in monolayer cell culture, representing optimal transport conditions, and in tumor spheroid cultures, representing hypovascularized tumor lesions with diffusive transport limitations. Additionally, we extend our 
analysis beyond cancerous cells to include normal vaginal cells (VK2) to assess potential differences in the efficacy of different surface modifications when targeting tumor versus nontumor cells present in the female reproductive tract.

The NP formulations were characterized using standard methods to evaluate size, hydrodynamic size, and zeta potential. Unmodified NPs exhibited the most negative surface charge, while surface modification increased the surface potential to more positive values. MPG NPs exhibited the greatest increase in surface potential, as MPG is an amphipathic peptide that contains a cationic sequence known to enhance cell surface interaction. All NP surface modifications yielded an improvement in cellular association and internalization over unmodified NPs under optimal transport conditions in HeLa monolayer cell culture (Fig. 3a, b). MPG-modified NPs had the highest cellular association and internalization at both 1.5 and $24 \mathrm{~h}$, indicating their potential to efficaciously promote NP uptake. The hybrid MPG/PEG NPs showed significantly enhanced uptake at $1.5 \mathrm{~h}$, greater than both PEG and VIM-modified NPs. However, PEG significantly increased cellular internalization after a longer exposure time and surpassed the hybrid MPG/PEG NPs. The MPG, VIM, and hybrid NPs showed modest improvement in internalization after longer treatment duration. These results are in agreement with our previous work that MPG-modified NPs have a rapid mechanism of uptake in HeLa cells while PEG-modified NPs take longer to internalize. This is consistent with PEG functionality, which is designed to prolong half-life in vivo.

In contrast, in normal vaginal cells (VK2), PEG-modified NPs exhibited similar high uptake and internalization at 1.5 and $24 \mathrm{~h}$ (Fig. 4c, d), while MPG and MPG/PEG NPs showed only slight internalization. In fact, the MPG-modified NPs demonstrated greater than threefold improvement in internalization by cancerous relative to normal cells. These results suggest that the association 
and uptake of these NPs in normal cells may be mediated by different molecular mechanisms or cell surface moieties than in cancerous cells, which may have significant implications in more specific targeting of cancer lesions. The results further suggest that while VIM NPs are more efficacious than unmodified NPs, VIM most likely does not serve a specific targeting purpose for these cells. Enhancement is most likely due to the slightly more positive surface potential compared to that of the unmodified NPs. The high internalization of PEG-coated NPs in the VK2 cells, and also after $24 \mathrm{~h}$ in HeLa cells, indicates that these NPs might provide a means to enhance transport and concentration of therapeutics into normal vaginal tissue, while also offering the potential for enhanced uptake in cancerous cells.

Following the assessment of cellular internalization in optimally diffusive (monolayer) conditions, a similar evaluation was performed in 3D cell culture of HeLa cells to assess the transport and uptake of these NPs in hypo-vascularized tumor tissue. After the short incubation of $1.5 \mathrm{~h}$, all surface-modified NPs performed similarly (Fig. 5), demonstrating slightly increased internalization relative to the unmodified NPs. After $24 \mathrm{~h}$, the MPG and MPG/PEG NPs exhibited the most internalization relative to the other NP groups. Unlike the monolayer cultures, none of the groups saturated the spheroids at $1.5 \mathrm{~h}$, and instead elicited a more gradual, yet significant increase in internalization with longer exposure time. These results highlight the diffusive transport limitation through the 3D tissue culture as a time-dependent process, and enable a more detailed assessment of NP performance. The transport limitation in 3D tissue is well known (e.g., ${ }^{140-144}$ ), and derives from the extra-cellular matrix as well as cell-matrix and cell-cell interactions (such as E-cadherin) impeding diffusive flow ${ }^{145,146}$. Whereas in monolayer HeLa and VK2 cell cultures the MPG and PEG NPs were internalized the most compared to the other groups, in the 3D cell culture the MPG and MPG/PEG NPs showed highest internalization. 
The diffusive transport limitation is further emphasized in Fig. 5, which compares the 2D and 3D internalization of the NPs in monolayer versus spheroid cultures.

As shown in Fig. 7, PEG NPs have the greatest depth of penetration into the spheroid tissue, with maximal concentration reached at $125 \mu \mathrm{m}$. In contrast, the MPG NPs achieved the highest concentration along the tumor periphery (maximally at $65 \mu \mathrm{m}$ ), but were unable to fully penetrate it. As shown in the monolayer, MPG NPs have the highest rate of cellular internalization, which is critical to the success of some biological therapeutics that cannot transverse cellular membranes alone, such as oligonucleotides, but this increased internalization may limit the depth of penetration. In contrast, the PEG modification, being very hydrophilic and less positively charged, enables NPs to more easily navigate the extracellular space and penetrate deeper into the spheroid tissue at the expense of internalization.

In living subjects, circulating NPs can preferentially lodge in tumor tissue by extravasating from fenestrated capillaries ${ }^{147}$ as a result of the enhanced permeability and retention effect (EPR). Nevertheless, it has been shown that NP diffusive transport only extends about 30-50 $\mu \mathrm{m}$ away from the vessels ${ }^{148,149}$. Consequently, therapeutics released from these NPs may not attain cytotoxic concentrations and also fail to affect quiescent (non-cycling) cells in regions distal to the vasculature ${ }^{150}$. Previously, we evaluated drug and NP transport in 2D and 3D cell culture, showing that nanoparticles layered with combinations of hexadecanethiol, phosphatidylcholine and high-density lipoprotein diffused deeper than PEGylated NPs into 3D cell culture representing hypo-vascularized tissue ${ }^{151}$. The NPs were uptaken in solid tumor tissue in vivo ${ }^{152}$, and elicited distinctive drug release kinetics when loaded with paclitaxel or cisplatin ${ }^{153}$. Cytotoxicity experiments with free drug showed a substantial differential between 2D and 3D cell cultures, highlighting the increased resistance due to diffusive transport ${ }^{124}$, although the 
drug-loaded layered NPs were substantially more efficacious in 3D cell culture than free-drug. While these findings are consistent with previous work showing decreased efficacy in 3D cell culture compared to monolayer ${ }^{122,148}$, they offer hope that modification of NPs to enhance their uptake and transport in cancerous tissue can help overcome the limitations of diffusive transport. 
III. Distribution of PLGA-modified nanoparticles in 3D cell culture models of hypo-vascularized tumor tissue

Adapted From:

Sims LB, Huss M, Frieboes H, Steinbach-Rankins JM; Distribution and Uptake of PLGA-modified Nanoparticles to Target Heterogeneously Vascularized Cervical Cancer Lesions; October 5, 2017; Journal of Nanobiotechnology ${ }^{1}$

\section{A. CLINICAL SIGNIFICANCE AND BACKGROUND}

Relative to effective and non-invasive preventative options such as vaccines, late-stage cancer treatments are usually invasive and painful, and typically include surgery, chemotherapy, and radiation treatment. Chemotherapy often induces irreversible damage to surrounding healthy tissue as well as incomplete tumor eradication. For systemic chemotherapy specifically, it can be challenging to achieve distribution throughout the tumor to maximize treatment effectiveness. Nanotherapeutic platforms have been proposed as safer and more effective modalities to deliver therapeutic agents directly to the tumor site. In particular, FDA-approved polymer-based platforms such as poly(lactic-co-glycolic) acid (PLGA) NPs, have been utilized to reduce unwanted immunogenic responses. Although NPs have been surface-modified with a variety of ligands to enhance tumor penetration and targeting $8,17,28,29,115,154-158$, currently, two delivery paradigms exist, often with cellular internalization and tissue penetration diametrically opposed. In trying to achieve enhanced cellular internalization, the efficacy benefit may be limited if surface-modification prevents the carrier from penetrating deeply into the tumor interstitium ${ }^{28}$. Conversely, if penetration into the tumor interstitium is successfully achieved - thereby providing broad distribution throughout the tumor - delivery vehicles may be inadequately internalized by the cells targeted. Unfortunately, similar ineffective therapy results in both cases. 
To balance these transport challenges, NPs are often surface-modified with ligands to enhance transport and longevity after localized or systemic administration. One of the most common ligands used to functionalize and promote NP delivery, poly(ethylene-glycol) (PEG), has been employed as a "stealth" modification, due to its hydrophilic and easily tailorable properties. PEG has been shown to increase vehicle circulation time by decreasing unwanted systemic interactions, and has enhanced transport through interstitial space and intercellular junctions 2,13,29,30,75,79,151,159-161. In contrast, cell penetrating peptides (CPPs) - short amphipathic or polycationic peptides - have been utilized to improve the intracellular delivery of cargo. Due to their cationic and sometimes lipophilic properties, CPPs have been designed to promote the internalization of attached cargo across cell membranes, particularly for gene delivery applications $28,115,154,155,158,160,162$.

For cervical cancer specific applications, a variety of polymeric NP formulations have been recently investigated to deliver chemotherapeutics. Nanoparticle derivatives of PLGA 157,163-165 have demonstrated sustained delivery of docetaxel against cervical cancer both in vitro and in vivo, correlated with high uptake and corresponding antitumor effects. Similarly, Eudragit-E and polyvinyl alcohol NPs containing Naringenin induced dose-dependent cytotoxicity ${ }^{166}$. In another study, genistein-encapsulated $\varepsilon$-caprolactone-based NPs demonstrated enhanced cytotoxicity and growth inhibition in a murine HeLa xenograft tumor model ${ }^{167}$. Folate-targeted doxorubicinloaded NPs have improved targeting and anti-tumor efficacy in vivo ${ }^{161}$ and pullulan acetate folate-modified NPs were used to treat cervical carcinoma ${ }^{167}$. Peng et al. utilized a thermosensitive gel to target DNA poly( $\beta$-amino ester) NPs to ex vivo pre-neoplastic cervical lesions and mouse cervical tissue ${ }^{168}$. Similarly, Blum et al. developed topical camptothecinloaded PLGA nanoparticles to prevent tumor growth in an inducible murine model of vaginal 
squamous cell carcinoma ${ }^{169}$. Most recently, Yang et al. evaluated paclitaxel mucus penetrating or adhesive PLGA NPs, demonstrating significantly less tumor growth and increased survival with mucoadhesive NPs ${ }^{35}$.

Previously, we investigated the effects of NP surface-modification with cell-penetrating peptides (CPPs), stealth ligands, and tumor targeting ligands (MPG (unabbreviated notation), PEG, and Vimentin, respectively), on NP penetration and distribution within the hypo-vascularized tumor environment ${ }^{2}$. Nanoparticles modified with a CPP, MPG, exhibited the highest cellular internalization in human cervical carcinoma (HeLa) 3D cell culture (multi-cellular spheroids); however, internalization primarily occurred within the spheroid periphery, resulting in a modest $(<100 \mathrm{~nm})$ distribution profile. In contrast, PEG-modified NPs distributed more deeply into spheroids, but were less readily internalized by cells. These results seemed to indicate that tissue morphology in addition to NP functionalization were key factors determining NP distribution.

In this study, we consider tissue morphology while evaluating the penetration and distribution of PEG, cell penetrating (MPG), and CPP-stealth (MPG/PEG) NP co-treatment strategies in models of hypo-vascularized cervical cancer tissue representing regions distal from the point of vascular extravasation (Fig. 1). To model more regularly-shaped tissue, we utilized the hanging drop (HD) method to generate smaller, more homogeneously spherically-shaped spheroids. We compared NP penetration to that in tissue formed via the liquid overlay (LO) technique, in which spheroids form more irregularly-shaped masses. To compare differences in NP distribution within the same type of lesion but as a function of different cervical cancer types, we selected HeLa, cervical epithelial adenocarcinoma cells; CaSki, cervical epidermoid carcinoma cells; and SiHa, grade II cervical squamous cell carcinoma cells. Thus, the goal was to assess differences in NP distribution as a function of NP surface-modification (unmodified, MPG, PEG, or MPG/PEG 
NP co-treatment), hypo-vascularized tissue (regular or irregular morphology), and cancer cell type.

\section{B. METHODS}

\section{Synthesis of avidin-palmitate conjugates}

Avidin-palmitate was synthesized for subsequent conjugation to NPs as previously described ${ }^{2,136}$. Briefly, $40 \mathrm{mg}$ of avidin (A9275, Sigma) was dissolved in $4.8 \mathrm{~mL}$ of $2 \%$ sodium deoxycholate (NaDC) in phosphate buffered saline (PBS) warmed to $37^{\circ} \mathrm{C}$. Palmitic acid-NHS (PA-NHS, Sigma) was dissolved in $2 \% \mathrm{NaDC}$ to a final concentration of $1 \mathrm{mg} / \mathrm{mL}$ and sonicated until wellmixed. PA-NHS solution $(3.2 \mathrm{~mL})$ was added dropwise to the avidin NaDC solution, and reacted overnight at $37{ }^{\circ} \mathrm{C}$. The following day, the reaction was dialyzed in $1200 \mathrm{~mL}$ of $0.15 \% \mathrm{NaDC}$ in PBS heated to $37^{\circ} \mathrm{C}$. Free PA-NHS was dialyzed overnight at $37^{\circ} \mathrm{C}$ using $3500 \mathrm{MWCO}$ tubing to remove free palmitic acid. After overnight dialysis, the dialysis tubing contents were transferred to a storage vial and stored at $4{ }^{\circ} \mathrm{C}$ until use.

\section{Nanoparticle synthesis}

PLGA NPs encapsulating the fluorophore Coumarin 6 (C6) were synthesized as previously described $^{2,39}$ to enable visualization via fluorescence microscopy. From earlier studies ${ }^{33,137-139}$, as well as our previous experiments ${ }^{2,28}$, we have observed that negligible quantities $(\sim 1 \%)$ of C6 are released from NPs. This is attributed to the hydrophobic nature of C6 encapsulated within hydrophobic NPs. Therefore, C6 detected in cells reflects NP distribution in or bound to the cells, not C6 release and distribution. Briefly, C6 NPs were synthesized using an oil-in-water (o/w) single emulsion technique ${ }^{2,13,39}$. Carboxyl-terminated poly(lactic co-glycolic acid, PLGA) $\left(0.55-0.75 \mathrm{dL} / \mathrm{g}\right.$, LACTEL $\left.^{\circledR}\right)$ was used to synthesize $100-200 \mathrm{mg}$ batches. Coumarin 6 was 
dissolved in methylene chloride (DCM) overnight at a concentration of $15 \mu \mathrm{g}$ C6 per mg of PLGA. The following day, the PLGA/C6/DCM solution was added dropwise to a 5\% polyvinyl alcohol (PVA) solution of equal volume, vortexed and sonicated. The resulting NPs were hardened in $0.3 \%$ PVA during solvent evaporation for $3 \mathrm{hr}$.

Unmodified NPs were washed after hardening, and centrifuged at $4{ }^{\circ} \mathrm{C}, 3$ times in deionized water (diH2O) to remove residual solvent. NPs were frozen, lyophilized, and stored at $-20{ }^{\circ} \mathrm{C}$ until use. A similar protocol was followed to synthesize MPG (3177 Da, GenScript) and PEG (5000 Da, Nanocs Inc.) modified NPs, with the addition of avidin-palmitate (1 mg/mL) to the 5\% PVA solution. Surface-modified NPs were collected after the first wash, and incubated for 30 min with biotinylated ligands at a molar ratio of 3:1 ligand:avidin in PBS. After the conjugation reaction, the NPs were washed two more times with diH2O centrifugation, frozen, and lyophilized. All NPs were stored at $-20^{\circ} \mathrm{C}$ after synthesis.

\section{Nanoparticle characterization}

NP characterization confirmed physical properties including NP diameter, morphology, and surface charge. First, scanning electron microscopy (SEM, Zeiss SUPRA 35VP) was utilized to verify NP morphology. Unhydrated NP diameters were measured using NIH ImageJ software. NP surface charges were characterized using a Malvern Zetasizer (Zetasizer Nano ZS90).

\section{Three-dimensional cell cultures}

The human cervical carcinoma cell lines, SiHa, and CaSki (ATCC), were kindly provided by Dr. Alfred Jenson (University of Louisville), while the HeLa cell line was generously provided by

Dr. Kenneth Palmer (University of Louisville). HeLa and SiHa cells were maintained in Minimum Essential Media (MEM) and CaSki cells were maintained in RPMI medium, both 
supplemented with $10 \%$ fetal bovine serum and $1 \%$ penicillin-streptomycin. All cells were kept in a humidified atmosphere of $5 \% \mathrm{CO} 2$ at $37{ }^{\circ} \mathrm{C}$, and were grown to $80 \%$ confluence prior to tumor spheroid formation.

\section{Liquid overlay tumor spheroid formation}

Liquid overlay spheroids were grown as previously described ${ }^{2,50}$.. Briefly, to prevent spheroid adherence to the plate, 24-well tissue culture plates (\#353047, Corning) were coated with a $1 \%$ (w/v) agarose gel $6 \mathrm{~h}$ prior to spheroid formation. After trypsinization, cells were collected and plated at a density of 100,000 cells per well containing $700 \mu \mathrm{L}$ culture medium and lightly shaken $(100 \mathrm{rpm})$ for $15 \mathrm{~min}$ on a reciprocating shaker. Following this, culture plates were transferred to an incubator and maintained at $37{ }^{\circ} \mathrm{C}$ and $5 \% \mathrm{CO}$. Spheroids were grown for 7 days with culture media changes every 2 days (Fig. 1).

\section{Hanging drop tumor spheroid formation}

To form hanging drop spheroids, ultra-low attachment plates (\#4515, Corning) were utilized. Briefly, cells were trypsinized after reaching 80-90\% confluency and seeded at a density of 5000 cells per well in $100 \mu \mathrm{L}$ culture medium. Care was taken to minimize pipette tip contact with ultra-low attachment plate well walls. Spheroids were allowed to form for 5 days under the cell culture conditions described above (Fig. 1). 


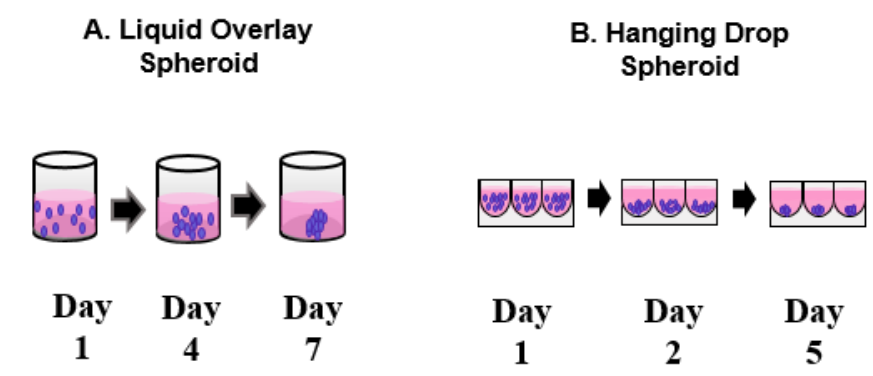

Figure 1. Schematic of spheroid formation techniques for a liquid overlay (LO) and b hanging drop (HD) spheroids, respectively representing larger, more irregularly-shaped and smaller,

regularly-shaped avascular tissue

Spheroid characterization

Spheroid morphology was characterized prior to NP administration. Briefly, spheroids were removed from culture plates and placed on imaging dishes (P35G-0-14-C, MatTek) in $25 \mu \mathrm{L}$ of culture medium to prevent drying. Spheroids were then imaged using an epifluorescent microscope (Axiovision 4, Zeiss) under transmitted light using a $10 \times$ objective (Fig. 2).

\section{NP distribution}

To assess NP distribution in tumor spheroids, four different NP formulations were evaluated: unmodified, PEG, MPG, and MPG/PEG co-treatment groups. Once spheroid formation was achieved using the hanging drop (5 days) and liquid overlay (7 days) techniques, fresh culture medium was added and spheroids were incubated with $10 \mu \mathrm{g} / \mathrm{mL}$ NPs for $1.5 \mathrm{~h}$. For the NP cotreatment group, a half-dose of the MPG $(5 \mu \mathrm{g} / \mathrm{mL})$ and PEG $(5 \mu \mathrm{g} / \mathrm{mL})$ NPs were combined for a total NP dose of $10 \mu \mathrm{g} / \mathrm{mL}$. After NP administration, both LO and HD individual tumor spheroids, were transferred to eppendorf tubes for fluorescent staining. Due to the differing spheroid sizes that result from the LO and HD methods, the total volume transferred was either 
100 or $50 \mu \mathrm{L}$, for LO and HD spheroids, respectively. Once transferred, tumor spheroids were washed with $0.2 \mathrm{~mL}$ of $1 \mathrm{X}$ PBS and fixed with $0.2 \mathrm{~mL}$ of $4 \%$ paraformaldehyde for $10 \mathrm{~min}$ at RT. Subsequent to fixation, spheroids were treated with $0.2 \mathrm{~mL}$ of $1 \%$ Triton-X for $10 \mathrm{~min}$ at RT. Spheroids were subsequently washed twice with $0.2 \mathrm{~mL}$ of PBS, followed by treatment with $0.2 \mathrm{~mL}$ of $4 \mu \mathrm{g} / \mathrm{mL}$ Hoescht in 1\% BSA PBS++ (containing $\mathrm{CaCl} 2$ and $\mathrm{MgCl} 2$ ) for $10 \mathrm{~min}$ at $\mathrm{RT}$ for nuclear staining. Finally, spheroids were washed in $0.2 \mathrm{~mL}$ of PBS and once in $0.2 \mathrm{~mL}$ of DI water. Spheroids were then transferred to imaging dishes (P35G-0-14-C, MatTek) suspended in $50 \mu \mathrm{L}$ PBS.

NP uptake and distribution through tumor spheroids were assessed via confocal microscopy (LSM 710, Zeiss) and image analysis was performed using ImageJ software. The following laser settings: 4' 6-diamidino (DAPI) and GFP were used to visualize Hoechst (blue, cell nuclei) and C6 (green, within NPs), respectively. A laser intensity of 2 and a gain of 600 were used for the DAPI/Hoechst channel, while a laser intensity of 5 and a gain of 500 were maintained for the GFP/C6 channel across experiments. Imaris x64 (v7.7.2, Bitplane) was utilized to generate 3D images from the composite $\mathrm{z}$-stacks of the tumor spheroids. These 3D images were then rotated $90^{\circ}$, forming cross-sections of the tumor spheroids (figure not shown). Using ImageJ, at least 3 representative samples were taken from the tumor cross-sections to evaluate NP distribution within the spheroids. NP penetration was quantified by plotting the mean fluorescence intensity (MFI) of each 3D optical reconstruction as a function of distance from the periphery of the spheroid towards the midplane. At least 8 fields of view were analyzed per sample, of which the averages and standard deviations are reported. NP internalization was then assessed by analyzing the area under the curve (AUC, MFI- $\mu \mathrm{m})$ of the generated distribution profiles using a 
trapezoidal approximation in Excel. Statistical significance of NP treatment group penetration was determined using a one-way ANOVA post hoc Tukey test $<0.05$.

\section{RESULTS}

To assess the distribution of unmodified, PEG, MPG, or MPG/PEG co-treatment NPs into hypovascularized cervical cancer tissue regions distal from the point of vascular extravasation, NP diffusion was measured in SiHa, CaSki, or HeLa LO or HD spheroids. The area under the curve (AUC, MFI- $\mu \mathrm{m}$ ) was determined, corresponding to the fluorescence intensity of NPs distributed throughout the tumor spheroid, in addition to the maximum mean fluorescence intensity (MFI) observed at a given penetration distance for each spheroid and cell type. The maximum MFI defines the spheroid depth at which the highest MFI was observed within the tumor spheroid after $1.5 \mathrm{~h}$ administration. NP penetration and distribution through the spheroids varied as a function of surface modification, tissue morphology (LO or HD), and cell type (Figs. 3, 4, 7).

\section{NP characterization}

NP size and morphology were confirmed using SEM imaging and ImageJ processing. Unhydrated NPs demonstrated a spherical morphology, with diameters measuring 160 to180 $\mathrm{nm}$ 2. Hydrated NP surface charges were measured using a Zetasizer (Malvern). Unmodified NPs had a negative surface charge of $-26.63 \pm 1.05 \mathrm{mV}$; while PEG- and MPG-modified NPs measured $-22.03 \pm 1.40$ and $-8.54 \pm 0.35 \mathrm{mV}$, respectively (Table 1), validating surface ligand conjugation. 
Table 1. Zeta potentials of unmodified, PEG-, and MPG-modified NPs.

\begin{tabular}{|c|c|}
\hline & NP Zeta-Potential (mV) \\
\hline Unmodified & $-26.63 \pm 1.05$ \\
\hline PEG & $-22.03 \pm 1.40$ \\
\hline MPG & $-8.54 \pm 0.35$ \\
\hline
\end{tabular}

\section{Spheroid characterization}

In general, the HD spheroids were smaller in size for HeLa, CaSki, and SiHa cells (with respective average maximum cross-sectional areas of $0.31,0.10$, and $0.25 \mathrm{~mm} 2$ ) compared to the LO spheroids (with respective areas of $0.55,0.39$, and $0.57 \mathrm{~mm} 2$ ) - see Table 1 . In addition, for both HD and LO spheroids, CaSki tumors demonstrated statistically significant decreases in size, relative to HeLa or SiHa spheroids.
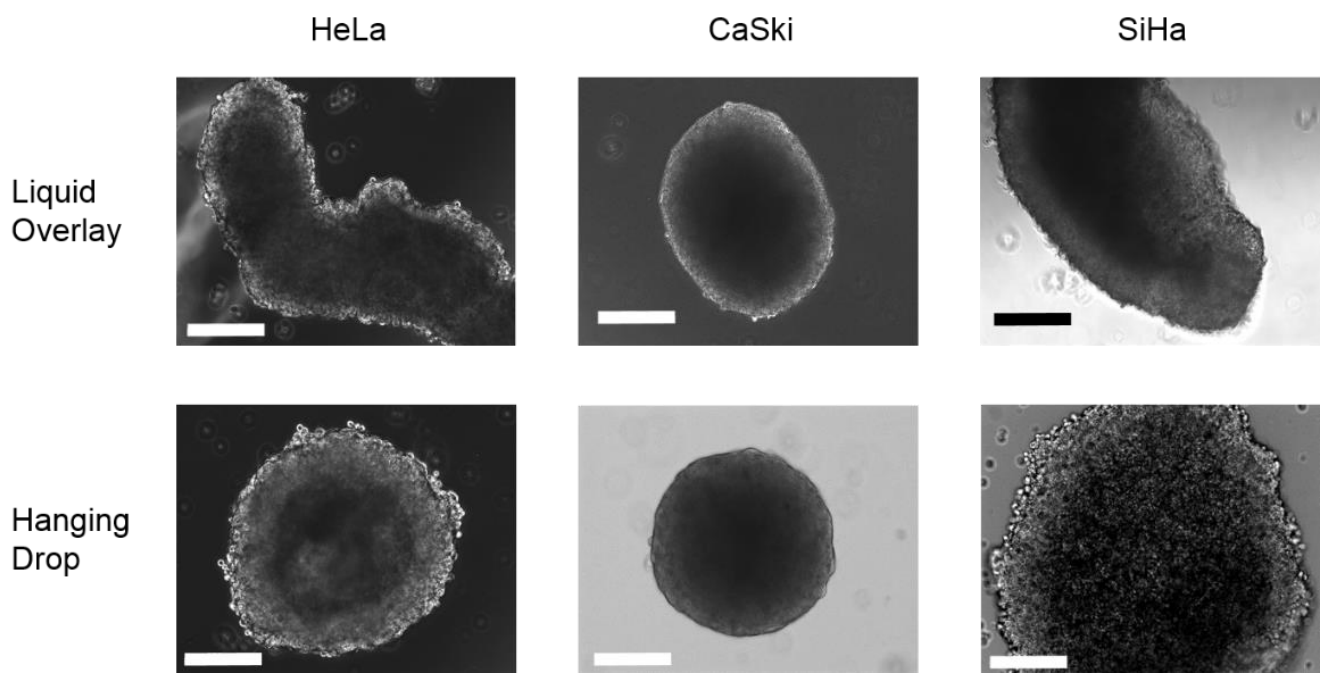

Figure 2. Typical morphologies of liquid overlay (LO) and hanging drop (HD) tumor spheroids, evaluated via bright feld microscopy. Scale bar: $200 \mu \mathrm{m}$ 
Table 2. Maximum cross-sectional areas of hanging drop (HD) and liquid overlay (LO) tumor spheroids as a function of cell type. All values represent the average \pm standard deviation $(n=3)$.

\begin{tabular}{|l|c|c|}
\hline \multicolumn{3}{|c|}{ Tumor Spheroid Surface Area $\left(\mathrm{mm}^{\wedge}\right.$ 2) } \\
\hline & Hanging Drop & Liquid Overlay \\
\hline HeLa & $0.31 \pm 0.02$ & $0.55 \pm .03$ \\
\hline CaSki & $0.10 \pm 0.02$ & $0.39 \pm 0.04$ \\
\hline SiHa & $0.25 \pm 0.03$ & $0.57 \pm 0.04$ \\
\hline
\end{tabular}

NP distribution as a function of cell type and NP formulation was quantified for LO (Fig. 5) and HD (Fig. 6) spheroids, respectively. The AUC as a function of NP treatment group and cell type are plotted in Fig. 7. Table 3 provides the actual AUC values for each treatment and cell type. Despite the minimal distribution observed with unmodified NPs, a statistically significant difference was observed between regularly-shaped (HD) and irregularly-shaped (LO) HeLa spheroids (AUCs LO $6827 \pm 3101$ and HD 15,841 \pm 1637 MFI- $\mu \mathrm{m}$ ), while no statistical differences were observed between regularly- and irregularly-shaped CaSki or SiHa cell spheroids. Furthermore, unmodified NPs exhibited similar distribution trends within HeLa, CaSki, and SiHa spheroids of each type (Figs. 3-6). Within all spheroids, the distribution profiles showed that the maximum MFI occurred within $100 \mu \mathrm{m}$ of the tumor periphery. Moreover, only SiHa HD spheroids exhibit a maximum MFI peak above 100 MFI (Fig. 4 and Table 4), indicating substantial accumulation of NPs at a given penetration distance. Overall, unmodified NPs show no differential penetration and distribution between tumor/cell types and exhibited only modest distribution within spheroids, relative to surface-modified NP groups. 


\section{MPG NP penetration and distribution}

In contrast to the similar distribution profiles of unmodified NPs within a given spheroid or cell type, significant differences in distribution were observed for MPG NPs as a function of tissue morphology and cell type. In HeLa spheroids, MPG NP distribution (AUC) was found to be statistically significant and three times higher in regularly-shaped (HD) spheroids (47,018 \pm $8751 \mathrm{MFI}-\mu \mathrm{m})$, compared to irregularly-shaped (LO) spheroids $(14,339 \pm 5129 \mathrm{MFI}-\mu \mathrm{m})$. Similarly, MPG NP distribution was doubled in SiHa regularly-shaped spheroids relative to SiHa irregularly-shaped spheroids (AUCs 24,972 \pm 2020 and 10,186 \pm 2887 MFI- $\mu$ ). However, a minimal difference in MPG NP distribution was observed between CaSki spheroids (Figs. 3-6 Table 3). Overall, MPG NPs distributed significantly more in HeLa spheroids (47,018 and 14,339 MFI- $\mu \mathrm{m})$, relative to the other cell types.

Additionally, MPG NPs provided much greater intratumoral accumulation when compared to unmodified NPs, and the distribution trends varied as a function of cell type. In regularly-shaped HD spheroids, MPG NPs penetrated deeper, and accumulated in greater amounts (represented by increased MFI) relative to irregularly-shaped LO spheroids. In HeLa regularly-shaped spheroids, NPs exhibited the greatest peak in accumulation $129 \mu \mathrm{m}(225 \mathrm{MFI})$ from the tumor periphery, with the second highest peak observed in SiHa cells (222 MFI) $91 \mu \mathrm{m}$ from the periphery (Fig. 4 and Table 4). Similar to irregularly-shaped spheroids, NP distribution through regularly-shaped CaSki spheroids was dampened by comparison.

In irregularly-shaped LO HeLa spheroids, MPG NPs demonstrated peaks $76 \mu \mathrm{m}$ (123 MFI) and $133 \mu \mathrm{m}$ (63 MFI) from the periphery (Fig. 3, Table 4). These values were significantly higher than exhibited by MPG NPs in irregularly-shaped LO SiHa (MFI 66) or CaSki (MFI 89) tumors. 
In contrast to these trends, CaSki cells demonstrated gentler and more uniform distribution curves, with less pronounced maximum MFIs, similar to unmodified NPs.

Overall, MPG NPs exhibited increased distribution in the smaller regularly-shaped spheroids relative to larger, irregularly-shaped spheroids, across all cell lines. HeLa and SiHa regularlyshaped spheroids seemed to offer the most permissive environments to MPG NP intratumoral distribution and accumulation.

\section{PEG NP penetration and distribution}

We observed that NPs modified with PEG exhibited variations in tumor penetration and distribution as a function of both cell and tissue type. In the larger, irregularly-shaped spheroids, PEG NPs had the greatest intratumoral distribution in SiHa cells (AUC 18,972 \pm 1065 MFI- $\mu$ ) and exhibited a bimodal distribution trend, relative to the dampened distribution observed in irregularly-shaped HeLa and CaSki spheroids (AUCs $4042 \pm 2101$ and $6718 \pm 1065$ MFI- $\mu$ m) (Figs. 3-6, Table 3). In contrast, significantly enhanced distribution of PEG NPs was observed within the regularly-shaped HD spheroids, with statistically significant increases in PEG NP distribution observed in HeLa and SiHa, relative to CaSki spheroids (AUCs 23,140 \pm 5531; $24,237 \pm 2532 ; 14,297 \pm 281$, respectively). Most notably, PEG NP distribution in regularlyshaped HeLa spheroids was nearly six-fold greater than in the irregularly-shaped tissue (Figs. 36, Table 3).

In regularly-shaped HD spheroids, PEG NPs exhibited similar unimodal distribution trends across all cell lines. SiHa regularly-shaped spheroids showed the greatest NP accumulation (238 MFI) occurring $78 \mu \mathrm{m}$ from the spheroid periphery (Fig. 4, Table 4). Moreover, NPs penetrated more deeply, and with higher accumulation, into regularly-shaped HeLa and SiHa spheroids (82 
and $78 \mu \mathrm{m})$, relative to regularly-shaped CaSki spheroids (64 $\mu \mathrm{m})$. In contrast, PEG NP distribution through the irregularly-shaped spheroids varied significantly as a function of cell type (Fig. 3, Table 4). In irregularly-shaped LO CaSki spheroids, the observed distribution profile was quite dampened, exhibiting a peak of maximum accumulation (62 MFI) only $39 \mu \mathrm{m}$ from the tumor periphery, followed by a decrease in penetration. In comparison, irregularlyshaped SiHa spheroids exhibited a bimodal distribution of PEG NPs, with minor and major maximum peaks (114 and $151 \mathrm{MFI}$ ) occurring 62 and $107 \mu \mathrm{m}$ from the spheroid periphery. In contrast, a maximum accumulation of only 42 MFI was reached $203 \mu \mathrm{m}$ from the spheroid periphery for HeLa cells (Fig. 3, Table 4). Overall, PEG NPs exhibited significant penetration and distribution in SiHa regularly- as well as irregularly-shaped spheroids, with a maximum intratumoral distribution observed in the regularly-shaped HeLa case.

\section{MPG/PEG co-treatment penetration and distribution}

The MPG/PEG NP co-treatment strategy also demonstrated differences in NP distribution as a function of tumor and cell type, similar to other modified NP groups. In regularly-shaped spheroids, the co-treatment groups demonstrated the greatest distribution in SiHa cells (AUC 26,970 \pm 5574 MFI- $\mu \mathrm{m}$ ) (Figs. 4, 6, and Table 3). Similarly, the MPG/PEG co-treatment NPs distributed well in HeLa regularly-shaped spheroids (AUC 15,657 \pm 3579 MFI- $\mu \mathrm{m}$ ), although there was no statistical significance observed in the AUC relative to regularly-shaped CaSki cells (Figs. 4, 6, and Table 3). In both CaSki spheroid types, the NP co-treatment group exhibited statistically decreased levels of distribution relative to that observed in HeLa and $\mathrm{SiHa}$ cells. In addition, for irregularly-shaped spheroids, insignificant differences in NP distribution were observed between HeLa and SiHa cells (AUCs 17,536 \pm 2675 and 19,783 \pm 1685 MFI- $\mu \mathrm{m}$ ), 
although the distribution trend differed between cell lines (Figs. 3, 5, Table 3). Overall, the MPG/PEG co-treatment had the strongest distribution trends (> 15,000 MFI- $\mu \mathrm{m})$ in the HeLa and SiHa spheroids, regardless of tumor type.

In terms of accumulation and penetration, within the regularly-shaped spheroids (Fig. 4, Table 4), the MPG/PEG NP co-treatment group exhibited the highest accumulation in SiHa cells (maximum peak $239 \mathrm{MFI}$ ), relative to that observed in HeLa (154 MFI) and CaSki cells (84 MFI). However, MPG/PEG NPs exhibited increased penetration in HeLa and CaSki regularlyshaped spheroids (102 $\mu \mathrm{m}$ and $90 \mu \mathrm{m}$, respectively). Additionally, SiHa and HeLa spheroids exhibited sharp unimodal peaks, whereas a more diminished distribution was observed in CaSki spheroids (Fig. 4). In HeLa and CaSki irregularly-shaped spheroids (Fig. 3), the trends were similar as observed in regularly-shaped spheroids, although NPs accumulated and penetrated less deeply in irregularly-shaped spheroids and exhibited more modest distribution. Overall, the MPG/PEG co-treatment NPs demonstrated the greatest distribution and penetration in SiHa and HeLa spheroids representing regularly- and irregularly-shaped avascular tissue. 


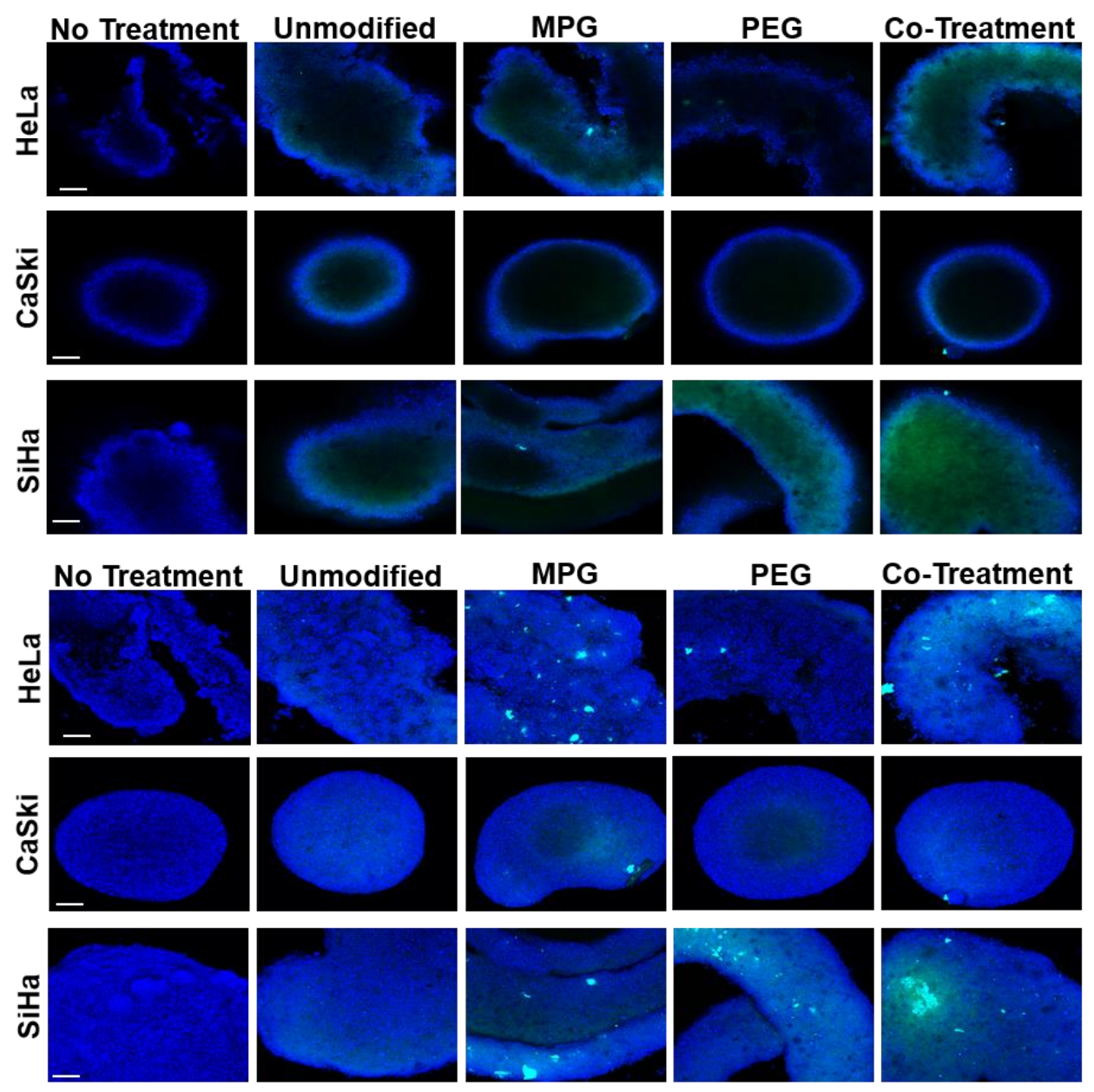

Figure 3. NP distribution through liquid overlay (LO) spheroids in mid-plane cross-sections (top three rows) and 3D composite (bottom three rows) confocal images. Nuclei are blue (Hoechst) and NPs are green (Coumarin 6). Scale bar: $50 \mu \mathrm{m}$ 


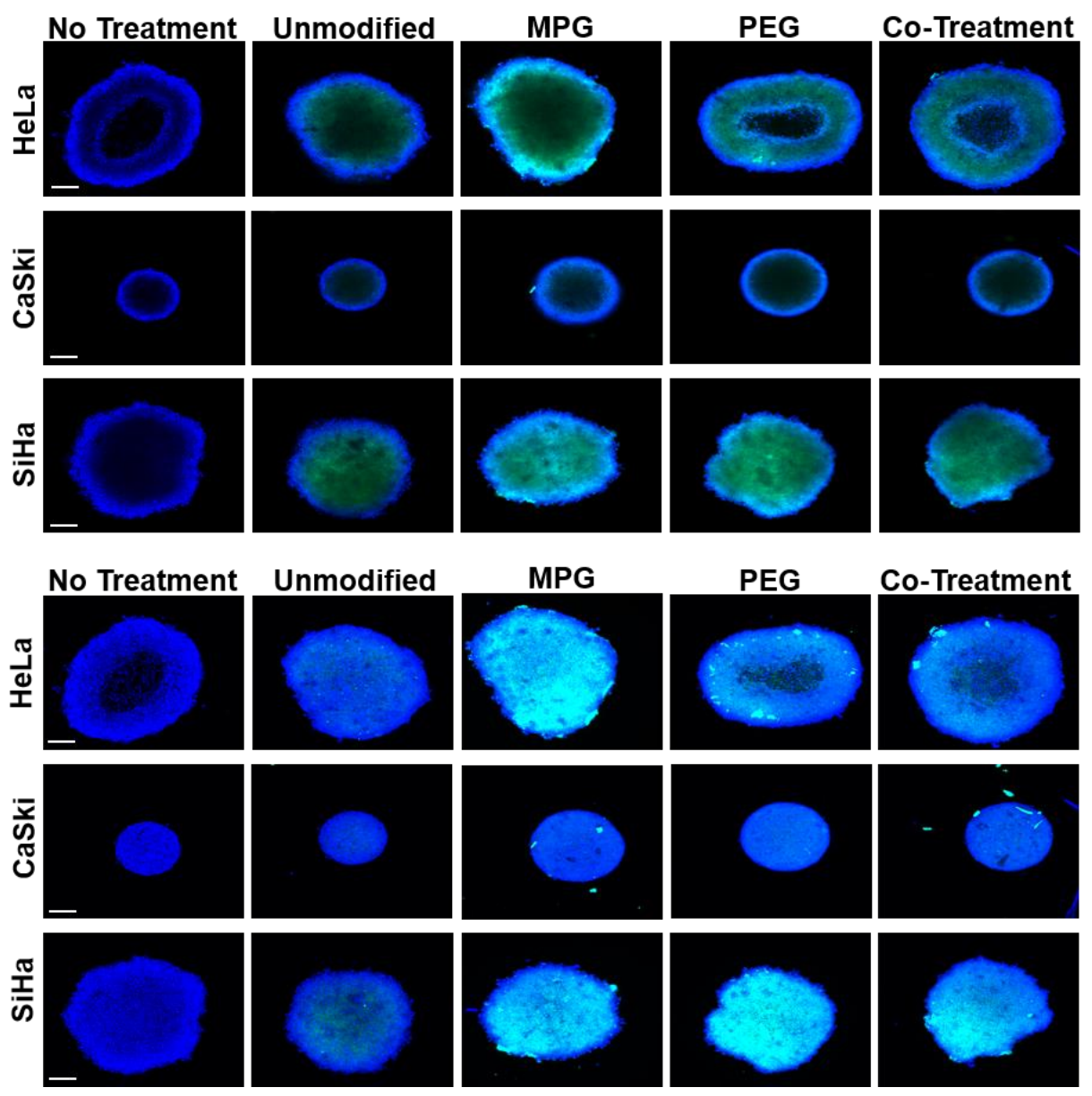

Figure 4. NP distribution through hanging drop (HD) spheroids in mid-plane cross-sections (top three rows) and 3D composite (bottom three rows) confocal images. Nuclei are blue (Hoechst) and NPs are green (Coumarin 6). Scale bar: $50 \mu \mathrm{m}$ 

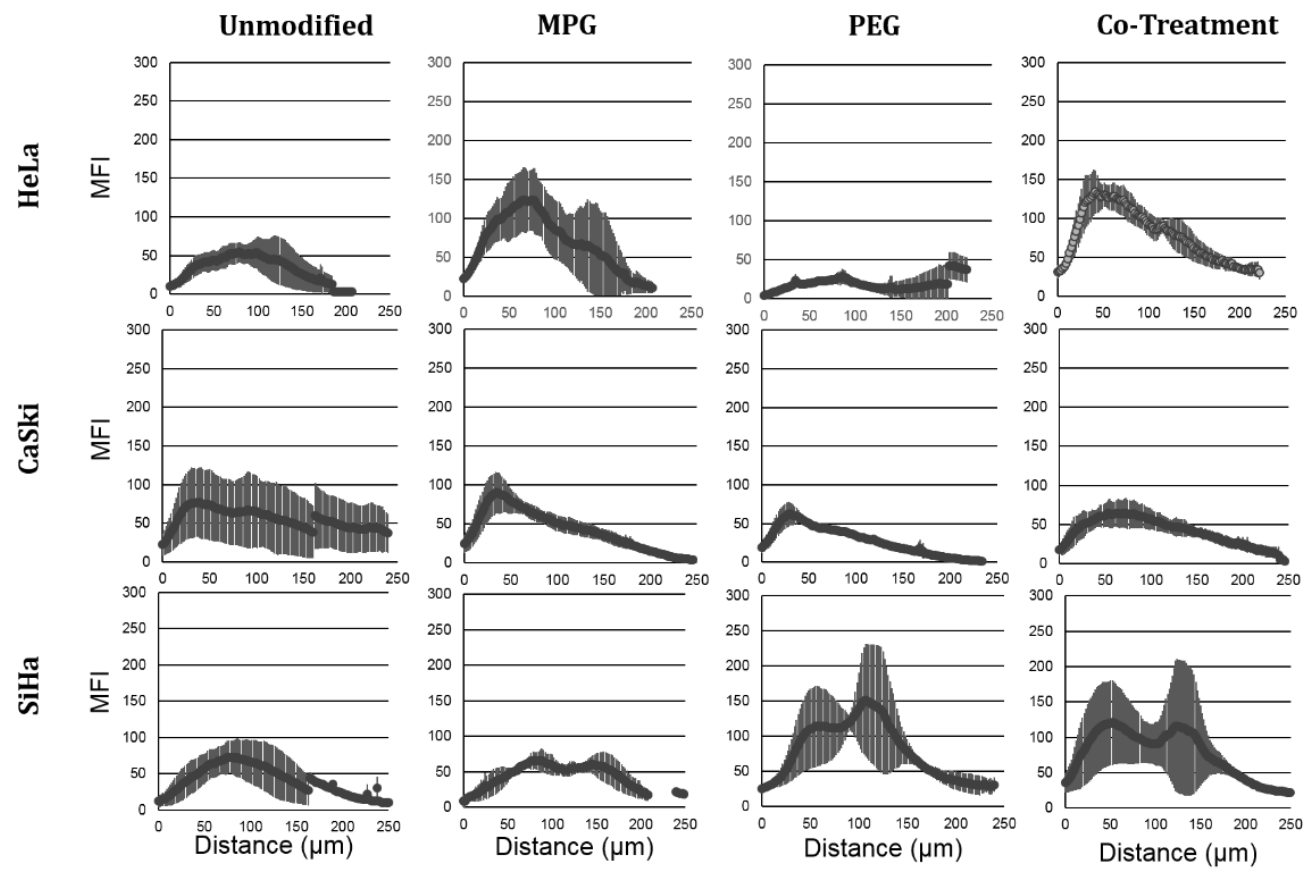

Figure 5. NP distribution profles quantifying the mean fuorescence intensity (MFI) vs.

penetration distance through liquid overlay (LO) spheroids. Distribution profles are shown as a function of NP treatment and tumor cell type. Average of the values along distance is denoted by the dark lines. 

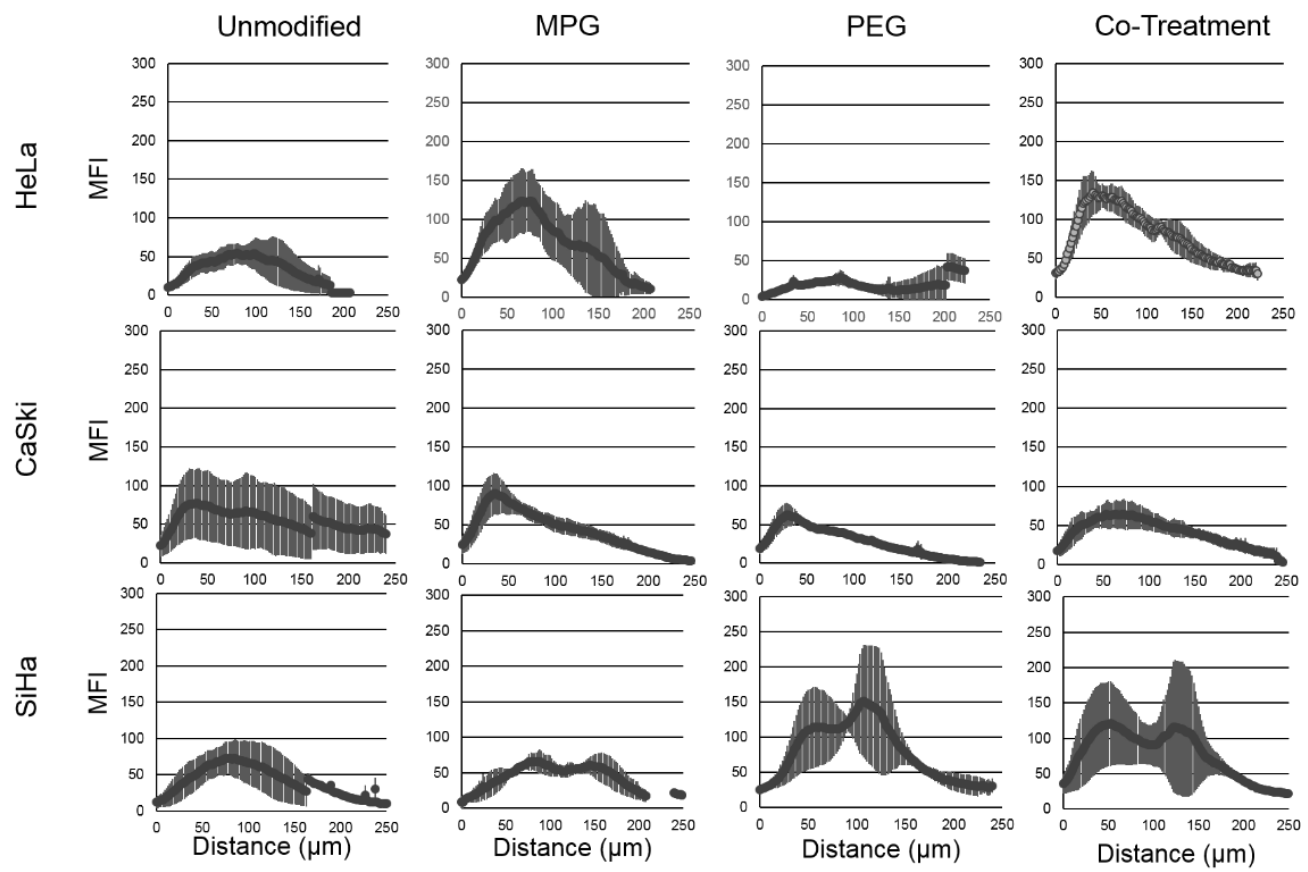

Figure 6. NP distribution profles quantifying the mean fuorescence intensity (MFI) vs.

penetration distance through hanging drop (HD) spheroids. Distribution profles are shown as a function of NP treatment and tumor cell type. Average of the values along distance is denoted by the dark lines. 


\section{DISCUSSION}

We have previously evaluated the effect of MPG, PEG, tumor-targeting (VIM), and hybrid (MPG + PEG) surface-modified PLGA NPs on NP internalization and distribution in HeLa cell monolayers and 3D LO spheroids ${ }^{2}$. We found that MPG-modified NPs offered the greatest internalization in monolayers and spheroids, and exhibited the highest intratumoral accumulation. Yet, despite high levels of distribution and internalization, the most effective particle group, MPG NPs, was sequestered within the tumor periphery, while PEG-modified NPs penetrated more deeply into the tumor interstitium. Additionally we noted that surface modification with opposing ligands on the same NP hindered penetration, which we attributed to potential steric hindrance or competing functionalities interacting with the ECM or tumor matrix. To expand upon this work, here we evaluate the impact of tumor size, tissue morphology, and cell origin on NP distribution. To assess these conditions, we used two different spheroid formation methods (LO and HD) to produce 3D spheroids that have been previously shown ${ }^{170}$ to respectively represent irregularly- or more regularly-shaped hypo-vascularized tumor regions distal from the location of vascular extravasation. We evaluated three different cervical carcinoma cell lines including HeLa, SiHa, and CaSki cells in both tumor models. Using the information gained from these studies, our goal was to provide insight into NP treatment strategies that may be applied to different types of tumors.

We observed that NPs achieved greater distribution in the smaller regularly-shaped spheroids, accompanied by increased penetration in HeLa and SiHa HD spheroids, relative to the larger, irregularly-shaped LO spheroids. Yet regardless of spheroid formation method, surface-modified NPs consistently exhibited increased penetration and distribution, relative to unmodified NPs. In irregularly-shaped spheroids, PEG and MPG/PEG co-treatment groups demonstrated the greatest 
distribution in SiHa cells (AUCs 18,972 \pm 1065 and 19,783 \pm 1685 MFI- $\mu \mathrm{m}$ ), followed by MPG and MPG/PEG in HeLa (AUC 14,339 \pm 5129 and 17,536 \pm 2675 MFI- $\mu \mathrm{m}$ ), whereas all NPs demonstrated rather modest penetration in irregularly-shaped CaSki spheroids. In comparison, in regularly-shaped spheroids, MPG exhibited the greatest distribution $(47,018 \pm 8754)$ in HeLa cells, with similar modest distribution of all NP groups through CaSki spheroids. In regularlyshaped SiHa spheroids, MPG, PEG, and MPG/PEG groups exhibited similar distribution (24,972 $\pm 2020,24,237 \pm 2532$, and $26,970 \pm 5574 \mathrm{MFI}-\mu \mathrm{m})$. In both regularly- and irregularly-shaped $\mathrm{SiHa}$ and HeLa spheroids, we observed only modest distribution of unmodified NPs, relative to other NP groups. In CaSki regularly-shaped spheroids, unmodified NPs demonstrated similar distribution $(12,043 \pm 5846 \mathrm{MFI}-\mu \mathrm{m})$ relative to other NP groups. 

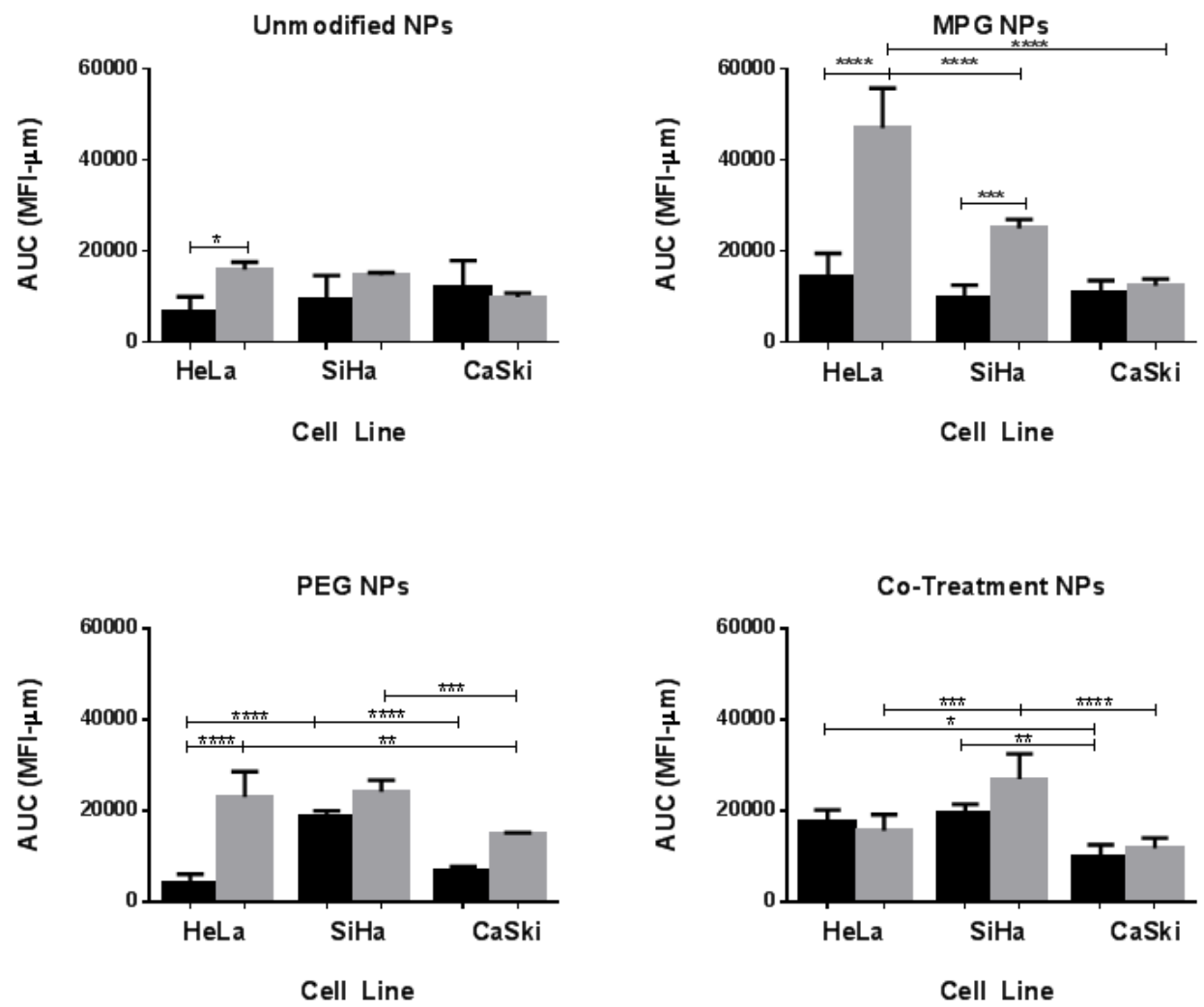

Figure 7. NP distribution represented as AUC for each tumor cell type (HeLa, $\mathrm{SiHa}$, or CaSki) as a function of NP treatment group, relative to spheroid type (LO, black and HD, gray). Values of all signifcant correlations, including each treatment group relative to unmodi- fed NPs, relative to other treatment groups, or relative to the same treatment group in a diferent spheroid type are given with degree of signifcance indicated $(* \mathrm{p}<0.01, * * \mathrm{p}<0.001, * * * \mathrm{p}<0.0001$, $* * * * \mathrm{p}<0.00001)$. Error bars: average \pm standard deviation $(\mathrm{n}=3)$

With these studies in mind, MPG NPs seem to be the most effective treatment group for the regularly-shaped HeLa tissue, whereas MPG, PEG, or MPG/PEG co-treatment may be used interchangeably for the regularly-shaped SiHa tissue. For larger, irregularly-shaped tissue, MPG 
and MPG/PEG or PEG and MPG/PEG NPs demonstrated promise for HeLa and SiHas respectively. Importantly, the MPG/PEG co-treatment consistently demonstrated NP penetration and distribution across all cell types relative to other NP groups, offering a potentially comparable treatment option relative to individual groups of surface-modified NPs. In future work, the NP co-treatment strategy may offer a significant increase in NP penetration and distribution across all cell and tumor types, if administered at the same individual NP concentrations as single NP treatments.

While the tumor microenvironment presents many challenges to achieving efficacious cargo delivery, our goal in these studies was to employ rational design to develop more therapeutically efficacious drug and gene delivery vehicles to overcome these challenges. One method used to enhance the delivery of active agents to cells is to directly complex or conjugate ligands to enhance tumor internalization and distribution. Notably, CPPs, such as Tat (the HIV transactivator protein) and MPG, have been widely used as drug and oligonucleotide conjugates to significantly enhance cellular internalization and localization $28,115,158,171-173$. In parallel work, many groups have conjugated ligands to delivery vehicles, such as NPs, to enhance the distribution of larger cargo within and to the tumor microenvironment $2,28,159,174-179$.

Another common strategy to enhance NP distribution, has been to modify the NP surface with different densities of, and molecular weight PEG molecules. In addition to reducing unwanted immune response and increasing the systemic half-life of NPs, this "PEGylation" 13,30,35,79 has enabled enhanced NP distribution in normal tissue or mucosal environments such as the female reproductive tract, gastrointestinal tract, and lung airways 8,13,28-30,35,39,79,136. Yet despite these contributions, there are few studies that have assessed how differences in tumor tissue morphology and cell origin, in addition to stealth or cell penetrating NP functionalization affect 
distribution. Our goal was to assess the impact these factors have on NP distribution through hypoxic/avascular regions of the heterogeneously vascularized tumor microenvironment in a cervical carcinoma model. In addition to being distal to vasculature and hence liable to receive less NPs/drugs, these regions are also usually resistant to cell-cycling drugs due to hypoxiainduced cell quiescence. The strategy proposed here is to enable more homogeneous and increased NP distribution into these regions, and for the NPs to remain long enough to affect cells once they resume cycling upon restored access to oxygen/nutrients.

It is well known that spheroid growth impacts cell proliferation, apoptosis, and necrotic/hypoxic core formation (e.g., ${ }^{180}$ ), with the outer layer (width $\sim 100 \mathrm{um}$ ) mostly proliferative, the middle layer hypoxic, and the inner core necrotic, as we have previously observed ${ }^{181,182}$. These conditions hold true independent of spheroid type ${ }^{181}$, as the diffusion of oxygen and nutrients into this system maintains cell populations with varying proliferative capability, contributing to chemoresistance for cell cycle-specific drugs, and replicates the diffusion limitations of bloodborne substances observed for tissue in vivo ${ }^{50,59,152,183}$. Accordingly, the bulk of NP uptake is expected to occur in the spheroid outer proliferative regions, as we have previously measured . Applying this knowledge to our studies, we suggest that highly proliferating regions at the tumor periphery, representing tissue adjacent to vasculature, combined with dormant/quiescent cells within the core, representing tissue distal from nanoparticle point of vascular extravasation, may form a gradient in the larger, irregularly-shaped (LO in our studies) tumor microenvironment, which may impede NP distribution ${ }^{184}$. Therefore we propose that the smaller HD spheroids, more closely mimicking regularly-shaped tumor tissue, likely have less structural variability and less necrotic tissue, perhaps contributing to the more uniform and increased distribution profiles observed. 
In addition to tumor size and morphology, we observed that NP distribution varied as a function of cell type. Indeed, likely as important as tumor size, cell origin plays a significant role in the development and diversity of the tumor microenvironment. Overall, we observed that both regularly and irregularly-shaped CaSki spheroid types seem to enable significantly less NP transport across all treatment groups when compared to SiHa and HeLa spheroids. We postulate that this difference in distribution may be due to ability of CaSki cells to form tighter intercellular junctions ${ }^{185}$, resulting in more regularly-shaped tissue with an intricate interstitial microenvironment. In such an environment, we may expect NP distribution to be hindered relative to less connected tissue. Further experimental investigation of junctional complex expression or integrity will be pursued in follow-up work, and is expected to provide more detailed information for optimizing targeting strategies. Furthermore, it is interesting to note that the MPG/PEG co-treatment NPs had consistently high NP distribution across all cell and spheroid types, particularly relative to other NP treatment groups in a given cell type. This suggests that the dual delivery strategy of MPG/PEG modified NPs is an efficacious treatment to penetrate and distribute throughout cervical tumors of different cell origins and varying degrees of tissue morphology.

One factor that may lead to similarities and differences seen between these different cell types is that they each possess HPV genomes of different subtypes. Other groups have studied immortalized cell lines derived from different tumor subtypes within the female reproductive tract to better understand phenotypic expression ${ }^{186-188}$. Additionally, the impact of incorporated HPV genomes on protein expression within 3D environments has been evaluated. It was discovered that HeLa cells, derived from an adenocarcinoma tumor subtype of the cervix, contain approximately 20-50 copies of integrated HPV-18, whereas SiHa cells, derived from 
grade II squamous cell carcinoma, and CaSki cells, derived from cervical epidermoid carcinomas, contain approximately 1-2 and 500 copies of integrated HPV-16, respectively ${ }^{186}$. Previous studies investigating the relationship between gap junctions, connexins, and tumor invasion as a function of HPV-related cervical cancer progression found that all three HPVassociated cervical cancer cell lines were poorly coupled and formed no appreciable gap junctions. Furthermore, both $\mathrm{SiHa}$ and HeLa cells were observed to have negligible levels of Connexin43 (an important transmembrane protein responsible for gap junction assembly), while very low levels were expressed in CaSki cells ${ }^{187}$. These findings support our observations that $\mathrm{SiHa}$ and HeLa cells are more permissive to transport than CaSki spheroids possibly due to these varying levels of gap junction proteins. Additionally, CaSki cells consistently formed more regularly-shaped spheroids, independent of spheroid formation method (HD or LO), indicating the impact of cell phenotype on tumor morphology.

In a separate study, the tumorigenicity of these cell lines was evaluated by observing the tumor forming ability post-injection in athymic mice; $\mathrm{SiHa}$ and $\mathrm{HeLa}$ cells were found to form tumors at lower cell density injections relative to CaSki cells. In the same study, $\mathrm{SiHa}$ and HeLa cells were shown to upregulate cancer-inducing cell-specific genes such as stem-cell markers, integrins, and epithelial to mesenchymal transition associated genes, and were found to be highly tumorigenic ${ }^{188}$. Taking these studies into consideration, it is likely that tumor spheroids formed from different cervical carcinoma cell lines generate varying tumor microenvironments and therefore affect NP penetration and distribution. Furthermore, it seems that capitalizing on the properties of a cationic CPP and neutral, hydrophilic ligand modified NPs may result in increased NP distribution through and accumulation in the tumor microenvironment, as shown here (Figs. 3-6; Tables 3, 4). 


\section{Efficacy of Surface-Modified PLGA Nanoparticles to Target Hypo-Vascularized Cervical Cancer Lesions.}

\section{A. CLINICAL SIGNIFICANCE AND BACKGROUND}

Cervical cancer is the second leading cause of cancer in women worldwide, contributing to more than 275,000 fatalities each year. Additionally, 528,000 new cases were estimated globally in 2012, accounting for almost $12 \%$ of female cancers ${ }^{189}$. Approximately $85 \%$ of cases occur in developing countries, with this burden attributed to inadequate screening combined with a lack of healthcare ${ }^{190,191}$. Early detection of pre-cancerous lesions or early stage cervical cancer during routine gynecological visits can lead to successful treatment, while undiagnosed disease can progress to harder-to-treat advanced stages.

Early-stage cervical cancer is associated with minimal symptoms and is typically discovered during physical or annual exams. Given the limited access that many women still have to screening and associated health care, in combination with the minimal symptoms associated with stage I disease, approximately $35 \%$ of cervical cancers are detected during stages II-III, with $10 \%$ detected during stage $\mathrm{IV}^{192,193}$. For cervical cancer cases that are undiagnosed during the early stages, late-stage treatment is limited to highly invasive procedures that include radiotherapy, chemotherapy, surgical intervention, or combined therapy. While radiotherapy and/or surgery is often used to treat early stage cervical cancer, chemotherapy is common for advanced stage cancers ${ }^{194,195}$.

A primary challenge facing chemotherapeutic delivery is poor distribution and corresponding efficacy, dependent on administration method ${ }^{196,197}$. Additional challenges include the lack of specificity attained with systemic administration, resulting in higher administrated doses to 
achieve efficacy, increased adverse effects, rapid active agent clearance, and the emergence of chemotherapeutic resistance. Localized delivery can circumvent some of these challenges; however, both systemic and localized delivery may fail to achieve distribution through the tumor tissue. Further, localized delivery to the female reproductive tract (FRT) can facilitate rapid vaginal clearance, resulting in difficulty maintaining efficacious concentrations of therapeutic agents.

To address these challenges, nanotherapeutic delivery vehicles have been designed to more safely and effectively promote active agent delivery directly to the tumor site and to enhance agent distribution within the tumor microenvironment, subsequent to penetration and uptake $^{198,199}$. Nanotherapeutic platforms, such as polymeric nanoparticles (NPs), provide a system in which encapsulation of chemotherapeutic agents can be achieved to prolong active agent stability and release, while reducing exposure to healthy, surrounding tissue. Furthermore, NP accumulation in tumor tissue can be promoted via the enhanced permeability and retention (EPR) effect. In particular, FDA-approved polymer-based platforms such as poly(lactic-coglycolic) acid (PLGA) NPs, have been engineered to reduce unwanted immunogenic responses, avoid organ clearance and tissue transport, target tumor tissue, penetrate the tumor insterstitium, improve uptake, and enhance therapeutic efficacy ${ }^{200,201}$. In addition, these platforms can also be tailored by altering the hydrophilic properties of the monomer subunits to reduce the burst release of chemotherapeutics or other encapsulated agents, and to provide sustained-delivery, corresponding with high uptake and antitumor effects in a variety of in vitro and in vivo cervical tumors $^{202}$.

The utilization of NP platforms to deliver chemotherapeutic agents has grown more prevalent in efforts to overcome the multi-drug resistant (MDR) properties of cervical cancers. Several 
studies have demonstrated enhanced efficacy of doxorubicin (Dox) by utilizing either inorganic or organic NP delivery systems ${ }^{198,203}$. A recent study investigated the use of folic acid conjugated quantum dots to deliver Dox to folate receptor-positive cervical cancer cells, demonstrating significant improvement in cellular uptake and therapeutic efficacy ${ }^{204}$. In a separate study utilizing boron nitride NPs loaded with Dox, it was found that these NPs only offered a modest therapeutic effect in KB cervical cancer cells relative to leukemia cell lines ${ }^{205}$. To understand the effect of Dox on cervical cancer cells with MDR, the intracellular distribution of Dox was investigated and it was found that the use of Dox conjugated NPs significantly changes these dynamics, resulting in a high level of Dox within cells, relative to free Dox. Furthermore, PLGA NPs incorporating Dox were modified with folic acid-polyethylene-glycol and studied in BALB/c mice that had been inoculated with KB cells. Administration of modified PLGA NPs significantly increased the efficacy of Dox, resulting in reduced tumor volume in vivo, relative to unmodified NPs and free Dox ${ }^{206}$. These promising results suggest that the use of NP delivery platforms may increase the therapeutic efficacy of Dox and other drug candidates, particularly for MDR cervical cancers.

Yet, one of the primary issues hindering the delivery and therapeutic efficacy of chemotherapeutics and other active agents is the inability of NPs to penetrate the tumor interstitium and, for some applications, undergo internalization by target cells. To overcome these transport-based challenges, NPs have been surface-modified with a variety of ligands to enhance tumor targeting and distribution. However, cellular internalization and tissue penetration are often diametrically opposed. In some cases, surface-modification with targeting or cell penetrating ligands, that contain cationic sequences, prevents the carrier from penetrating the tumor interstitium due to electrostatic interactions with the extracellular tumor matrix. In 
contrast, modification with ligands that facilitate penetration may provide broad distribution, but decrease internalization at the target cell surface ${ }^{28,207,208}$ Current NP designs often utilize one of these two approaches or iteratively determined ligand combinations to optimize delivery. Unfortunately, either case may result in similar ineffective therapy.

Previously, we investigated the effects of NP surface-modification with cell-penetrating peptides (CPPs), stealth ligands, and tumor targeting ligands (MPG (unabbreviated notation), PEG (polyethylene glycol), and Vimentin, respectively), on NP penetration and distribution within the hypo-vascularized tumor environment ${ }^{2}$. Nanoparticles modified with the CPP MPG exhibited the highest cellular internalization in multi-cellular, human cervical carcinoma (HeLa) 3D spheroids; however, internalization primarily occurred within the spheroid periphery, resulting in a modest $(<100 \mu \mathrm{m})$ tumor penetration depth. In contrast, PEG-modified NPs distributed more deeply into spheroids, but were less readily internalized by cells. More recently, we evaluated the penetration and distribution of PEG, MPG, and CPP-stealth (MPG/PEG) NP co-treatment strategies as a function of tumor morphology and size in 3D spheroid models of hypo-vascularized cervical cancer tissue $^{1}$. The differences in NP distribution as a function of surface-modification (unmodified, MPG, PEG, or MPG/PEG co-treatment), spheroid morphology, and cervical cancer type, were assessed in HeLa, cervical epithelial adenocarcinoma cells; CaSki, cervical epidermoid carcinoma cells; and SiHa, grade II cervical squamous cell carcinoma cells. From these studies, it was found that NP tumor distribution varied as a function of tumor size, cervical cancer type, and NP surface-modification. Interestingly, relative to unmodified NPs, MPG NPs exhibited enhanced distribution in HeLa spheroids, whereas PEG NPs were observed to have the greatest distribution in $\mathrm{SiHa}$ spheroids, for both small, homogeneously shaped and large, irregularly shaped tumors. Furthermore, it was noted that co-treatment of tumors with MPG- and 
PEG-modified NPs resulted in comparable distribution and tumor penetration across all tissue and tumor types, suggesting the potential for rational design to elicit the most efficacious treatment in vivo, in which the tumor architecture and composition are profoundly more complex than 3D spheroid cell cultures.

Building upon this previous work, the goal of this study was to determine the therapeutic efficacy imparted by these different delivery vehicles across different cervical tumor types. The chemotherapeutic agent, Dox, was incorporated into PLGA NPs with PEG, MPG, or PEG/MPG modifications to assess the differences in efficacy imparted as a function of surface modification, transport, and cell type. The NP efficacy in 2D cervical cancer monolayers and 3D tumor spheroids were assessed to characterize the different effects of treatment and to overcome the limitations of 2D testing. The chemotherapeutic Dox was used due to its application in a variety of chemotherapeutic regimens, including cervical cancer, and its ability to affect tumor cells at any stage of the cell cycle ${ }^{209,210}$. Moreover, while Dox has proven efficacious in killing both blood cancers and solid tumors, it is limited by the development of drug resistance, primarily caused by drug-resistant pumps such as P-glycoprotein (Pgp). Doxorubicin is also associated with acute side effects such as inducing cardiomyopathy, due to non-specific targeting. These limitations offer opportunities for engineered delivery systems to improve upon the clinical efficacy of Dox. 


\section{B. METHODS}

\section{Synthesis of Avidin-Palmitate Conjugates}

Avidin-palmitate was synthesized for subsequent conjugation to NPs as previously described 20,22,23,26. Briefly, $40 \mathrm{mg}$ of avidin (A9275, Sigma) was dissolved in $4.8 \mathrm{~mL}$ of $2 \%$ sodium deoxycholate $(\mathrm{NaDC})$ in phosphate buffered saline (PBS) warmed to $37^{\circ} \mathrm{C}$. Palmitic acid-NHS (PA-NHS, Sigma) was dissolved in $2 \% \mathrm{NaDC}$ to a final concentration of $1 \mathrm{mg} / \mathrm{mL}$ and sonicated until well-mixed. PA-NHS solution $(3.2 \mathrm{~mL})$ was added dropwise to the avidin NaDC solution, and reacted overnight at $37^{\circ} \mathrm{C}$. The following day, the reaction was dialyzed in $1200 \mathrm{~mL}$ of $0.15 \% \mathrm{NaDC}$ in PBS heated to $37^{\circ} \mathrm{C}$. Free PA-NHS was dialyzed overnight at $37^{\circ} \mathrm{C}$ using 3500 MWCO tubing to remove free palmitic acid. After overnight dialysis, the dialysis tubing contents were transferred to a storage vial and stored at $4^{\circ} \mathrm{C}$ until use.

\section{Nanoparticle Synthesis}

PLGA NPs encapsulating either the fluorophore Coumarin 6 (C6) or the chemotherapeutic doxorubicin (Dox) were synthesized as previously described ${ }^{1,2}$ to enable visualization via fluorescence microscopy and induce cell death, respectively. Briefly, NPs were synthesized using an oil-in-water $(\mathrm{o} / \mathrm{w})$ single emulsion technique ${ }^{1,2}$. Carboxyl-terminated poly(lactic coglycolic acid, PLGA) (0.55-0.75 dL/g, LACTEL $\left.^{\circledR}\right)$ was used to synthesize 100-200 mg batches. Encapsulant was dissolved in methylene chloride (DCM) overnight at a concentration of $15 \mu \mathrm{g}$

C6 per mg of PLGA or $100 \mu \mathrm{g}$ Dox per mg PLGA. The following day, the PLGA/encapsulant/DCM solution was added dropwise to a 5\% polyvinyl alcohol (PVA) solution of equal volume, vortexed, and sonicated. The resulting NPs were hardened in $0.3 \%$ PVA during solvent evaporation for $3 \mathrm{hr}$. 
Unmodified NPs were washed after hardening, and centrifuged at $4^{\circ} \mathrm{C}, 3$ times in deionized water (diH2O) to remove residual solvent. Nanoparticles were frozen, lyophilized, and stored at $20^{\circ} \mathrm{C}$ until use. A similar protocol was followed to synthesize MPG (3177 Da, GenScript) and PEG (5000 Da, Nanocs Inc.) surface-modified NPs, with the addition of avidin-palmitate (5 $\mathrm{mg} / \mathrm{mL}$ ) to the 5\% PVA solution. Surface-modified NPs were collected after the first wash, and incubated for 30 min. with biotinylated ligands at a molar ratio of 3:1 ligand:avidin in PBS. After the conjugation reaction, the NPs were washed two more times with $\mathrm{diH} 2 \mathrm{O}$ centrifugation, frozen, and lyophilized. All NPs were stored at $-20^{\circ} \mathrm{C}$ after synthesis.

\section{Nanoparticle Characterization \\ NP Physical Properties}

NP characterization confirmed the physical properties of NPs including unhydrated diameter, morphology, and surface charge. Scanning electron microscopy (SEM, Zeiss SUPRA 35VP) was utilized to verify unhydrated NP morphologies and diameters, measured using NIH ImageJ software. The NP zeta potentials were characterized using a Malvern Zetasizer (Zetasizer Nano ZS90).

\section{Controlled Release and Encapsulation}

To determine the loading and release properties of Dox NPs, encapsulation and controlled release experiments were conducted. To evaluate loading and encapsulation efficiency, approximately 3-5 mg of Dox NPs were suspended in $1 \mathrm{~mL}$ of DMSO for 30 minutes to dissolve the PLGA polymer matrix. Subsequently, the quantity of Dox loaded was determined by 
measuring the fluorescence $(480 \mathrm{~nm})$ and comparing to a calibrated Dox standard. The sustainedrelease properties of Dox NPs were determined by assessing controlled release in vitro. Dox NPs were resuspended in $1 \mathrm{~mL}$ of $1 \mathrm{X}$ PBS ( $\mathrm{pH} 7.4$ ) at a concentration of $2 \mathrm{mg} / \mathrm{mL}$ and incubated with gentle agitation at $37^{\circ} \mathrm{C}$. At fixed time points $(1,2,4,6,24,48,72,96 \mathrm{hr}, 1,2,3$, and 4 wk), samples were collected and the amount of Dox released from the NPs was quantified using absorbance (480nm) as described above.

\section{Three-Dimensional Cell Culture}

The human cervical carcinoma cell lines, SiHa, and CaSki (ATCC), were kindly provided by Dr. Alfred Jenson (University of Louisville), while the HeLa cell line was generously provided by Dr. Kenneth Palmer (University of Louisville). HeLa and SiHa cells were maintained in Minimum Essential Media (MEM) and CaSki cells were maintained in RPMI medium, both supplemented with $10 \%$ fetal bovine serum and $1 \%$ penicillin-streptomycin. All cells were kept in a humidified atmosphere of $5 \% \mathrm{CO} 2$ at $37^{\circ} \mathrm{C}$, and were grown to $80 \%$ confluence prior to tumor spheroid formation.

\section{Hanging Drop Tumor Spheroid Formation}

To form hanging drop spheroids, ultra-low attachment plates (\#4515, Corning) were utilized. Briefly, cells were trypsinized after reaching 80-90\% confluency and seeded at a density of 5,000 cells per well in $100 \mu \mathrm{L}$ culture medium. Care was taken to minimize pipette tip contact with

ultra-low attachment plate well walls. Spheroids were allowed to form for 5 days under the cell culture conditions described above. 


\section{Spheroid Characterization}

Spheroid morphology was characterized prior to NP administration. Briefly, spheroids were removed from culture plates and placed on imaging dishes (P35G-0-14-C, MatTek) in $25 \mu \mathrm{L}$ of culture medium to prevent drying. Spheroids were then imaged with an epifluorescent microscope (Axiovision 4, Zeiss) under transmitted light using a 10X objective.

\section{Flow Cytometry}

The association and internalization of NPs were determined using fluorescence-activated cell sorting (FACS). The four NP formulations: unmodified, PEG, MPG, and MPG/PEG cotreatment groups, were added to hanging drop spheroids for 1.5 or $24 \mathrm{hr}$. After incubation, the spheroids were collected in $2 \mathrm{~mL}$ eppendorf tubes and washed three times with PBS to remove unbound NPs. Trypsin-EDTA $(0.25 \%)$ was added to the spheroids for 5 min to dissociate the spheroids. Trypsinized cells were subsequently moved to FACS tubes, centrifuged, and resuspended in a FACS buffer solution containing $1 \%$ BSA and $0.1 \%$ sodium azide. From each sample, half the cells were moved to separate FACS tubes and kept on ice until analyzed (total associated samples). The remaining cells were exposed to $0.4 \%$ trypan blue for 5 min to quench extracellular fluorescence, washed twice in FACS buffer, and kept on ice until analyzed (internalized samples) [31]. All FACS tubes from both groups were analyzed using a BD LSRFortessa Flow Cytometer (BD Biosciences). Data were analyzed using FlowJo software (FlowJo Enterprise), and a minimum of 2,500 cells were analyzed per sample. 


\section{NP Efficacy in Cervical Cancer Monolayers and Spheroids}

\section{$2 D$ Chemotherapeutic Efficacy}

The chemotherapeutic efficacy of surface-modified Dox NPs in 2D monolayer cell cultures was determined via the MTT (3-(4, 5-dimethylthiazolyl-2)-2, 5-diphenyltetrazolium bromide) cell proliferation assay. Cervical carcinoma cells were seeded at a density of $1.5 \times 103$ cells per well and allowed to adhere overnight. Serial dilutions of surface-modified Dox NPs at a maximum NP concentration of $5 \mathrm{mg} / \mathrm{mL}$, were administered to cell monolayers. Untreated cells and cells treated with $10 \%$ DMSO were used as positive and negative controls of viability, respectively. Cells were then incubated under normal cell culture conditions for $24 \mathrm{hr}$. Following NP administration, media was discarded and replaced with $100 \mu \mathrm{L}$ of fresh PBS. Plates were then centrifuged at $300 \mathrm{rpm}$ for $5 \mathrm{~min}$, to remove free NPs. The PBS supernatant was discarded from the wells, and $10 \mu \mathrm{L}$ MTT reagent was added to each well in the absence of light. Plates were subsequently incubated under normal cell culture conditions for $4 \mathrm{hr}$. Following incubation, 50

$\mu \mathrm{L}$ lysis buffer $(10 \% \mathrm{SDS}$ in $0.01 \mathrm{M} \mathrm{HCl})$ was added to each well and placed in the incubator overnight. Sample absorbance was quantified $(570 \mathrm{~nm})$ the next day. 


\section{D Chemotherapeutic Efficacy}

To assess NP cytotoxicity in tumor spheroids, four different NP formulations were evaluated: unmodified, PEG, MPG, and MPG/PEG co-treatment groups. Once spheroid formation was achieved using the hanging drop method (5 days), fresh culture medium was added and the spheroids were transferred to 96-well opaque plates with $100 \mu \mathrm{L}$ media and NP treatments for 24 hr. For the NP co-treatment group, a half-dose of the MPG and PEG NPs were combined for a total NP dose of the desired concentration. After the $24 \mathrm{hr}$, the plated spheroids were removed from the incubator and equilibrated for $30 \mathrm{~min}$. Cell Titre-Glo $(100 \mu \mathrm{L})$ was added to each well, and the plate contents were mixed vigorously for $5 \mathrm{~min}$ on a reciprocating shaker. After mixing, the spheroids were incubated at room temperature for $25 \mathrm{~min}$ and luminescence was recorded on a BioTek Synergy HT luminometer.

\section{Statistical analysis}

Experiments were conducted each with a minimum sample size of $n=6$. Data were analyzed by using a two-way ANOVA applying Tukey's test $(* \mathrm{p}<0.01$, ** $\mathrm{p}<0.001$, *** $\mathrm{p}<0.0001$, **** $\mathrm{p}<0.00001)$. 


\title{
C. RESULTS
}

\author{
Nanoparticle Characterization
}

Size and Morphology

NP size and morphology were confirmed using SEM and ImageJ processing. Unhydrated NPs

demonstrated a spherical morphology, with diameters ranging between 160 to $180 \mathrm{~nm}{ }^{1,2}$. Hydrated NP surface charges were measured using a Zetasizer (Malvern). Unmodified NPs had a negative surface charge of $-14.8 \pm 1.2 \mathrm{mV}$; while PEG- and MPG-modified NPs measured $0.1 \pm$ 0.4 and $0.7 \pm 0.3 \mathrm{mV}$, respectively, validating surface ligand conjugation.

\section{Loading and Release}

Doxorubicin loading and encapsulation efficiency within MPG, PEG, and unmodified NPs were assessed (Table 1). MPG and PEG NPs exhibited significant increases in drug loading (65.9 \pm $2.3 \mu \mathrm{g} / \mathrm{mg}$ and $75.7 \pm 6.8 \mu \mathrm{g} / \mathrm{mg}$ ) and encapsulation efficiency (66\% and $76 \%$ ), relative to unmodified NPs $(28.9 \pm 1.5 \mu \mathrm{g} / \mathrm{mg}$ and $29 \%$ respectively).The in vitro release of Dox was characterized during a $24 \mathrm{hr}$ release study (Fig. 1). The unmodified NPs released approximately 95\% of the encapsulated Dox payload during the first $24 \mathrm{hr}$, while MPG and PEG NPs released $\sim 80-90 \%$ during the same duration. 
A

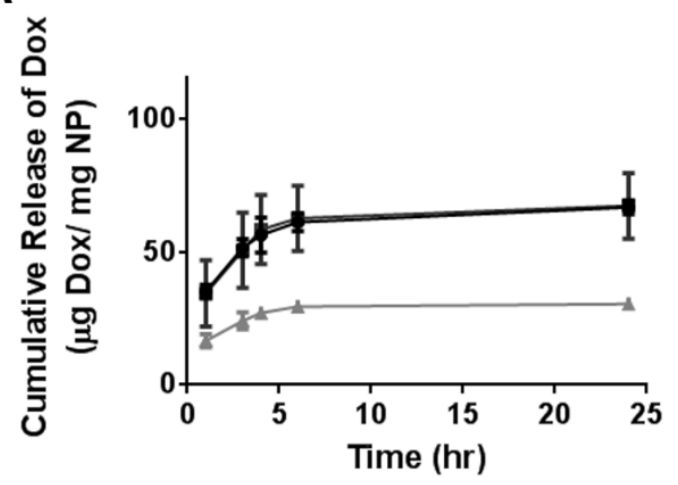

B

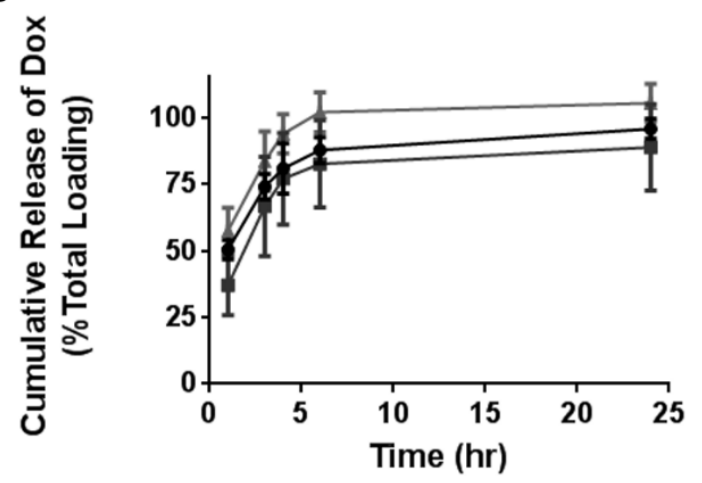

Figure 1. The cumulative release of Dox from unmodified (triangle), MPG (circle), and PEG (square) modified NPs, as a function of (A) NP mass and (B) percent total loading.

Table 1. Nanoparticle loading and encapsulation efficiency.

\begin{tabular}{|l|c|c|}
\hline \multicolumn{1}{|c|}{ NP Formulation } & Concentration $(\mu \mathrm{g}$ Dox per mg NP) & \% Encapsulation \\
\hline MPG & $65.9 \pm 2.3$ & $66 \%$ \\
\hline PEG & $75.7 \pm 6.8$ & $76 \%$ \\
\hline Unmodified & $28.9 \pm 1.5$ & $29 \%$ \\
\hline
\end{tabular}

Spheroid Morphology and Nanoparticle Association

Cervical tumor spheroids were formed from each HeLa, SiHa, and CaSki cell lines. Spheroid morphology was found to be dependent upon cervical tumor type (Fig. 2), as previously observed $^{1}$. Overall it was observed that HeLa cells demonstrated the most diffuse morphology, while CaSki cells formed the most dense tumor spheroids. 

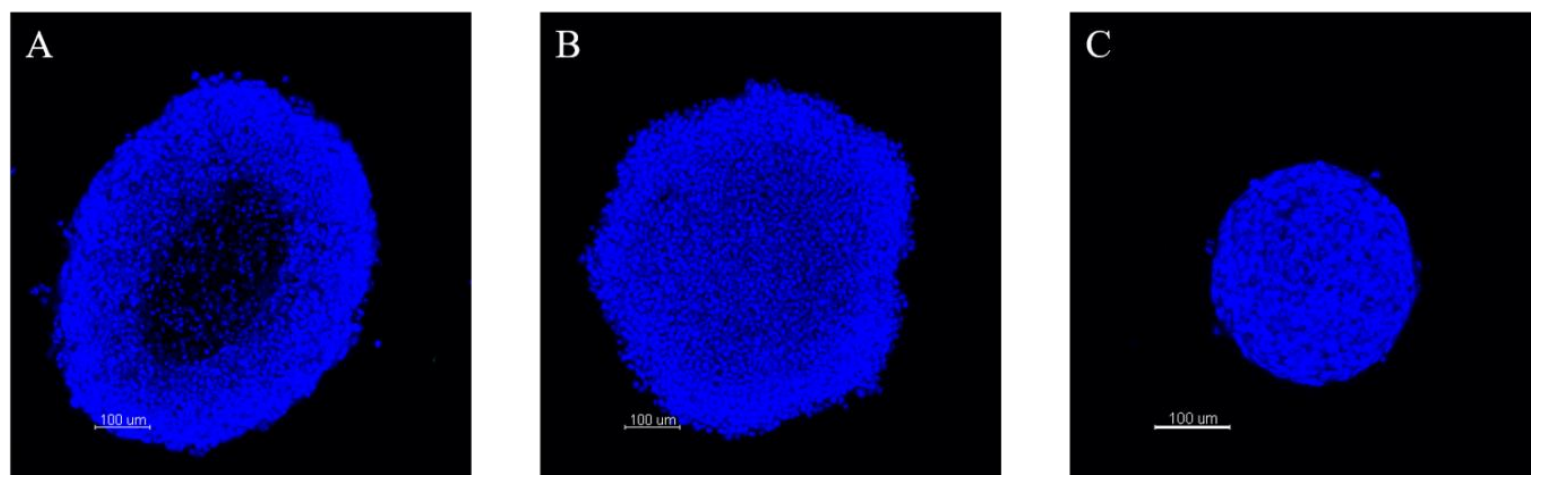

Figure 2. Fluorescent cross-sectional images of cervical tumor spheroids comprised of (A) HeLa, (B)

SiHa, and (C) CaSki cells stained with Hoechst dye.

To assess differences in NP localization as a function of spheroid morphology, NP association and internalization were evaluated in HeLa, SiHa, and CaSki cells, following incubation times of 1.5 and $24 \mathrm{hr}$ (Fig. 3). Differences in association (bound plus internalized), relative to internalization, varied as a function of both incubation time and cell type.

Within the HeLa spheroids (Fig. 2A), MPG NPs exhibited statistically significant increases in cellular association after $24 \mathrm{hr}$ administration, relative to unmodified and PEG NPs. Cotreatment NPs (MPG/PEG) had the second highest NP association although no statistical significance was observed relative to other NP groups. Similarly, MPG NPs demonstrated the highest cellular internalization in HeLa spheroids, although no statistically significant differences in internalization were observed relative to other NP treatment groups.

In the similarly sized, but less amorphous SiHa spheroids (Fig. 2B), unmodified NPs had the highest association followed by MPG NPs (Fig. 3). Moreover, all surface-modified NP groups exhibited trends of increased cellular internalization in SiHa spheroids relative to unmodified NPs; however, no statistical differences were observed between NP groups. 
In the smaller, densely structured CaSki spheroids, NP association and internalization were reduced across all treatment groups relative to that observed in HeLa and $\mathrm{SiHa}$ spheroids. MPG/PEG co-treatment NPs had statistically increased cellular association with respect to all NP groups. MPG NPs exhibited the second highest degree of NP association and PEG NPs were shown to have the lowest amount of NP association, relative to all other treatment groups. The MPG/PEG co-treatment NPs also had statistically higher levels of cell internalization in CaSki spheroids after $24 \mathrm{hr}$, relative to all other treatment groups. 

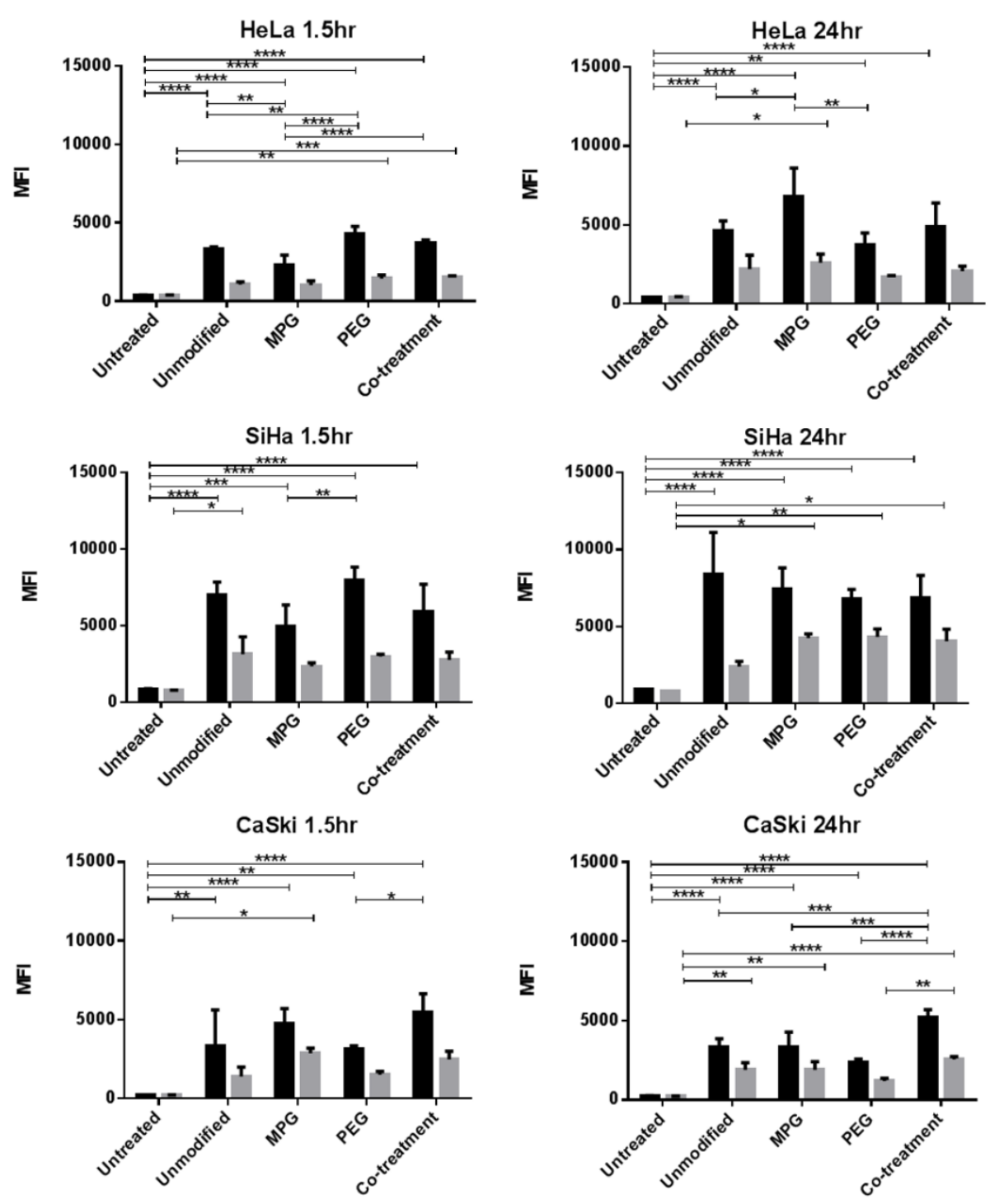

Figure 3. NP association (binding plus internalization, black) and internalization (grey) in 3D tumor spheroids after $1.5 \mathrm{hr}$ (left) and $24 \mathrm{hr}$ (right) administration. 


\section{Nanoparticle Efficacy against Cervical Cancer Monolayers and Spheroids}

To relate NP localization to chemotherapeutic efficacy, surface-modified NPs were administered to 2D cervical cell monolayers (Fig. 4) and 3D tumor spheroids (Fig. 5). The concentration at which 50\% of cell growth was inhibited (IC50) was evaluated for each NP treatment group as a function of Dox concentration (Figs. 4 and 5, Tables 2) and NP dose (data not shown). The efficacy imparted by NPs was found to vary as a function of cervical tumor and NP modification type. Figure 6 provides a summary of all IC50s across monolayers and spheroids, as a function of Dox and NP concentrations.

\section{NP Efficacy in HeLa Cells}

The efficacy of surface-modified Dox NPs was assessed relative to unmodified NPs and free Dox in different cervical cell type monolayers and spheroids, after $24 \mathrm{hr}$ administration. In HeLa spheroids, the MPG/PEG co-treatment NP group demonstrated a statistically significant increase in efficacy (IC50 $13.3 \pm 0.64 \mu \mathrm{M}$ ) relative to other NP groups. Free doxorubicin evinced a statistically significant increase in efficacy relative to NP treatment groups but statistically similar efficacy to the MPG/PEG co-treatment. Of the surface-modified groups, MPG NPs (IC50 $31.9 \pm 6.5 \mu \mathrm{M})$ performed slightly better, but statistically similar to PEG and unmodified NPs (34.7 $\pm 1.6 \mu \mathrm{M}$ and $33.4 \pm 5.5 \mu \mathrm{M}$, respectively), all of which had a statistically significant higher IC50 value relative to free Dox and the MPG/PEG NP co-treatment (IC50s 8.7 \pm 1.3 and $13.3 \pm 0.64 \mu \mathrm{M}$, respectively). Overall, the MPG/PEG NP co-treatment was the most efficacious NP group in HeLa spheroids. 
In HeLa monolayers unmodified NPs demonstrated the greatest efficacy, with a statistically significant decrease in IC50 $(20.3 \pm 2.2 \mu \mathrm{M})$ relative to surface-modified NP groups (MPG 28.6, PEG 39.5, and MPG/PEG 35.8 $\mu \mathrm{M})$ and free Dox $(25.4 \pm 3.9 \mu \mathrm{M})$. Similar to HeLa spheroids, PEG NPs were the least promising treatment group $(39.5 \pm 7.5 \mu \mathrm{M})$ in HeLa monolayers.

\section{NP Efficacy in SiHa Cells}

Relative to their modest efficacy in HeLa spheroids, unmodified NPs exhibited the greatest efficacy relative to other surface-modified NP groups in SiHa spheroids. Unmodified NPs exhibited a statistically lower IC50 (14.7 \pm 0.6$)$, relative to MPG $(30.2 \pm 1.2)$, PEG (23.3 \pm 1.1 $\mu \mathrm{M})$, and the MPG/PEG co-treatment NPs $(28.4 \pm 4.2 \mu \mathrm{M})$. Overall, MPG and the MPG/PEG co-treatment NPs were observed to be the least efficacious formulations in SiHa spheroids (30.2 \pm 1.2 and $28.4 \pm 4.2 \mu \mathrm{M}$, no statistical differences). All NPs provided modest efficacy against SiHa spheroids. Similar to HeLa spheroids, free Dox exhibited the lowest IC50 $(11.9 \pm 2.9 \mu \mathrm{M})$, with statistical significance observed relative to all NP groups.

In SiHa monolayers, PEG and co-treatment NP groups exhibited the strongest efficacy (IC50s $22.6 \pm 3.9 \mu \mathrm{M}$ and $27.2 \pm 3.3 \mu \mathrm{M}$ ) with statistically improved efficacy relative to free Dox (IC50 $42.3 \pm 13.7 \mu \mathrm{M})$. Overall, unmodified, PEG, and co-treatment NPs had similar IC50 values. Similar to 3D spheroids, MPG NPs (IC50 $34.1 \pm 8.5 \mu \mathrm{M}$ ) were the least efficacious NP formulation, demonstrating comparable efficacy to free Dox $(42.3 \pm 13.7 \mu \mathrm{M})$. While PEG and unmodified NPs showed the greatest efficacy in SiHa spheroids, PEG and co-treatment NPs performed most efficaciously in SiHa monolayers. 


\section{NP Efficacy in CaSki Cells}

Relative to the efficacy trends observed in HeLa and SiHa spheroids, the efficacy of NP groups within CaSki spheroids varied. Overall, PEG NPs were the most efficacious treatment group in CaSki spheroids (IC50 13.1 $\pm 1.1 \mu \mathrm{M}$ ), demonstrating a statistically significant improvement in efficacy relative to all other treatment groups. MPG NPs exhibited the second highest NP efficacy (IC50 22.5 $\pm 3.2 \mu \mathrm{M})$ followed by unmodified NPs $(25.38 \pm 2.6 \mu \mathrm{M})$ and the MPG/PEG co-treatment group $(25.6 \pm 3.7 \mu \mathrm{M})$. However, no statistical significance was observed between unmodified, MPG, and co-treatment NPs. As in HeLa and SiHa spheroids, free Dox exhibited the lowest IC50 $(1.8 \pm 0.5 \mu \mathrm{M})$, relative to all NP groups. Overall, PEG NPs and free Dox were most efficacious in CaSki spheroids.

Within CaSki monolayers, all NP groups demonstrated statistically significant improvements in efficacy, relative to free Dox. Within the NP groups, MPG/PEG co-treatment NPs were significantly more efficacious than MPG NPs (IC50s $18.1 \pm 2.3$ and $31.3 \pm 5.4 \mu \mathrm{M}$ ), and demonstrated similar efficacy to PEG and unmodified NPs. Furthermore, the co-treatment NP group was the most efficacious treatment, while MPG NPs were the least efficacious group, relative to free Dox. Overall, PEG NPs were the most effective treatment group in CaSki spheroids, while the MPG/PEG co-treatment NP group was the most promising formulation in CaSki monolayers. 

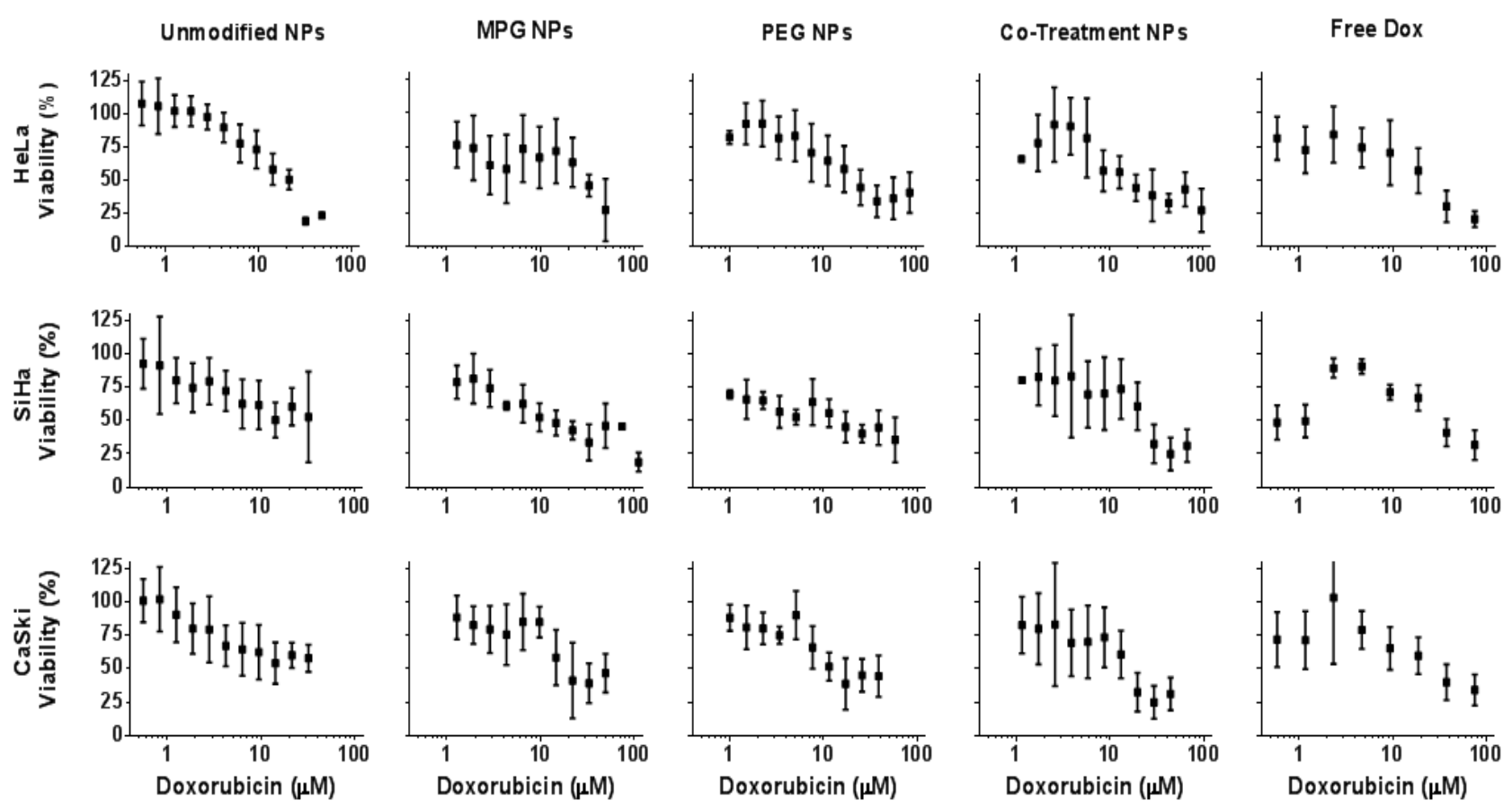

Figure 4. The cytotoxicity of surface-modified Dox NPs and free Dox in HeLa, SiHa, and CaSki cervical carcinoma monolayers after $24 \mathrm{hr}$ administration, as a function of Dox dose.
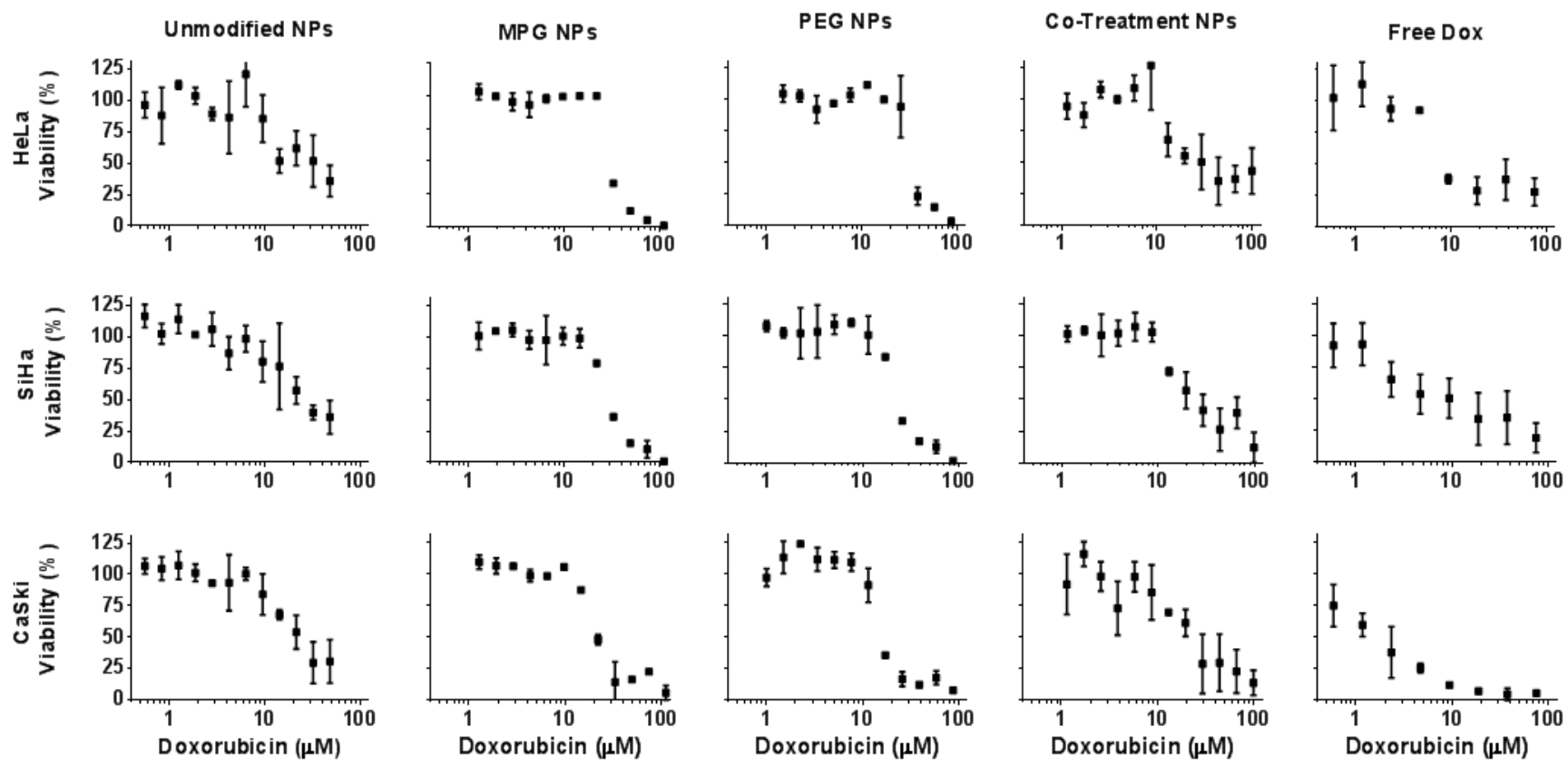

Figure 5. The cytotoxicity of surface-modified Dox NPs and free Dox in HeLa, SiHa, and CaSki cervical carcinoma 3D tumor spheroids after $24 \mathrm{hr}$ administration, as a function of Dox dose. 
A

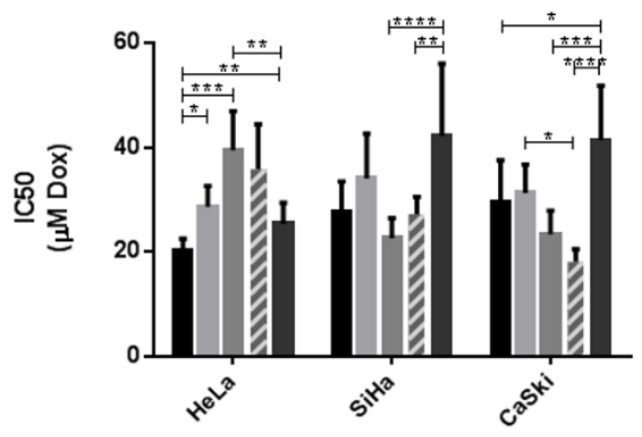

$\mathbf{C}$

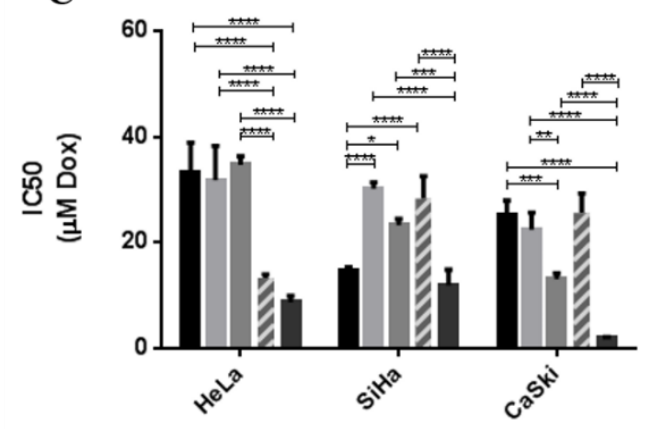

B

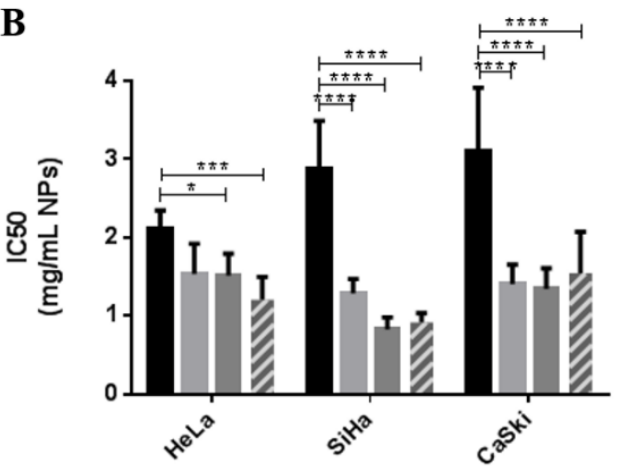

D

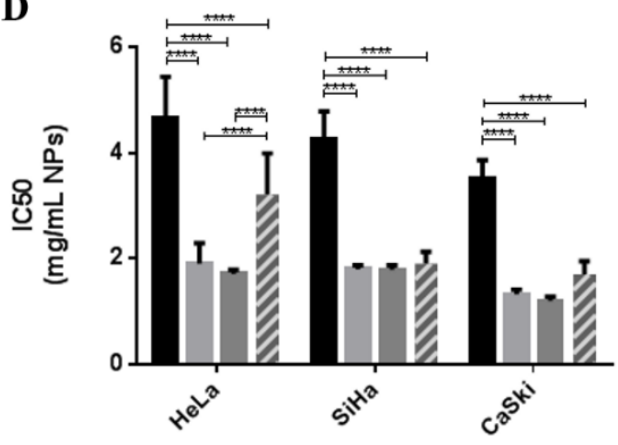

Unmodified NPs

MPG NPS

PEG NPS

VI Co-Treatment NPs

Free Dox

Figure 6. The IC50s of surface-modified NPs after $24 \mathrm{hr}$ administration in cervical carcinoma monolayers (top) and 3D tumor spheroids (bottom) as a function of Dox (A and C, $\mu \mathrm{M}$ ) and NP (B and $\mathrm{D}, \mathrm{mg} / \mathrm{mL}$ ) concentrations.

Table 2. IC50 Values of Surface-Modified Dox NPs and Free Dox in Cervical Cancer Monolayers and Tumor Spheroids Expressed in Terms of Dox Concentration.

\begin{tabular}{|c|l|c|c|c|c|c|}
\hline \multicolumn{2}{|c|}{$\begin{array}{c}\text { Cervical Cancer } \\
\text { Cell Model }\end{array}$} & \multicolumn{5}{|c|}{ Doxorubicin IC50 [ MM] } \\
\cline { 2 - 7 } & Free Dox & Unmodified NPs & MPG NPs & PEG NPs & Co-Treatment NPs \\
\hline \multirow{3}{*}{ Monolayers } & $\mathrm{HeLa}$ & $25.4 \pm 3.9$ & $20.3 \pm 2.2$ & $28.6 \pm 4.0$ & $39.5 \pm 7.5$ & $35.8 \pm 8.6$ \\
\cline { 2 - 7 } & $\mathrm{SiHa}$ & $42.3 \pm 13.7$ & $27.7 \pm 5.8$ & $34.1 \pm 8.5$ & $22.6 \pm 3.9$ & $27.2 \pm 3.3$ \\
\cline { 2 - 7 } & $\mathrm{CaSki}$ & $41.4 \pm 10.5$ & $29.7 \pm 7.9$ & $31.27 \pm 5.4$ & $23.4 \pm 4.5$ & $18.1 \pm 2.3$ \\
\hline \multirow{3}{*}{ Spheroids } & $\mathrm{HeLa}$ & $8.7 \pm 1.3$ & $33.4 \pm 5.5$ & $31.9 \pm 6.5$ & $34.7 \pm 1.6$ & $13.3 \pm 0.64$ \\
\cline { 2 - 7 } & $\mathrm{SiHa}$ & $11.9 \pm 2.9$ & $14.7 \pm 0.6$ & $30.2 \pm 1.2$ & $23.3 \pm 1.1$ & $28.4 \pm 4.2$ \\
\cline { 2 - 7 } & $\mathrm{CaSki}$ & $1.8 \pm 0.5$ & $25.38 \pm 2.6$ & $22.5 \pm 3.2$ & $13.1 \pm 1.1$ & $25.6 \pm 3.7$ \\
\hline
\end{tabular}




\section{Evaluation of NP Efficacy Based on NP Distribution}

Lastly, we assessed how the NP distribution in the 3D cell cultures of the various cell types, as observed in our previous work ${ }^{1}$, related to their efficacy measured in this study (Table 3 ).

Table 3. Comparison of NP efficacy (measured as IC50 in this study) to NP distribution (quantified as area-under-the-curve, AUC (MFI- $\mu \mathrm{m})$, in ${ }^{1}$ ) and maximum penetration (measured as mean fluorescence intensity (MFI) as a function of maximum penetration depth $(\mu \mathrm{m})$, in $\left.{ }^{1}\right)$ in 3D cell culture of various cervical cancer cell types.

\begin{tabular}{|c|c|c|c|c|c|}
\hline NP Measurement & Cell Type & Unmodified & MPG & PEG & Co-Treatment \\
\hline \multirow{2}{\text{DistributionshownasAUC}}{\begin{tabular}{c} 
(MFI- $\mu \mathrm{m})$ \\
\cline { 2 - 6 }
\end{tabular}} & $\mathrm{HeLa}$ & $15841 \pm 1637$ & $47018 \pm 8754$ & $23140 \pm 5531$ & $15657 \pm 3579$ \\
\cline { 2 - 6 } & $\mathrm{CaSki}$ & $14673 \pm 543$ & $24972 \pm 2020$ & $24237 \pm 2532$ & $26970 \pm 5574$ \\
\hline $\begin{array}{c}\text { Fluorescence Observed at } \\
\text { Max. Penetration Depth } \\
(\text { MFI, } \mu \mathrm{m})\end{array}$ & $\mathrm{HeLa}$ & $(99,85)$ & $(225,129)$ & $(217,82)$ & $(154,102)$ \\
\cline { 2 - 6 } & $\mathrm{SiHa}$ & $(123,61)$ & $(222,91)$ & $(135,64)$ & $(239,84)$ \\
\cline { 2 - 6 } & $\mathrm{CaSki}$ & $(83,52)$ & $(101,66)$ & $(238,78)$ & $(84,90)$ \\
\hline \multirow{3}{*}{$\begin{array}{c}\text { Efficacy shown as IC50 } \\
(\mu \mathrm{M})\end{array}$} & $\mathrm{HeLa}$ & $33.4 \pm 5.5$ & $31.9 \pm 6.5$ & $34.7 \pm 1.6$ & $13.3 \pm 0.64$ \\
\cline { 2 - 6 } & $\mathrm{SiHa}$ & $14.7 \pm 0.6$ & $30.2 \pm 1.2$ & $23.3 \pm 1.1$ & $28.4 \pm 4.2$ \\
\cline { 2 - 6 } & $\mathrm{CaSki}$ & $25.4 \pm 2.6$ & $22.5 \pm 3.2$ & $13.1 \pm 1.1$ & $25.6 \pm 3.7$ \\
\hline
\end{tabular}




\section{DISCUSSION}

In previous work we investigated the effect of surface modification on NP penetration and cell internalization in cervical tumor tissue ${ }^{2}$. In both HeLa monolayers and spheroids, nanoparticles modified with the CPP MPG exhibited the highest cellular internalization. However, internalization primarily occurred within the spheroid periphery, resulting in a modest $(<100$ $\mu \mathrm{m})$ tumor penetration depth. In contrast, PEG-modified NPs distributed most deeply into spheroids, but were less readily internalized by cells, underscoring the dichotomous transport properties of NPs modified with a CPP, relative to a neutral "stealth" ligand.

To expand upon this work, we subsequently evaluated surface-modified NP distribution through cervical tumors, as a function of cervical tumor type and morphology ${ }^{1}$. We found that distribution varied as a function of cervical cancer type and tissue morphology, in addition to NP surface-modification. In small, homogeneously shaped avascular tumors, enhanced distribution was observed for MPG and PEG NPs in HeLa, and for all surface-modified NPs in SiHa spheroids, relative to unmodified NPs. In larger, more irregularly-shaped spheroids, the greatest distribution was observed for MPG and MPG/PEG co-treatment NPs in HeLa, and for PEG and MPG/PEG co-treatment NPs in SiHa spheroids ${ }^{1}$. In contrast, small, densely structured CaSki spheroids exhibited modest distribution across all NP groups. Overall, for a given tumor type, surface modifications were found to benefit tumor distribution, relative to utilization of unmodified NPs. These results suggested that co-treatment strategies may overcome the obstacles associated with NP tumor penetration and distribution, and may influence the design of delivery platforms for cancer therapy, especially for tumors expected to present both regularlyand irregularly-shaped tissue regions. 
Given these observations, the aim of this study was to relate the transport properties of surfacemodified NPs to therapeutic efficacy as a function of cervical cancer type. To evaluate this, the efficacy of unmodified and surface-modified NPs, encapsulating the chemotherapeutic Dox, was assessed in three cervical carcinoma cell lines (HeLa, $\mathrm{SiHa}$, and CaSki), grown as 3D spheroids to represent tumor lesions with a hypervascularized periphery and hypovascularized interior. We selected Dox, as it is a commonly used chemotherapeutic; however, its efficacy against cervical tumors has been limited due to the MDR of several cervical cancer types, including $\mathrm{HeLa}$, SiHa, and CaSki lines. We hypothesized that the use of NPs may significantly increase the therapeutic efficacy of Dox, by increasing intracellular Dox accumulation, as MDR cells are prone to have more drug efflux due to an increased presence of p-glycoprotein pumps ${ }^{198,211}$. Moreover, the mechanism of action of Dox intercalation with DNA, inducing topoisomerase II inhibition and cellular DNA damage, makes it a model drug to represent active agents that must internalize to elicit therapeutic effect.

While the purpose of this study was to assess the effect of NP surface-functionalization on cervical tumor cytotoxicity, drug loading and release have integral roles in achieving therapeutic effect. The modest encapsulation efficiencies of unmodified NPs (29\%), relative to MPG- and PEG-modified NPs (66 and 76\% of Dox, respectively, Table 1), resulted in variations of release quantity with respect to time. Unmodified NPs released $28.3 \pm 2.1 \mu \mathrm{g}$ Dox/mg NP within $24 \mathrm{hr}$, while MPG and PEG NPs exhibited significantly higher release $(66.5 \pm 2.6$ and $67.2 \pm 12 \mu \mathrm{g}$ Dox/mg NP, respectively) during the same duration (Fig. 1).

When compared as a function of Dox concentration, relative to NP dose, these loading and release differences had an impact on therapeutic efficacy (measured as the IC50). For example, unmodified NPs, which were the most efficacious in SiHa spheroids $(14.7 \pm 0.6 \mu \mathrm{M}$, Fig. 6 , 
Table 2) as a function of Dox concentration, required a higher NP dose (3-5 mg/mL, data not shown), relative to surface-modified NPs, to achieve efficacy across all tumor types (HeLa, SiHa, CaSki). Furthermore, although they demonstrated modest efficacy relative to other NP treatment groups, unmodified NPs were still more efficacious than free Dox in CaSki and HeLa monolayers. Therefore, discrepancies in therapeutic efficacy as a function of NP dose relative to Dox dose, may be attributed to the approximately 2-fold lower drug loading and corresponding lower release observed with unmodified NPs. Moreover, surface-modified NPs may release less encapsulant during the fabrication process, contributing to overall higher loading postfabrication. These observations confirm the need to quantify therapeutic efficacy as a function of both drug release and NP dosing.

In addition to cytotoxicity differences based on NP loading and release profiles, the therapeutic efficacy of surface-modified NPs varied as function of cell type and NP surface modification. While unmodified NPs were most efficacious in SiHa spheroids as a function of Dox concentration, MPG/PEG co-treatment and PEG NPs were the most efficacious groups in HeLa and CaSki spheroids, respectively. Notably, in the more densely packed CaSki spheroids, PEG NPs were the most efficacious; however, all NP groups were less efficacious than free Dox alone. These results suggest specific surface modifications, dependent on tumor type, may elicit similar therapeutic effect as free Dox. Together, these results are consistent with the fact that tumor cell type and the associated microenvironment significantly impact therapeutic efficacy.

Together with the effects of the tumor microenvironment alone, the effect of active agent on the tumor microenvironment has been explored to better understand poor efficacy. Previous work investigated the effect of free Dox in tumor spheroids, and found that HeLa spheroids retained morphology and proliferated, even after treatment with a range of Dox concentrations $(0-40$ 
$\mu \mathrm{M})^{212}$. One proposed reason was that the extracellular matrix within the spheroid microenvironment remains intact following cell death ${ }^{212}$. Specifically for Dox, it was observed that efficacious levels of Dox failed to be internalized within $24 \mathrm{hr}$, resulting in reduced cytotoxic effect. Considering this, the use of specific NP modifications, while providing similar or less efficaciousness dependent on tumor type, may provide benefit within the real tumor microenvironment, by minimizing drug toxicity to surrounding tissue, providing longer exposure, and enhanced distribution. Moreover, NP diffusion, relative to free Dox delivery, may promote NP immobilization in the tumor matrix, prolonging the effects of localized cell death while reducing overall systemic cytotoxicity.

In addition to drug-specific effects on the tumor microenvironment, the effect of tumor density has been determined to play a role in the pharmacokinetics of chemotherapeutics within solid tumors. In particular, tumors that have a reduced tissue density have increased molecule penetration $^{196,213-215}$. Similarly, in our previous studies, we observed that the decreased density of HeLa and $\mathrm{SiHa}$ spheroids, relative to CaSki spheroids correlated to increased levels of NP penetration $^{1}$. Additionally, the difference in levels of HPV genome incorporation and oncogenic E6/E7 expression between cervical tumor cells underscores the variations in spheroid growth and permeability as a function of cell type ${ }^{186,216}$. In studies by Hoppe-Seyler and group, it was found that the expression of rate-limiting glycolytic enzymes was significantly influenced by incorporation of HPV oncogenes E6/E7, leading to enhanced rates of aerobic glycolysis ${ }^{217}$. The investigation revealed the presence of these enzymes was dramatically elevated in HeLa cells, relative to $\mathrm{CaSki}$ and $\mathrm{SiHa}$. This propensity for HeLa cells to have enhanced rates of aerobic glycolysis could significantly impact the gradient of proliferating cells in the tumor microenvironment. Another study by the same group found that under hypoxic conditions, HPV- 
positive cells can undergo a reversible state of dormancy and avoid senescence ${ }^{218}$. This effect could also contribute to variations in regions of proliferating versus dormant cells and impact the microenvironment at regions of gradient transition within the tissue.

As well as the effects observed from oncogenic expression, other work has evaluated the role of gap junction formation in immortalized cervical cancer cell progression. These studies revealed that both HeLa and $\mathrm{SiHa}$ cells have negligible levels of Connexin 43 relative to CaSki cells, and therefore exhibit lower levels of gap junction formation ${ }^{187}$. This is in agreement with our current and previous work, in which we observed that CaSki cells consistently form compact, dense spheroids that are less permissive to NP transport ${ }^{1}$. The dependency of pharmacokinetics on tumor spheroid intercellular interactions and cell density may also contribute to the increase in efficacy of MPG/PEG co-treatment NPs in HeLa spheroids relative to SiHa and CaSki spheroids. The more permeable nature of HeLa spheroids may be favorable for both MPG and PEG surface-modified NPs to more easily diffuse through the spheroid. Alternatively, as in our previous work ${ }^{1,2}$, the different distribution profiles of MPG and PEG NPs, may benefit from coadministration strategies (here, MPG/PEG co-treatment) that target tumor cells in different tumor regions (MPG: periphery, PEG: core). In contrast, the increased efficacy of unmodified NPs in SiHa spheroids and PEG NPs in CaSki spheroids, relative to the co-treatment in the less amorphous spheroids, suggests the advantage modification may have in enabling the delivery of therapeutically effective levels of active agent in tumors of increasing variability and unique microenvironment conditions.

Another aspect of NP efficacy that is often overlooked, is the relationship between transport and NP association and internalization at their target site. Thus, increased NP distribution and penetration into tumor tissue may not necessarily correlate with improved efficacy (Table 3). 
While it has been previously established that Dox must be internalized to exert therapeutic effect, NPs add an additional layer of complexity (yet potential enhancement) to the delivery process. To relate these properties, total NP association (binding and internalization) and internalization were assessed, dependent on cell and surface-modification type.

In HeLa spheroids, MPG NPs had the highest levels of cell association (bound extracellularly as well as internalized, $6823 \mathrm{MFI}$ ) relative to all other NP groups after $24 \mathrm{hr}$ administration, with statistical significance observed relative to unmodified and PEG NPs (Fig. 3). Although there was no statistical difference between NP groups for cell internalization (Fig. 3), the association results suggest that MPG NPs may be interacting with the extracellular components of the tumor periphery where proliferating cells are present, while PEG NPs encounter fewer interactions with the tumor microenvironment and diffuse more freely through the spheroid. This transport behavior, observed also in our previous work ${ }^{1}$, may support the increase in efficacy of the MPG/PEG co-treatment NPs observed, particularly in more amorphously-shaped HeLa spheroids.

In SiHa spheroids, there were no statistical differences between NP groups for cell association after 24 hr. (Fig. 3). Similarly, while all surface-modified NPs exhibited trends of improved cell internalization in $\mathrm{SiHa}$ spheroids relative to unmodified NPs, no statistical significance was observed (Fig. 3). Here we hypothesize that even slight increases in cell association, seen with unmodified NPs, may correlate with the increase in therapeutic efficacy of unmodified NPs, relative to surface-modified NPs in SiHa spheroids. These results support the idea that tumor distribution must be considered to effectively deliver therapeutic levels of Dox within the TME.

In CaSki spheroids, the MPG/PEG co-treatment group demonstrated higher cell association relative to all other NP groups and higher levels of cell internalization relative to PEG NPs (Fig. 
3). Interestingly, the MPG/PEG co-treatment group was the most efficacious in CaSki monolayers (Figs. 4-6, Table 2) but the least efficacious NP group in CaSki spheroids. These results substantiate previously observed trends that the bulk of NP uptake occurs along the tumor periphery (for hypovascularized lesions) and for tissue proximal to the vasculature (for vascularized tumors). When delivering therapeutic agents, it is important to consider that an adequate amount of NPs and associated therapeutic payload may not be internalized in cells distal from the vascular supply. These results are consistent with our previous

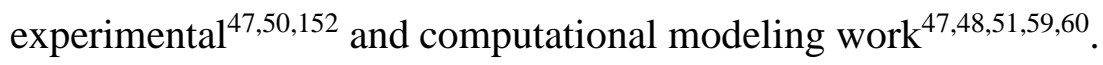

While the effects of NP binding and penetration can be observed in tumor spheroids, the significance of NP penetration is even more pronounced for treatment in hypoxic tumor conditions, where hypoxia is often associated with elevated levels of chemoresistance ${ }^{215}$. Our previous work ${ }^{50,151}$ has confirmed that spheroid growth impacts cell proliferation, apoptosis, and necrotic/hypoxic core formation, consistent with previous studies ${ }^{180}$. Moreover, these studies have noted that the outer layer (width $\sim 100 \mu \mathrm{m}$ ) is mostly proliferative, the middle layer hypoxic, and the inner core necrotic. These microstructural differences, in which the inner tumor layer is comprised of hypoxic cells, may contribute to decreased cell sensitivity to Dox, as hypoxic cells would be quiescent and thus avoid damage by the drug action during the cell cycle. Transient hypoxia has been shown to activate and upregulate genes encoding P-glycoprotein and dihydrofolate reductase, leading to resistance to drug substrates that are reliant on these molecules. Furthermore, tumor hypoxia has been shown to disrupt protein folding in the endoplasmic reticulum due to associated glucose deprivation ${ }^{219-221}$. This protein misfolding induces drug resistance to topoisomerase II-targeted drugs, such as Dox. In addition, Dox is known to react in the presence of oxygen and reduce to a superoxide which contributes to 
cytotoxicity. Within the hypoxic regions of the tumor, these effects may be attenuated due to the decrease in free radical production. To overcome this increase in drug resistance, an effective delivery strategy would require NPs to adequately diffuse to the inner portion of the tumor and undergo subsequent cell internalization. As the results suggest, the ability of NPs to deliver an adequate therapeutic payload to inner portions of a tumor are highly dependent on tumor cell type and NP surface modification. This suggests that an effective drug delivery strategy may be possible by utilizing various NP surface modification ligands if the disease cell origin is known.

The study presented here utilizes both single and co-treatment surface-modified NP delivery strategies to assess the efficacy of the chemotherapeutic Dox against hypovascularized cervical tumors of different disease origins. The results highlight the dependency of therapeutic efficacy on tumor cell type and the associated microenvironment. This offers the possibility to tailor the NP-mediated delivery strategy to maximize therapeutic efficacy based on patient-specific tumor tissue characteristics. 


\section{CONCLUSIONS}

We have presented single and dual NP delivery strategies, that utilize NPs modified with stealth and/or cell penetration ligands, to target hypo-vascularized tumor tissue. The results of these studies suggest the potential benefit that combination treatment, e.g., in which PEG NPs are used to achieve penetration into tissue distal from vasculature, while MPG NPs target proliferative regions close to blood vessels, may have in cervical cancer therapy. Interestingly, while hybrid MPG/PEG NPs had similarly high uptake as MPG after $24 \mathrm{hr}$ in tumor spheroids, lower uptake and penetration depth was observed, relative to either MPG or PEG NPs alone. In this case, internalization and diffusion may be competitive, thus inhibiting NP uptake and transport. These results highlight the well-known need to balance NP properties to achieve both adequate internalization and penetration through the tumor microenvironment.

In contrast with utilizing NPs that are co-modified with stealth and cell penetrating ligands, subsequent results suggest that a co-treatment strategy may provide the greatest intratumoral penetration across a variety of cervical cancer disease/cell origins. For smaller, more regularlyshaped tumor tissue, all surface-modified groups had similar distribution trends, relative to unmodified NPs. Moreover, for both smaller regularly- and larger irregularly-shaped tumors, if MPG/PEG NPs are administered at the same concentration as the modified NPs alone, the cotreatment has the potential to offer an increase in NP penetration and distribution in all cell and tumor types. This provides hope that a NP co-treatment strategy may overcome the obstacles associated with NP tumor penetration and may influence the design of delivery platforms for cancer therapy, especially since tumors are expected to present both regularly- and irregularlyshaped tissue regions. 
Subsequent therapeutic efficacy studies highlight the importance of understanding the underlying cancer origin when treating cervical cancer. The work presented here suggests that for an adequate amount of drug payload to be achieved intratumorally, the NP drug delivery platform would be required to diffuse throughout the tumor and undergo subsequent cell internalization. These results underscore the dependency of NP tumor diffusion and cytotoxicity on cell and tumor type. As observed from this work, utilization of surface-modifications have the potential to increase NP transport throughout the tumor interstitium and improve cell internalization. Thus, this strategy offers a powerful drug delivery platform if disease origin is known. Long-term, the knowledge gained from these studies may offer guidance regarding the most efficacious strategies to treat tumors of varying cervical cancer origin and stages of tumor progression. 


\section{References}

1 Sims, L. B., Huss, M. K., Frieboes, H. B. \& Steinbach-Rankins, J. M. Distribution of PLGA-modified nanoparticles in 3D cell culture models of hypo-vascularized tumor tissue. J Nanobiotechnology 15, 67, doi:10.1186/s12951-017-0298-x (2017).

2 Sims, L. B., Curtis, L. T., Frieboes, H. B. \& Steinbach-Rankins, J. M. Enhanced uptake and transport of PLGA-modified nanoparticles in cervical cancer. J Nanobiotechnology 14, 33, doi:10.1186/s12951-016-0185-x (2016).

3 Katz, D. F., Yuan, A. \& Gao, Y. Vaginal drug distribution modeling. Adv Drug Deliv Rev 92, 2-13, doi:10.1016/j.addr.2015.04.017 (2015).

4 Lai, S. K., Wang, Y. Y., Wirtz, D. \& Hanes, J. Micro- and macrorheology of mucus. Adv Drug Deliv Rev 61, 86-100, doi:10.1016/j.addr.2008.09.012 (2009).

5 Gao, Y. \& Katz, D. F. Multicompartmental pharmacokinetic model of tenofovir delivery by a vaginal gel. PLoS One 8, e74404, doi:10.1371/journal.pone.0074404 (2013).

6 Acarturk, F. Mucoadhesive vaginal drug delivery systems. Recent Pat Drug Deliv Formul 3, 193205 (2009).

7 Caramella, C. M., Rossi, S., Ferrari, F., Bonferoni, M. C. \& Sandri, G. Mucoadhesive and thermogelling systems for vaginal drug delivery. Adv Drug Deliv Rev 92, 39-52, doi:10.1016/j.addr.2015.02.001 (2015).

8 Ensign, L. M., Cone, R. \& Hanes, J. Nanoparticle-based drug delivery to the vagina: a review. J Control Release 190, 500-514, doi:10.1016/j.jconrel.2014.04.033 (2014).

9 Mallipeddi, R. \& Rohan, L. C. Nanoparticle-based vaginal drug delivery systems for HIV prevention. Expert Opin Drug Deliv 7, 37-48, doi:10.1517/17425240903338055 (2010).

10 Steinbach, J. M. Protein and oligonucleotide delivery systems for vaginal microbicides against viral STIs. Cell Mol Life Sci 72, 469-503, doi:10.1007/s00018-014-1756-3 (2015).

11 Whaley, K. J., Hanes, J., Shattock, R., Cone, R. A. \& Friend, D. R. Novel approaches to vaginal delivery and safety of microbicides: biopharmaceuticals, nanoparticles, and vaccines. Antiviral Res 88 Suppl 1, S55-66, doi:10.1016/j.antiviral.2010.09.006 (2010).

$12 \mathrm{Cu}, \mathrm{Y}$., Booth, C. J. \& Saltzman, W. M. In vivo distribution of surface-modified PLGA nanoparticles following intravaginal delivery. Journal of Controlled Release 156, 258-264, doi:10.1016/j.jconrel.2011.06.036 (2011).

$13 \mathrm{Cu}$, Y. \& Saltzman, W. M. Controlled surface modification with poly(ethylene)glycol enhances diffusion of PLGA nanoparticles in human cervical mucus. Mol Pharm 6, 173-181, doi:10.1021/mp8001254 (2009).

$14 \mathrm{Cu}, \mathrm{Y}$. \& Saltzman, W. M. Drug delivery: Stealth particles give mucus the slip. Nat Mater 8, 11-13, doi:10.1038/nmat2347 (2009).

15 das Neves, J. et al. Biodistribution and pharmacokinetics of dapivirine-loaded nanoparticles after vaginal delivery in mice. Pharm Res 31, 1834-1845, doi:10.1007/s11095-013-1287-x (2014).

16 das Neves, J. et al. In vitro and ex vivo evaluation of polymeric nanoparticles for vaginal and rectal delivery of the anti-HIV drug dapivirine. Mol Pharm 10, 2793-2807, doi:10.1021/mp4002365 (2013).

17 das Neves, J., Nunes, R., Machado, A. \& Sarmento, B. Polymer-based nanocarriers for vaginal drug delivery. Adv Drug Deliv Rev 92, 53-70, doi:10.1016/j.addr.2014.12.004 (2015).

18 das Neves, J. et al. Interactions of microbicide nanoparticles with a simulated vaginal fluid. Mol Pharm 9, 3347-3356, doi:10.1021/mp300408m (2012).

19 El-Hammadi, M. M. \& Arias, J. L. Nano-sized platforms for vaginal drug delivery. Curr Pharm Des 21, 1633-1644 (2015). 
Ensign, L. M. et al. Pretreatment of human cervicovaginal mucus with pluronic F127 enhances nanoparticle penetration without compromising mucus barrier properties to herpes simplex virus. Biomacromolecules 15, 4403-4409, doi:10.1021/bm501419z (2014).

21 Ensign, L. M. et al. Mucus-penetrating nanoparticles for vaginal drug delivery protect against herpes simplex virus. Sci Transl Med 4, 138ra179, doi:10.1126/scitransImed.3003453 (2012).

22 Lai, S. K. et al. Rapid transport of large polymeric nanoparticles in fresh undiluted human mucus. Proc Natl Acad Sci U S A 104, 1482-1487, doi:10.1073/pnas.0608611104 (2007).

23 Lai, S. K., Wang, Y. Y., Hida, K., Cone, R. \& Hanes, J. Nanoparticles reveal that human cervicovaginal mucus is riddled with pores larger than viruses. Proc Natl Acad Sci U S A 107, 598603, doi:10.1073/pnas.0911748107 (2010).

24 Lembo, D. et al. Encapsulation of Acyclovir in new carboxylated cyclodextrin-based nanosponges improves the agent's antiviral efficacy. Int J Pharm 443, 262-272, doi:10.1016/j.ijpharm.2012.12.031 (2013).

$25 \mathrm{Li}, \mathrm{C}$. et al. Sulfonate-modified phenylboronic acid-rich nanoparticles as a novel mucoadhesive drug delivery system for vaginal administration of protein therapeutics: improved stability, mucin-dependent release and effective intravaginal placement. Int J Nanomedicine 11, 59175930, doi:10.2147/IJN.S113658 (2016).

26 Maisel, K. et al. Nanoparticles coated with high molecular weight PEG penetrate mucus and provide uniform vaginal and colorectal distribution in vivo. Nanomedicine (Lond) 11, 1337-1343, doi:10.2217/nnm-2016-0047 (2016).

27 Frohlich, E. \& Roblegg, E. Mucus as barrier for drug delivery by nanoparticles. J Nanosci Nanotechnol 14, 126-136 (2014).

28 Steinbach, J. M., Seo, Y. E. \& Saltzman, W. M. Cell penetrating peptide-modified poly(lactic-coglycolic acid) nanoparticles with enhanced cell internalization. Acta Biomater 30, 49-61, doi:10.1016/j.actbio.2015.11.029 (2016).

29 Suk, J. S., Xu, Q., Kim, N., Hanes, J. \& Ensign, L. M. PEGylation as a strategy for improving nanoparticle-based drug and gene delivery. Adv Drug Deliv Rev 99, 28-51, doi:10.1016/j.addr.2015.09.012 (2016).

30 Tang, B. C. et al. Biodegradable polymer nanoparticles that rapidly penetrate the human mucus barrier. Proc Natl Acad Sci U S A 106, 19268-19273, doi:10.1073/pnas.0905998106 (2009).

31 Wang, Y. Y. et al. Mucoadhesive nanoparticles may disrupt the protective human mucus barrier by altering its microstructure. PLoS One 6, e21547, doi:10.1371/journal.pone.0021547 (2011).

32 Wong, T. W., Dhanawat, M. \& Rathbone, M. J. Vaginal drug delivery: strategies and concerns in polymeric nanoparticle development. Expert Opin Drug Deliv 11, 1419-1434, doi:10.1517/17425247.2014.924499 (2014).

33 Woodrow, K. A. et al. Intravaginal gene silencing using biodegradable polymer nanoparticles densely loaded with small-interfering RNA. Nat Mater 8, 526-533, doi:10.1038/nmat2444 (2009).

$34 \mathrm{Xu}, \mathrm{Q}$. et al. Impact of Surface Polyethylene Glycol (PEG) Density on Biodegradable Nanoparticle Transport in Mucus ex Vivo and Distribution in Vivo. ACS Nano 9, 9217-9227, doi:10.1021/acsnano.5b03876 (2015).

35 Yang, M. et al. Biodegradable nanoparticles composed entirely of safe materials that rapidly penetrate human mucus. Angew Chem Int Ed Engl 50, 2597-2600, doi:10.1002/anie.201006849 (2011).

36 Yang, M. et al. Vaginal delivery of paclitaxel via nanoparticles with non-mucoadhesive surfaces suppresses cervical tumor growth. Adv Healthc Mater 3, 1044-1052, doi:10.1002/adhm.201300519 (2014). 
$\mathrm{Yu}$, T. et al. Biodegradable mucus-penetrating nanoparticles composed of diblock copolymers of polyethylene glycol and poly(lactic-co-glycolic acid). Drug Deliv Transl Res 2, doi:10.1007/s13346-011-0048-9 (2012). das Neves, J., Amiji, M. \& Sarmento, B. Mucoadhesive nanosystems for vaginal microbicide development: friend or foe? Wiley Interdiscip Rev Nanomed Nanobiotechnol 3, 389-399, doi:10.1002/wnan.144 (2011).

Martin, D. T. et al. Surface-modified nanoparticles enhance transurothelial penetration and delivery of survivin siRNA in treating bladder cancer. Mol Cancer Ther 13, 71-81, doi:10.1158/1535-7163.MCT-13-0502 (2014).

40 Mert, O. et al. A poly(ethylene glycol)-based surfactant for formulation of drug-loaded mucus penetrating particles. J Control Release 157, 455-460, doi:10.1016/j.jconrel.2011.08.032 (2012).

41 Pereira, M. N. et al. Novel ex vivo protocol using porcine vagina to assess drug permeation from mucoadhesive and colloidal pharmaceutical systems. Colloids Surf B Biointerfaces 158, 222-228, doi:10.1016/j.colsurfb.2017.07.008 (2017).

$42 \mathrm{Cu}$, Y. \& Saltzman, W. M. Mathematical modeling of molecular diffusion through mucus. Adv Drug Deliv Rev 61, 101-114, doi:10.1016/j.addr.2008.09.006 (2009).

43 Olmsted, S. S. et al. Diffusion of macromolecules and virus-like particles in human cervical mucus. Biophys J 81, 1930-1937, doi:10.1016/S0006-3495(01)75844-4 (2001).

44 Boukari, H. et al. Movements of HIV-virions in human cervical mucus. Biomacromolecules 10, 2482-2488, doi:10.1021/bm900344q (2009).

45 Chen, A. et al. Modeling of Virion Collisions in Cervicovaginal Mucus Reveals Limits on Agglutination as the Protective Mechanism of Secretory Immunoglobulin A. PLoS One 10, e0131351, doi:10.1371/journal.pone.0131351 (2015).

46 Frieboes, H. B., Sinek, J. P., Nalcioglu, O., Fruehauf, J. P. \& Cristini, V. in BioMEMS and Biomedical Nanotechnology (ed Springer) (Springer, 2006).

47 Curtis, L. T., Rychahou, P., Bae, Y. \& Frieboes, H. B. A Computational/Experimental Assessment of Antitumor Activity of Polymer Nanoassemblies for $\mathrm{pH}$-Controlled Drug Delivery to Primary and Metastatic Tumors. Pharm Res 33, 2552-2564, doi:10.1007/s11095-016-1981-6 (2016).

48 Curtis, L. T., Wu, M., Lowengrub, J., Decuzzi, P. \& Frieboes, H. B. Computational Modeling of Tumor Response to Drug Release from Vasculature-Bound Nanoparticles. PLoS One 10, e0144888, doi:10.1371/journal.pone.0144888 (2015).

49 Decuzzi, P., Pasqualini, R., Arap, W. \& Ferrari, M. Intravascular delivery of particulate systems: does geometry really matter? Pharm Res 26, 235-243, doi:10.1007/s11095-008-9697-x (2009).

50 England, C. G., Gobin, A. M. \& Frieboes, H. B. Evaluation of uptake and distribution of gold nanoparticles in solid tumors. Eur Phys J Plus 130, doi:10.1140/epjp/i2015-15231-1 (2015).

51 Frieboes, H. B., Wu, M., Lowengrub, J., Decuzzi, P. \& Cristini, V. A computational model for predicting nanoparticle accumulation in tumor vasculature. PLoS One 8, e56876, doi:10.1371/journal.pone.0056876 (2013).

52 Gao, Y. et al. Predictive models of diffusive nanoparticle transport in 3-dimensional tumor cell spheroids. AAPS J 15, 816-831, doi:10.1208/s12248-013-9478-2 (2013).

53 Leonard, F. et al. Macrophage Polarization Contributes to the Anti-Tumoral Efficacy of Mesoporous Nanovectors Loaded with Albumin-Bound Paclitaxel. Front Immunol 8, 693, doi:10.3389/fimmu.2017.00693 (2017).

54 Leonard, F. et al. Enhanced performance of macrophage-encapsulated nanoparticle albuminbound-paclitaxel in hypo-perfused cancer lesions. Nanoscale 8, 12544-12552, doi:10.1039/c5nr07796f (2016).

55 Koren, E. \& Torchilin, V. P. Cell-penetrating peptides: breaking through to the other side. Trends in molecular medicine 18, 385-393, doi:10.1016/j.molmed.2012.04.012 (2012). 
Li, M. \& Reineke, J. Mathematical modelling of nanoparticle biodistribution: extrapolation among intravenous, oral and pulmonary administration routes. International Journal of Nano and Biomaterials 3, doi:10.1504/IJNBM.2011.042131 (2011).

Reichel, D. et al. Development of Halofluorochromic Polymer Nanoassemblies for the Potential Detection of Liver Metastatic Colorectal Cancer Tumors Using Experimental and Computational Approaches. Pharm Res 34, 2385-2402, doi:10.1007/s11095-017-2245-9 (2017).

Sinek, J., Frieboes, H., Zheng, X. \& Cristini, V. Two-dimensional chemotherapy simulations demonstrate fundamental transport and tumor response limitations involving nanoparticles. Biomed Microdevices 6, 297-309, doi:10.1023/B:BMMD.0000048562.29657.64 (2004). van de Ven, A. L. et al. Modeling of nanotherapeutics delivery based on tumor perfusion. New J Phys 15, 55004, doi:10.1088/1367-2630/15/5/055004 (2013). van de Ven, A. L. et al. Integrated intravital microscopy and mathematical modeling to optimize nanotherapeutics delivery to tumors. AIP Adv 2, 11208, doi:10.1063/1.3699060 (2012). Wu, M. et al. The effect of interstitial pressure on therapeutic agent transport: coupling with the tumor blood and lymphatic vascular systems. J Theor Biol 355, 194-207, doi:10.1016/j.jtbi.2014.04.012 (2014).

62 Curtis, L. T., England, C. G., Wu, M., Lowengrub, J. \& Frieboes, H. B. An interdisciplinary computational/experimental approach to evaluate drug-loaded gold nanoparticle tumor cytotoxicity. Nanomedicine-Uk 11, 197-216, doi:10.2217/nnm.15.195 (2016).

63 Curtis, L. T. \& Frieboes, H. B. The Tumor Microenvironment as a Barrier to Cancer Nanotherapy. Adv Exp Med Biol 936, 165-190, doi:10.1007/978-3-319-42023-3_9 (2016).

64 Godin, B. et al. An Integrated Approach for the Rational Design of Nanovectors for Biomedical Imaging and Therapy. Adv Genet 69, 31-64, doi:10.1016/S0065-2660(10)69009-8 (2010). Kaddi, C. D., Phan, J. H. \& Wang, M. D. Computational nanomedicine: modeling of nanoparticlemediated hyperthermal cancer therapy. Nanomedicine-Uk 8, 1323-1333, doi:10.2217/Nnm.13.117 (2013).

66 Li, M. G., Czyszczon, E. A. \& Reineke, J. J. Delineating intracellular pharmacokinetics of paclitaxel delivered by PLGA nanoparticles. Drug Deliv Transl Re 3, 551-561, doi:10.1007/s13346-0130162-y (2013).

67 Li, M. G., Panagi, Z., Avgoustakis, K. \& Reineke, J. Physiologically based pharmacokinetic modeling of PLGA nanoparticles with varied mPEG content. Int J Nanomed 7, 1345-1356, doi:10.2147/ljn.S23758 (2012).

68 Lu, M. G., Al-Jamal, K. T., Kostarelos, K. \& Reineke, J. Physiologically Based Pharmacokinetic Modeling of Nanoparticles. Acs Nano 4, 6303-6317, doi:10.1021/nn1018818 (2010).

69 Lorton, S. P., Kummerfeld, H. L. \& Foote, R. H. Polyacrylamide as a substitute for cervical mucus in sperm migration tests. Fertil Steril 35, 222-225 (1981).

70 Lai, S. K., Wang, Y. Y., Cone, R., Wirtz, D. \& Hanes, J. Altering mucus rheology to "solidify" human mucus at the nanoscale. PLoS One 4, e4294, doi:10.1371/journal.pone.0004294 (2009).

71 Saltzman, W. M., Radomsky, M. L., Whaley, K. J. \& Cone, R. A. Antibody diffusion in human cervical mucus. Biophys J 66, 508-515 (1994).

72 Willits, R. K. \& Saltzman, W. M. Synthetic polymers alter the structure of cervical mucus. Biomaterials 22, 445-452 (2001).

73 Gardner, J. K. \& Herbst-Kralovetz, M. M. Three-Dimensional Rotating Wall Vessel-Derived Cell Culture Models for Studying Virus-Host Interactions. Viruses 8, doi:10.3390/v8110304 (2016).

74 He, B., Chen, G. \& Zeng, Y. Three-dimensional cell culture models for investigating human viruses. Virol Sin 31, 363-379, doi:10.1007/s12250-016-3889-z (2016).

75 Le, T. T. D. et al. Evaluation of anti-HER2 scFv-conjugated PLGA-PEG nanoparticles on 3D tumor spheroids of BT474 and HCT116 cancer cells. Adv Nat Sci-Nanosci 7, doi:Artn 025004 
10.1088/2043-6262/7/2/025004 (2016).

76 Sobel, J. D., Tchao, R., Bozzola, J., Levison, M. E. \& Kaye, D. Adherence of Bacteria to Human Vaginal Epithelial-Cell Multilayer Tissue-Cultures. Clin Res 27, A481-A481 (1979).

77 van Eyk, A. D. \& van der Bijl, P. The culture of human buccal and vaginal epithelial cells for permeability studies. SADJ 53, 497-503 (1998).

78 Ensign, L. M. et al. Ex vivo characterization of particle transport in mucus secretions coating freshly excised mucosal tissues. Mol Pharm 10, 2176-2182, doi:10.1021/mp400087y (2013).

79 Yang, M. et al. Nanoparticle penetration of human cervicovaginal mucus: the effect of polyvinyl alcohol. J Control Release 192, 202-208, doi:10.1016/j.jconrel.2014.07.045 (2014).

80 Amsden, B. An obstruction-scaling model for diffusion in homogeneous hydrogels. Macromolecules 32, 874-879, doi:DOI 10.1021/ma980922a (1999).

81 Wessler, T. et al. Using Computational Modeling To Optimize the Design of Antibodies That Trap Viruses in Mucus. ACS Infect Dis 2, 82-92, doi:10.1021/acsinfecdis.5b00108 (2016).

82 Katz, D. F., Gao, Y. J. \& Chang, S. Multi-Compartmental Transport of Anti-HIV Molecules into and through Mucosa. Biophysical Journal 102, 594a-594a (2012).

83 Shen, H., Hu, Y. \& Saltzman, W. M. DNA diffusion in mucus: effect of size, topology of DNAs, and transfection reagents. Biophys J 91, 639-644, doi:10.1529/biophysj.105.077404 (2006).

84 Pyles, R. B. et al. Cultivated vaginal microbiomes alter HIV-1 infection and antiretroviral efficacy in colonized epithelial multilayer cultures. PLoS One 9, e93419, doi:10.1371/journal.pone.0093419 (2014).

85 Sobel, J. D., Tchao, R., Bozzola, J., Levison, M. E. \& Kaye, D. Human Vaginal Epithelial Multilayer Tissue-Culture. In Vitro Cell Dev B 15, 993-1000 (1979).

86 van Eyk, A. D. \& van der Bijl, P. Porcine vaginal mucosa as an in vitro permeability model for human vaginal mucosa. Int J Pharm 305, 105-111, doi:10.1016/j.ijpharm.2005.09.002 (2005).

87 Meng, J., Sturgis, T. F. \& Youan, B. B. Engineering tenofovir loaded chitosan nanoparticles to maximize microbicide mucoadhesion. Eur J Pharm Sci 44, 57-67, doi:10.1016/j.ejps.2011.06.007 (2011).

88 Ensign, L. M., Hoen, T. E., Maisel, K., Cone, R. A. \& Hanes, J. S. Enhanced vaginal drug delivery through the use of hypotonic formulations that induce fluid uptake. Biomaterials 34, 6922-6929, doi:10.1016/j.biomaterials.2013.05.039 (2013).

89 Geonnotti, A. R. \& Katz, D. F. Compartmental transport model of microbicide delivery by an intravaginal ring. J Pharm Sci 99, 3514-3521, doi:10.1002/jps.22120 (2010).

90 Jarrett, A. M., Gao, Y., Hussaini, M. Y., Cogan, N. G. \& Katz, D. F. Sensitivity Analysis of a Pharmacokinetic Model of Vaginal Anti-HIV Microbicide Drug Delivery. J Pharm Sci 105, 17721778, doi:10.1016/j.xphs.2016.02.015 (2016).

91 Gao, Y. et al. Vaginal deployment and tenofovir delivery by microbicide gels. Drug Deliv Transl Res 5, 279-294, doi:10.1007/s13346-015-0227-1 (2015).

92 Casalini, T., Rossi, F., Lazzari, S., Perale, G. \& Masi, M. Mathematical modeling of PLGA microparticles: from polymer degradation to drug release. Mol Pharm 11, 4036-4048, doi:10.1021/mp500078u (2014).

93 Halwes, M. E., Tyo, K. T., Steinbach-Rankins, J. M. \& Frieboes, H. B. Computational Modeling of Drug Diffusion from PLGA Fibers and Multicompartment Pharmacokinetics for Application to the Female Reproductive Tract. . Mol Pharm (In Press).

94 ACS. Learn About Cancer - Cervical Cancer - Detailed Guide - What are the key statistics about cervical cancer?, <http://www.cancer.org/cancer/cervicalcancer/detailedguide/cervical-cancerkey-statistics> (2016). 
Siegel, R., Ward, E., Brawley, O. \& Jemal, A. Cancer statistics, 2011: the impact of eliminating socioeconomic and racial disparities on premature cancer deaths. CA: a cancer journal for clinicians 61, 212-236, doi:10.3322/caac.20121 (2011).

96 CDC. Gynecologic Cancers, <http://www.cdc.gov/cancer/cervical/> (2015).

$97 \mathrm{NCl}$. NCl: HPV and Cancer, <http://www.cancer.gov/about-cancer/causesprevention/risk/infectious-agents/hpv-fact-sheet> (2015).

98 Schiller, J. T., Castellsague, X. \& Garland, S. M. A Review of Clinical Trials of Human Papillomavirus Prophylactic Vaccines. Vaccine 30, F123-F138, doi:10.1016/j.vaccine.2012.04.108 (2012).

99 Hildesheim, A. et al. Effect of human papillomavirus 16/18 L1 viruslike particle vaccine among young women with preexisting infection - A randomized trial. Jama-J Am Med Assoc 298, 743753, doi:DOI 10.1001/jama.298.7.743 (2007).

100 Chen, J. Z. et al. Nanotechnology in the management of cervical cancer. Rev Med Virol 25, 72-83, doi:10.1002/rmv.1825 (2015).

101 Jensen, P. T. et al. Early-stage cervical carcinoma, radical hysterectomy, and sexual function. A longitudinal study. Cancer 100, 97-106, doi:10.1002/cncr.11877 (2004).

102 Kyrgiou, M. et al. Obstetric outcomes after conservative treatment for intraepithelial or early invasive cervical lesions: systematic review and meta-analysis. Lancet 367, 489-498, doi:10.1016/S0140-6736(06)68181-6 (2006).

103 Bertrand, N., Wu, J., Xu, X., Kamaly, N. \& Farokhzad, O. C. Cancer nanotechnology: the impact of passive and active targeting in the era of modern cancer biology. Adv Drug Deliv Rev 66, 2-25, doi:10.1016/j.addr.2013.11.009 (2014).

104 Danhier, F. et al. PLGA-based nanoparticles: an overview of biomedical applications. J Control Release 161, 505-522, doi:10.1016/j.jconrel.2012.01.043 (2012).

105 Soppimath, K. S., Aminabhavi, T. M., Kulkarni, A. R. \& Rudzinski, W. E. Biodegradable polymeric nanoparticles as drug delivery devices. J Control Release 70, 1-20 (2001).

106 Dawidczyk, C. M. et al. State-of-the-art in design rules for drug delivery platforms: lessons learned from FDA-approved nanomedicines. J Control Release 187, 133-144, doi:10.1016/j.jconrel.2014.05.036 (2014).

107 Salmaso, S. \& Caliceti, P. Stealth properties to improve therapeutic efficacy of drug nanocarriers. Journal of drug delivery 2013, 374252, doi:10.1155/2013/374252 (2013).

108 Amoozgar, Z. \& Yeo, Y. Recent advances in stealth coating of nanoparticle drug delivery systems. Wiley Interdiscip Rev Nanomed Nanobiotechnol 4, 219-233, doi:10.1002/wnan.1157 (2012).

109 Alexander-Bryant, A. A., Vanden Berg-Foels, W. S. \& Wen, X. Bioengineering strategies for designing targeted cancer therapies. Advances in cancer research 118, 1-59, doi:10.1016/B9780-12-407173-5.00002-9 (2013).

110 Erazo-Oliveras, A., Muthukrishnan, N., Baker, R., Wang, T. Y. \& Pellois, J. P. Improving the endosomal escape of cell-penetrating peptides and their cargos: strategies and challenges. Pharmaceuticals (Basel) 5, 1177-1209, doi:10.3390/ph5111177 (2012).

111 Lehto, T., Kurrikoff, K. \& Langel, U. Cell-penetrating peptides for the delivery of nucleic acids. Expert Opin Drug Deliv 9, 823-836, doi:10.1517/17425247.2012.689285 (2012).

112 Margus, H., Padari, K. \& Pooga, M. Cell-penetrating peptides as versatile vehicles for oligonucleotide delivery. Molecular therapy : the journal of the American Society of Gene Therapy 20, 525-533, doi:10.1038/mt.2011.284 (2012).

113 Meade, B. R. \& Dowdy, S. F. Exogenous siRNA delivery using peptide transduction domains/cell penetrating peptides. Adv Drug Deliv Rev 59, 134-140, doi:10.1016/j.addr.2007.03.004 (2007). 
114 Meade, B. R. \& Dowdy, S. F. Enhancing the cellular uptake of siRNA duplexes following noncovalent packaging with protein transduction domain peptides. Adv Drug Deliv Rev 60, 530536 (2008).

115 Morris, M. C., Deshayes, S., Heitz, F. \& Divita, G. Cell-penetrating peptides: from molecular mechanisms to therapeutics. Biology of the cell / under the auspices of the European Cell Biology Organization 100, 201-217, doi:10.1042/BC20070116 (2008).

116 Mussbach, F., Franke, M., Zoch, A., Schaefer, B. \& Reissmann, S. Transduction of peptides and proteins into live cells by cell penetrating peptides. Journal of cellular biochemistry 112, 38243833, doi:10.1002/jcb.23313 (2011).

117 Henriques, S. T., Melo, M. N. \& Castanho, M. A. Cell-penetrating peptides and antimicrobial peptides: how different are they? The Biochemical journal 399, 1-7, doi:10.1042/BJ20061100 (2006).

118 Raucher, D. \& Ryu, J. S. Cell-penetrating peptides: strategies for anticancer treatment. Trends in molecular medicine 21, 560-570, doi:10.1016/j.molmed.2015.06.005 (2015).

119 Gupta, B., Levchenko, T. S. \& Torchilin, V. P. Intracellular delivery of large molecules and small particles by cell-penetrating proteins and peptides. Adv Drug Deliv Rev 57, 637-651, doi:10.1016/j.addr.2004.10.007 (2005).

120 Mokhtarieh, A. A., Kim, S., Lee, Y., Chung, B. H. \& Lee, M. K. Novel cell penetrating peptides with multiple motifs composed of RGD and its analogs. Biochemical and biophysical research communications 432, 359-364, doi:10.1016/j.bbrc.2013.01.096 (2013).

121 Simeoni, F., Morris, M. C., Heitz, F. \& Divita, G. Insight into the mechanism of the peptide-based gene delivery system MPG: implications for delivery of siRNA into mammalian cells. Nucleic acids research 31, 2717-2724 (2003).

122 Minchinton, A. I. \& Tannock, I. F. Drug penetration in solid tumours. Nature reviews. Cancer 6, 583-592, doi:10.1038/nrc1893 (2006).

123 Sutherland, R., McCredie, J. \& Inch, W. Growth of multicell spheroids in tissue culture as a model of nodular carcinomas. J Natl Cancer Inst 46, 113 - 120 (1971).

124 Curtis, L. T., England, C. G., Wu, M., Lowengrub, J. \& Frieboes, H. B. An interdisciplinary computational/experimental approach to evaluate drug-loaded gold nanoparticle tumor cytotoxicity. Nanomedicine (Lond) 11, 197-216, doi:10.2217/nnm.15.195 (2016).

125 Goodman, T. T., Olive, P. L. \& Pun, S. H. Increased nanoparticle penetration in collagenasetreated multicellular spheroids. Int J Nanomedicine 2, 265-274 (2007).

126 Huang, K. et al. Size-dependent localization and penetration of ultrasmall gold nanoparticles in cancer cells, multicellular spheroids, and tumors in vivo. ACS Nano 6, 4483-4493, doi:10.1021/nn301282m (2012).

127 Neshatian, M., Chung, S., Yohan, D., Yang, C. \& Chithrani, D. B. Uptake of Gold Nanoparticles in Breathless (Hypoxic) Cancer Cells. Journal of biomedical nanotechnology 11, 1162-1172 (2015).

128 Li, M., Panagi, Z., Avgoustakis, K. \& Reineke, J. Physiologically based pharmacokinetic modeling of PLGA nanoparticles with varied mPEG content. Int J Nanomedicine 7, 1345-1356, doi:10.2147/IJN.S23758 (2012).

129 Decuzzi, P. et al. Size and shape effects in the biodistribution of intravascularly injected particles. J Control Release 141, 320-327, doi:10.1016/j.jconrel.2009.10.014 (2010).

130 Boso, D. P., Lee, S. Y., Ferrari, M., Schrefler, B. A. \& Decuzzi, P. Optimizing particle size for targeting diseased microvasculature: from experiments to artificial neural networks. Int J Nanomedicine 6, 1517-1526, doi:10.2147/IJN.S20283 (2011).

131 Decuzzi, P. \& Ferrari, M. The adhesive strength of non-spherical particles mediated by specific interactions. Biomaterials 27, 5307-5314, doi:10.1016/j.biomaterials.2006.05.024 (2006). 
132 Lee, S. Y., Ferrari, M. \& Decuzzi, P. Shaping nano-/micro-particles for enhanced vascular interaction in laminar flows. Nanotechnology 20, 495101, doi:10.1088/09574484/20/49/495101 (2009).

133 Curtis, L. T., Wu, M., Lowengrub, J., Decuzzi, P. \& Frieboes, H. B. Computational Modeling of Tumor Response to Drug Release from Vasculature-Bound Nanoparticles. PloS one in press (2015).

134 Balzeau, J., Peterson, A. \& Eyer, J. The vimentin-tubulin binding site peptide (Vim-TBS.58-81) crosses the plasma membrane and enters the nuclei of human glioma cells. Int J Pharmaceut 423, 77-83, doi:10.1016/j.ijpharm.2011.04.067 (2012).

135 Saini, A., Jaswal, R. R., Negi, R. \& Nandel, F. S. Insights on the structural characteristics of VimTBS (58-81) peptide for future applications as a cell penetrating peptide. Biosci Trends 7, 209220, doi:10.5582/bst.2013.v7.5.209 (2013).

136 Fahmy, T. M., Samstein, R. M., Harness, C. C. \& Mark Saltzman, W. Surface modification of biodegradable polyesters with fatty acid conjugates for improved drug targeting. Biomaterials 26, 5727-5736, doi:10.1016/j.biomaterials.2005.02.025 (2005).

$137 \mathrm{Cu}, \mathrm{Y} ., \mathrm{Booth}, \mathrm{C}$. J. \& Saltzman, W. M. In vivo distribution of surface-modified PLGA nanoparticles following intravaginal delivery. J Control Release 156, 258-264, doi:10.1016/j.jconrel.2011.06.036 (2011).

$138 \mathrm{Li}$, J. et al. Targeting the brain with PEG-PLGA nanoparticles modified with phage-displayed peptides. Biomaterials 32, 4943-4950, doi:10.1016/j.biomaterials.2011.03.031 (2011).

139 Desai, M. P., Labhasetwar, V., Walter, E., Levy, R. J. \& Amidon, G. L. The mechanism of uptake of biodegradable microparticles in Caco-2 cells is size dependent. Pharm Res 14, 1568-1573 (1997).

140 Polacheck, W. J., Zervantonakis, I. K. \& Kamm, R. D. Tumor cell migration in complex microenvironments. Cell Mol Life Sci 70, 1335-1356, doi:10.1007/s00018-012-1115-1 (2013).

141 Tchafa, A. M., Shah, A. D., Wang, S., Duong, M. T. \& Shieh, A. C. Three-dimensional cell culture model for measuring the effects of interstitial fluid flow on tumor cell invasion. Journal of visualized experiments : JoVE, doi:10.3791/4159 (2012).

142 Yamada, K. M. \& Cukierman, E. Modeling tissue morphogenesis and cancer in 3D. Cell 130, 601610, doi:10.1016/j.cell.2007.08.006 (2007).

143 Griffith, L. G. \& Swartz, M. A. Capturing complex 3D tissue physiology in vitro. Nature reviews. Molecular cell biology 7, 211-224, doi:10.1038/nrm1858 (2006).

144 Lee, J., Lilly, G. D., Doty, R. C., Podsiadlo, P. \& Kotov, N. A. In vitro toxicity testing of nanoparticles in 3D cell culture. SMALL 5, 1213-1221, doi:10.1002/smll.200801788 (2009).

145 Nyga, A., Cheema, U. \& Loizidou, M. 3D tumour models: novel in vitro approaches to cancer studies. Journal of cell communication and signaling 5, 239-248, doi:10.1007/s12079-011-0132-4 (2011).

146 Santini, M. T., Rainaldi, G. \& Indovina, P. L. Apoptosis, cell adhesion and the extracellular matrix in the three-dimensional growth of multicellular tumor spheroids. Critical reviews in oncology/hematology 36, 75-87 (2000).

147 Roberts, W. G. \& Palade, G. E. Increased microvascular permeability and endothelial fenestration induced by vascular endothelial growth factor. Journal of cell science 108 ( Pt 6), 2369-2379 (1995).

148 Primeau, A. J., Rendon, A., Hedley, D., Lilge, L. \& Tannock, I. F. The distribution of the anticancer drug Doxorubicin in relation to blood vessels in solid tumors. Clinical cancer research : an official journal of the American Association for Cancer Research 11, 8782-8788, doi:10.1158/10780432.CCR-05-1664 (2005).

149 Hait, W. N. \& Hambley, T. W. Targeted cancer therapeutics. Cancer research 69, 1263-1267; discussion 1267, doi:10.1158/0008-5472.CAN-08-3836 (2009). 
150 Warren, K. E. Novel therapeutic delivery approaches in development for pediatric gliomas. CNS oncology 2, 427-435, doi:10.2217/cns.13.37 (2013).

151 England, C. G. et al. Enhanced penetration into 3D cell culture using two and three layered gold nanoparticles. Int J Nanomed 8, 3602-3617 (2013).

152 England, C. G. et al. Detection of Phosphatidylcholine-Coated Gold Nanoparticles in Orthotopic Pancreatic Adenocarcinoma using Hyperspectral Imaging. PloS one 10, e0129172, doi:10.1371/journal.pone.0129172 (2015).

153 England, C. G., Miller, M. C., Kuttan, A., Trent, J. O. \& Frieboes, H. B. Release kinetics of paclitaxel and cisplatin from two and three layered gold nanoparticles. European journal of pharmaceutics and biopharmaceutics : official journal of Arbeitsgemeinschaft fur Pharmazeutische Verfahrenstechnik e.V 92, 120-129, doi:10.1016/j.ejpb.2015.02.017 (2015).

154 Dissanayake, S., Denny, W. A., Gamage, S. \& Sarojini, V. Recent developments in anticancer drug delivery using cell penetrating and tumor targeting peptides. J Control Release 250, 62-76, doi:10.1016/j.jconrel.2017.02.006 (2017).

155 Farkhani, S. M. et al. Cell penetrating peptides: efficient vectors for delivery of nanoparticles, nanocarriers, therapeutic and diagnostic molecules. Peptides 57, 78-94, doi:10.1016/j.peptides.2014.04.015 (2014).

156 Kamaly, N., Xiao, Z., Valencia, P. M., Radovic-Moreno, A. F. \& Farokhzad, O. C. Targeted polymeric therapeutic nanoparticles: design, development and clinical translation. Chem Soc Rev 41, 2971-3010, doi:10.1039/c2cs15344k (2012).

157 Ordikhani, F. et al. Drug Delivery Approaches for the Treatment of Cervical Cancer. Pharmaceutics 8, doi:10.3390/pharmaceutics8030023 (2016).

158 Shin, M. C. et al. Cell-penetrating peptides: achievements and challenges in application for cancer treatment. J Biomed Mater Res A 102, 575-587, doi:10.1002/jbm.a.34859 (2014).

159 Eetezadi, S., De Souza, R., Vythilingam, M., Lessa Cataldi, R. \& Allen, C. Effects of Doxorubicin Delivery Systems and Mild Hyperthermia on Tissue Penetration in 3D Cell Culture Models of Ovarian Cancer Residual Disease. Mol Pharm 12, 3973-3985, doi:10.1021/acs.molpharmaceut.5b00426 (2015).

160 Gao, W., Xiang, B., Meng, T. T., Liu, F. \& Qi, X. R. Chemotherapeutic drug delivery to cancer cells using a combination of folate targeting and tumor microenvironment-sensitive polypeptides. Biomaterials 34, 4137-4149, doi:10.1016/j.biomaterials.2013.02.014 (2013).

161 Zhang, C., Zhang, Z. \& Zhao, L. Folate-decorated poly(3-hydroxybutyrate-co-3hydroxyoctanoate) nanoparticles for targeting delivery: optimization and in vivo antitumor activity. Drug Deliv 23, 1830-1837, doi:10.3109/10717544.2015.1122675 (2016).

162 Huang, S. et al. Tumor targeting and microenvironment-responsive nanoparticles for gene delivery. Biomaterials 34, 5294-5302, doi:10.1016/j.biomaterials.2013.03.043 (2013).

$163 \mathrm{Ma}, \mathrm{Y}$. D. et al. Nanoparticle formulation of poly(epsilon-caprolactone-co-lactide)-D-alphatocopheryl polyethylene glycol 1000 succinate random copolymer for cervical cancer treatment. Polymer 51, 5952-5959, doi:10.1016/j.polymer.2010.10.029 (2010).

164 Yang, H., Li, K., Liu, Y., Liu, Z. \& Miyoshi, H. Poly(D,L-lactide-co-glycolide) nanoparticles encapsulated fluorescent isothiocyanate and paclitaxol: preparation, release kinetics and anticancer effect. J Nanosci Nanotechnol 9, 282-287 (2009).

165 Qiu, B. et al. Co-delivery of docetaxel and endostatin by a biodegradable nanoparticle for the synergistic treatment of cervical cancer. Nanoscale Res Lett 7, 666, doi:10.1186/1556-276X-7666 (2012).

166 Krishnakumar, N. S., N.; RajendraPrasad, N.; Karthikeyan, S. Enhanced anticancer activity of naringenin-loaded nanoparticles in human cervical (HeLa) cancer cells. Biomed. Prev. Nutr 1, 9 (2011). 
167 Tang, H. et al. Stability, Pharmacokinetics, Biodistribution and Safety Assessment of FolateConjugated Pullulan Acetate Nanoparticles as Cervical Cancer Targeted Drug Carriers. J Nanosci Nanotechnol 15, 6405-6412 (2015).

168 Peng, W. et al. DNA nanotherapy for pre-neoplastic cervical lesions. Gynecol Oncol 128, 101106, doi:10.1016/j.ygyno.2012.10.018 (2013).

169 Blum, J. S. et al. Prevention of K-Ras- and Pten-mediated intravaginal tumors by treatment with camptothecin-loaded PLGA nanoparticles. Drug Deliv Transl Res 1, 383-394, doi:10.1007/s13346-011-0038-y (2011).

170 Raghavan, S. et al. Comparative analysis of tumor spheroid generation techniques for differential in vitro drug toxicity. Oncotarget 7, 16948-16961, doi:10.18632/oncotarget.7659 (2016).

171 Zhang, P., Cheetham, A. G., Lock, L. L. \& Cui, H. Cellular uptake and cytotoxicity of drug-peptide conjugates regulated by conjugation site. Bioconjug Chem 24, 604-613, doi:10.1021/bc300585h (2013).

172 Morris, M. C., Vidal, P., Chaloin, L., Heitz, F. \& Divita, G. A new peptide vector for efficient delivery of oligonucleotides into mammalian cells. Nucleic acids research 25, 2730-2736 (1997).

173 Gao, H. et al. Ligand modified nanoparticles increases cell uptake, alters endocytosis and elevates glioma distribution and internalization. Sci Rep 3, 2534, doi:10.1038/srep02534 (2013).

174 Lee, H., Fonge, H., Hoang, B., Reilly, R. M. \& Allen, C. The effects of particle size and molecular targeting on the intratumoral and subcellular distribution of polymeric nanoparticles. Mol Pharm 7, 1195-1208, doi:10.1021/mp100038h (2010).

175 Mikhail, A. S., Eetezadi, S. \& Allen, C. Multicellular tumor spheroids for evaluation of cytotoxicity and tumor growth inhibitory effects of nanomedicines in vitro: a comparison of docetaxelloaded block copolymer micelles and Taxotere(R). PLoS One 8, e62630, doi:10.1371/journal.pone.0062630 (2013).

176 Lee, J. J., Lee, S. Y., Park, J. H., Kim, D. D. \& Cho, H. J. Cholesterol-modified poly(lactide-coglycolide) nanoparticles for tumor-targeted drug delivery. Int J Pharm 509, 483-491, doi:10.1016/j.ijpharm.2016.06.008 (2016).

177 Goodman, T. T., Chen, J., Matveev, K. \& Pun, S. H. Spatio-temporal modeling of nanoparticle delivery to multicellular tumor spheroids. Biotechnol Bioeng 101, 388-399, doi:10.1002/bit.21910 (2008).

178 Lee, H., Hoang, B., Fonge, H., Reilly, R. M. \& Allen, C. In vivo distribution of polymeric nanoparticles at the whole-body, tumor, and cellular levels. Pharm Res 27, 2343-2355, doi:10.1007/s11095-010-0068-z (2010).

179 Lee, S. Y. \& Cho, H. J. Amine-functionalized poly(lactic-co-glycolic acid) nanoparticles for improved cellular uptake and tumor penetration. Colloids Surf B Biointerfaces 148, 85-94, doi:10.1016/j.colsurfb.2016.08.050 (2016).

180 Froehlich, K. et al. Generation of Multicellular Breast Cancer Tumor Spheroids: Comparison of Different Protocols. J Mammary Gland Biol Neoplasia 21, 89-98, doi:10.1007/s10911-016-9359-2 (2016).

181 Frieboes, H. B. et al. Prediction of Drug Response in Breast Cancer Using Integrative Experimental/Computational Modeling. Cancer research 69, 4484-4492, doi:10.1158/00085472.can-08-37.10 (2009).

182 Frieboes, H. B. et al. An integrated computational/experimental model of tumor invasion. Cancer research 66, 1597-1604, doi:10.1158/0008-5472.can-05-3166 (2006).

183 Trédan, O., Galmarini, C. M., Patel, K. \& Tannock, I. F. Drug resistance and the solid tumor microenvironment. J. Natl. Cancer Inst. 99, 1441-1454, doi:10.1093/jnci/djm135 (2007). 
184 Herrmann, D. et al. Three-dimensional cancer models mimic cell-matrix interactions in the tumour microenvironment. Carcinogenesis 35, 1671-1679, doi:10.1093/carcin/bgu108 (2014).

185 Gorodeski, G. I., Jin, W. \& Hopfer, U. Extracellular Ca2+ directly regulates tight junctional permeability in the human cervical cell line CaSki. Am J Physiol 272, C511-524 (1997).

186 Siadat-Pajouh, M. et al. Detection of human papillomavirus type 16/18 DNA in cervicovaginal cells by fluorescence based in situ hybridization and automated image cytometry. Cytometry 15, 245-257, doi:10.1002/cyto.990150310 (1994).

187 Aasen, T., Hodgins, M. B., Edward, M. \& Graham, S. V. The relationship between connexins, gap junctions, tissue architecture and tumour invasion, as studied in a novel in vitro model of HPV16-associated cervical cancer progression. Oncogene 22, 7969-7980, doi:10.1038/sj.onc.1206709 (2003).

188 Lopez, J., Valdez-Morales, F. J., Benitez-Bribiesca, L., Cerbon, M. \& Carranca, A. G. Normal and cancer stem cells of the human female reproductive system. Reprod Biol Endocrin 11, doi:Artn 53

10.1186/1477-7827-11-53 (2013).

189 Globocan. Cervical Cancer Estimated Incidence, Mortality and Prevalence Worldwide in 2012, $<$ http://globocan.iarc.fr/old/FactSheets/cancers/cervix-new.asp> (2012).

190 About Cervical Cancer, <https://www.cancer.org/cancer/cervical-cancer/about/keystatistics.html> (2017).

191 International, W. C. R. F. Cervical Cancer Statistics <http://www.wcrf.org/int/cancer-factsfigures/data-specific-cancers/cervical-cancer-statistics> (2015).

192 Center, U. O. M. M. Cancer, <http://www.umm.edu/health/medical/reports/articles/cervical-cancer> (2012).

193 Institute, N. C. (ed SEER Cancer Statistics Review) (1975-2013).

194 Kang, Y. J. et al. Effect of changes in treatment practice on survival for cervical cancer: results from a population-based study in Manitoba, Canada. BMC Cancer 15, 642, doi:10.1186/s12885015-1624-z (2015).

195 Lorusso, D., Petrelli, F., Coinu, A., Raspagliesi, F. \& Barni, S. A systematic review comparing cisplatin and carboplatin plus paclitaxel-based chemotherapy for recurrent or metastatic cervical cancer. Gynecol Oncol 133, 117-123, doi:10.1016/j.ygyno.2014.01.042 (2014).

$196 \mathrm{Au}$, J. L., Jang, S. H. \& Wientjes, M. G. Clinical aspects of drug delivery to tumors. J Control Release 78, 81-95 (2002).

197 Cukierman, E. \& Khan, D. R. The benefits and challenges associated with the use of drug delivery systems in cancer therapy. Biochem Pharmacol 80, 762-770, doi:10.1016/j.bcp.2010.04.020 (2010).

198 Gupta, S. \& Gupta, M. K. Possible role of nanocarriers in drug delivery against cervical cancer. Nano Rev Exp 8, 1-25, doi:Artn 1335567

10.1080/20022727.2017.1335567 (2017).

199 Kijanka, M., Dorresteijn, B., Oliveira, S. \& van Bergen en Henegouwen, P. M. Nanobody-based cancer therapy of solid tumors. Nanomedicine (Lond) 10, 161-174, doi:10.2217/nnm.14.178 (2015).

200 Lee, B. K., Yun, Y. H. \& Park, K. Smart Nanoparticles for Drug Delivery: Boundaries and Opportunities. Chem Eng Sci 125, 158-164, doi:10.1016/j.ces.2014.06.042 (2015).

201 Parveen, S., Misra, R. \& Sahoo, S. K. Nanoparticles: a boon to drug delivery, therapeutics, diagnostics and imaging. Nanomedicine-Uk 8, 147-166, doi:10.1016/j.nano.2011.05.016 (2012). 
202 Zeng, X. et al. Cholic acid-functionalized nanoparticles of star-shaped PLGA-vitamin E TPGS copolymer for docetaxel delivery to cervical cancer. Biomaterials 34, 6058-6067, doi:10.1016/j.biomaterials.2013.04.052 (2013).

203 Grigore, M. E. Organic and Inorganic Nano-Systems Used in Cancer Treatment. Journal of Medical Research and Health Education 1 (2017).

204 Duman, F. D. et al. Folic acid-conjugated cationic Ag2S quantum dots for optical imaging and selective doxorubicin delivery to HeLa cells. Nanomedicine (Lond) 12, 2319-2333, doi:10.2217/nnm-2017-0180 (2017).

205 Zhitnyak, I. Y. et al. Effect of BN Nanoparticles Loaded with Doxorubicin on Tumor Cells with Multiple Drug Resistance. ACS Appl Mater Interfaces 9, 32498-32508, doi:10.1021/acsami.7b08713 (2017).

206 Wu, G. C., Wang, Z. Z., Bian, X. S., Du, X. J. \& Wei, C. H. Folate-modified doxorubicin-loaded nanoparticles for tumor-targeted therapy. Pharm Biol 52, 978-982, doi:10.3109/13880209.2013.874533 (2014).

207 De Jong, W. H. \& Borm, P. J. Drug delivery and nanoparticles:applications and hazards. Int J Nanomedicine 3, 133-149 (2008).

208 Durymanov, M. O., Rosenkranz, A. A. \& Sobolev, A. S. Current Approaches for Improving Intratumoral Accumulation and Distribution of Nanomedicines. Theranostics 5, 1007-1020, doi:10.7150/thno.11742 (2015).

209 de la Puente, P. et al. 3D tissue-engineered bone marrow as a novel model to study pathophysiology and drug resistance in multiple myeloma. Biomaterials 73, 70-84, doi:10.1016/j.biomaterials.2015.09.017 (2015).

210 Ordikhani, F., Kim, Y. \& Zustiak, S. P. The Role of Biomaterials on Cancer Stem Cell Enrichment and Behavior. Jom-Us 67, 2543-2549, doi:10.1007/s11837-015-1626-y (2015).

211 Beáta Tóth, P. K., Rémi Magnan. in Biomarkers in Toxicology (ed Academic Press) Ch. 56, 947963 (Academic Press, 2014).

212 Baek, N., Seo, O. W., Kim, M., Hulme, J. \& An, S. S. Monitoring the effects of doxorubicin on 3Dspheroid tumor cells in real-time. Onco Targets Ther 9, 7207-7218, doi:10.2147/OTT.S112566 (2016).

213 Grantab, R., Sivananthan, S. \& Tannock, I. F. The penetration of anticancer drugs through tumor tissue as a function of cellular adhesion and packing density of tumor cells. Cancer research 66, 1033-1039, doi:10.1158/0008-5472.CAN-05-3077 (2006).

214 Kuh, H. J., Jang, S. H., Wientjes, M. G., Weaver, J. R. \& Au, J. L. Determinants of paclitaxel penetration and accumulation in human solid tumor. J Pharmacol Exp Ther 290, 871-880 (1999).

215 Tredan, O., Galmarini, C. M., Patel, K. \& Tannock, I. F. Drug resistance and the solid tumor microenvironment. J Nat/ Cancer Inst 99, 1441-1454, doi:10.1093/jnci/djm135 (2007).

216 Lopez, J., Valdez-Morales, F. J., Benitez-Bribiesca, L., Cerbon, M. \& Carranca, A. G. Normal and cancer stem cells of the human female reproductive system. Reprod Biol Endocrinol 11, 53, doi:10.1186/1477-7827-11-53 (2013).

217 Hoppe-Seyler, K. et al. Viral E6/E7 oncogene and cellular hexokinase 2 expression in HPVpositive cancer cell lines. Oncotarget 8, 106342-106351, doi:10.18632/oncotarget.22463 (2017).

218 Hoppe-Seyler, K. et al. Induction of dormancy in hypoxic human papillomavirus-positive cancer cells. Proc Natl Acad Sci U S A 114, E990-E998, doi:10.1073/pnas.1615758114 (2017).

219 Comerford, K. M. et al. Hypoxia-inducible factor-1-dependent regulation of the multidrug resistance (MDR1) gene. Cancer research 62, 3387-3394 (2002).

220 Rice, G. C., Hoy, C. \& Schimke, R. T. Transient hypoxia enhances the frequency of dihydrofolate reductase gene amplification in Chinese hamster ovary cells. Proc Natl Acad Sci U S A 83, 59785982 (1986). 
221 Rice, G. C., Ling, V. \& Schimke, R. T. Frequencies of independent and simultaneous selection of Chinese hamster cells for methotrexate and doxorubicin (adriamycin) resistance. Proc Natl Acad Sci U S A 84, 9261-9264 (1987). 